\title{
Conflict Set and Waveform Modelling for Power Amplifier Design
}

\author{
Anamarija Juhas and Ladislav A. Novak \\ Department of Power, Electronics and Communication Engineering, Faculty of Technical Sciences, University of Novi Sad, \\ 21000 Novi Sad, Serbia \\ Correspondence should be addressed to Ladislav A. Novak; ladislav@uns.ac.rs
}

Received 19 July 2014; Accepted 7 February 2015

Academic Editor: Ben T. Nohara

Copyright (C) 2015 A. Juhas and L. A. Novak. This is an open access article distributed under the Creative Commons Attribution License, which permits unrestricted use, distribution, and reproduction in any medium, provided the original work is properly cited.

\begin{abstract}
Various classes of nonnegative waveforms containing dc component, fundamental and $k$ th harmonic $(k \geq 2)$, which proved to be of interest in waveform modelling for power amplifier (PA) design, are considered in this paper. In optimization of PA efficiency, nonnegative waveforms with maximal amplitude of fundamental harmonic and those with maximal coefficient of cosine term of fundamental harmonic (optimal waveforms) play an important role. Optimal waveforms have multiple global minima and this fact closely relates the problem of optimization of PA efficiency to the concept of conflict set. There is also keen interest in finding descriptions for various classes of suboptimal waveforms, such as nonnegative waveforms with at least one zero, nonnegative waveforms with maximal amplitude of fundamental harmonic for prescribed amplitude of $k$ th harmonic, nonnegative waveforms with maximal coefficient of cosine part of fundamental harmonic for prescribed coefficients of $k$ th harmonic, and nonnegative cosine waveforms with at least one zero. Closed form descriptions for all these suboptimal types of waveforms are provided in this paper. Suboptimal waveforms may also have multiple global minima and therefore be related to the concept of conflict set. Four case studies of usage of closed form descriptions of nonnegative waveforms in PA modelling are also provided.
\end{abstract}

\section{Introduction}

The origin of the concept of conflict set goes back to J. C. Maxwell (Maxwell 1831-1879), who informally introduced most of features of what today is called conflict set [1]. From this reason Maxwell set or Maxwell stratum is also used as synonyms for conflict set. Roughly speaking, conflict set associated with a smooth function $f$ with $m$ parameters is the set of $m$-tuples in parameter space for which $f$ has multiple global minima. Conflict set is also intimately related to singularity theory and catastrophe theory [1].

Although without explicit reference, many max-min/ min-max engineering design problems related to nonsmooth optimizations in parameter spaces (e.g., see [2]), including problems related to the optimization of efficiency of power amplifiers (PAs) (e.g., see [3-12]), are connected to the concept of conflict set. The concept of conflict set has been also used in mathematics (e.g., see [13-16]) and physics (e.g., see $[17,18])$, including subjects like black holes [19].
Nonnegative waveforms with maximal amplitude of fundamental harmonic and those with maximal coefficient of cosine term of fundamental harmonic (optimal waveforms) have multiple global minima and therefore are closely related to the concept of conflict set. The suboptimal waveforms such as

(i) nonnegative waveforms with at least one zero,

(ii) nonnegative waveforms with maximal amplitude of fundamental harmonic for prescribed amplitude of $k$ th harmonic,

(iii) nonnegative waveforms with maximal coefficient of cosine part of fundamental harmonic for prescribed coefficients of $k$ th harmonic,

(iv) nonnegative cosine waveforms with at least one zero

may also have multiple global minima $[9,11,12]$ and therefore be related to the concept of conflict set, as well. These suboptimal waveforms are clearly of interest in shaping/modelling 
drain (collector/plate) waveforms in PA design (e.g., see [3$12,20,21])$.

Fejér in his seminal paper [22] provided general description of all nonnegative trigonometric polynomials with $n$ consecutive harmonics in terms of $2 n+2$ parameters satisfying one nonlinear constraint. He also derived closed form solution to the problem of finding maximum possible amplitude of the first harmonic of nonnegative cosine polynomials with consecutive harmonics.

Fuzik [3] (see also [10]) considered cosine polynomials with $\mathrm{dc}$, fundamental and $k$ th harmonic, for arbitrary $k \geq 2$ and provided closed form solution for coefficients of optimal waveform. Rhodes in [7] provided closed form expression for maximum possible amplitude of fundamental harmonic of nonnegative waveforms containing consecutive odd harmonics. A subclass of nonnegative cosine waveforms with $\mathrm{dc}$, fundamental and third harmonic, having factorized form description has been considered in [23].

High efficiency PA with arbitrary output harmonic terminations has been analysed in [9], along with maximal efficiency, fundamental output power, and load impedance.

Factorized form of nonnegative waveforms up to second harmonic with at least one zero has been suggested in [11] in the context of continuous class B/J mode of PA operation.

General description of all nonnegative waveforms up to second harmonic in terms of four independent parameters has been provided in [12]. This includes nonnegative waveforms with at least one zero, as a special case.

End point of conflict set normally corresponds to socalled maximally flat waveform, which also belongs to class of suboptimal waveforms. First comprehensive usage of maximally flat waveforms, in the context of analysis of PA, goes to Raab [20]. General description of maximally flat waveforms with arbitrary number of harmonics has been presented in [21], along with closed form expressions for efficiency of class-F and inverse class-F PA with maximally flat waveforms. Description of maximally flat cosine waveforms with consecutive harmonics has been presented in [8] in the context of finite harmonic class-C PA.

In this paper we provide general descriptions of a number of optimal and suboptimal nonnegative waveforms containing dc component, fundamental and an arbitrary $k$ th harmonic, $k \geq 2$, and show how they are related to the concept of conflict set. According to our best knowledge, this paper provides the very first usage of conflict set in the course of solving problems related to optimization of PA efficiency. Main results are stated in six propositions (Propositions 1, 6, $9,18,22$, and 26), four corollaries (Corollaries 2-5), twenty remarks, and three algorithms. Four case studies of usage of closed form descriptions of nonnegative waveforms in PA efficiency analysis are considered in detail in Section 7.

This paper is organized in the following way. In Section 2 we introduce concepts of minimum function and gain function (Section 2.1), conflict set (Section 2.2), and parameter space (Section 2.3). In Sections 3-6 we provide general descriptions of various classes of nonnegative waveforms containing dc component, fundamental and $k$ th harmonic with at least one zero. General case of nonnegative waveforms with at least one zero is presented in Section 3.1. The case with exactly two zeros is considered in Section 3.2. An algorithm for calculation of coefficients of fundamental harmonic of nonnegative waveforms with two zeros, for prescribed coefficients of $k$ th harmonic, is presented in Section 3.3. Description of nonnegative waveforms with maximal amplitude of fundamental harmonic for prescribed amplitude of $k$ th harmonic is provided in Section 4. Nonnegative waveforms with maximal coefficient of cosine part of fundamental harmonic for prescribed coefficients of $k$ th harmonic are considered in Section 5.1. An illustration of results of Section 5.1 for particular case $k=3$ is given in Section 5.2. Section 6.1 is devoted to nonnegative cosine waveforms with at least one zero and arbitrary $k \geq 2$, whereas Section 6.2 considers cosine waveforms with at least one zero for $k=3$. In Section 7 four case studies of application of descriptions of nonnegative waveforms with fundamental and $k$ th harmonic in PA modelling are presented. In the Appendices, list of some finite sums of trigonometric functions, widely used throughout the paper, and brief account of the Chebyshev polynomials are provided.

\section{Minimum Function, Gain Function, and Conflict Set}

In this section we consider minimum function and gain function (Section 2.1), conflict set (Section 2.2), and parameter space (Section 2.3) in the context of nonnegative waveforms with fundamental and $k$ th harmonic.

We start with provision of a brief account of the facts related to the concepts of minimum function and conflict set. For this purpose let us denote by $f(x ; u)$ a family of smooth functions of $n$ variables depending on $m$ parameters, where $x \in R^{n}$ is $n$-tuple of variables and $u \in R^{m}$ is $m$-tuple of parameters. The minimum function $F: R^{m} \rightarrow R$, associated with the function $f$, is defined as $F(u)=\min _{x} f(x ; u)$. Therefore, the domain of the minimum function is parameter space of the function $f$. The minimum function $F(u)$ is continuous, but not necessarily smooth function of parameters $[13,24]$. It is a smooth function if $f(x ; u)$ possesses unique global minimum at nondegenerate critical point [13] (critical point is degenerate if at least first two consecutive derivatives are equal to zero). In this context, the conflict set can be defined as the set of the parameters for which function $f$ has global minimum at a degenerate critical point or/and multiple global minima [13].

For a wide class of minimum functions, when the number of parameters is not greater than four, the behaviour of minimum function in a neighbourhood of any point can be described by one of "normal forms" from a finite list as stated in [24]. For example, for smooth function $f: R \times R^{2} \rightarrow R$, the minimum function $F\left(u_{1}, u_{2}\right)=\min _{x} f\left(x, u_{1}, u_{2}\right)$ near the origin can be locally reduced to one of the following three normal forms [25]: $-\left|u_{1}\right|, \min \left(u_{1}, u_{2}, u_{1}+u_{2}\right)$, or $\min _{x}\left(x^{4}+\right.$ $\left.u_{1} x^{2}+u_{2} x\right)$. In this example, the conflict set is the set of all points $\left(u_{1}, u_{2}\right)$ for which minimum function $F\left(u_{1}, u_{2}\right)$ is not differentiable because function $f\left(x, u_{1}, u_{2}\right)$ possesses at least two global minima [25]. 
2.1. Minimum Function and Gain Function. In what follows we consider family of waveforms of type

$$
w(\tau ; \gamma, A, \alpha)=1-\gamma(\cos \tau+A \cos (k \tau+\alpha)),
$$

where $\tau$ stands for $\omega t, \gamma>0, k \geq 2, A>0$, and $\alpha \in[0,2 \pi)$. Waveforms of type (1) include all possible shapes which can occur, but not all possible waveforms containing fundamental and $k$ th harmonic. However, shifting of waveforms of type (1) along $\tau$-axis could recover all possible waveforms with fundamental and $k$ th harmonic.

The problem of finding nonnegative waveform of type (1) having maximum amplitude of fundamental harmonic plays an important role in optimization of PA efficiency. This extremal problem can be reformulated as problem of finding nonnegative waveform from family (1) having maximum possible value of coefficient $\gamma$. Nonnegative waveform of family (1) with maximum possible value of coefficient $\gamma$ is called "optimal" or "extremal" waveform.

Furthermore, let us introduce an auxiliary waveform

$$
f(\tau ; A, \alpha)=-\cos \tau-A \cos (k \tau+\alpha),
$$

which is smooth function of one variable $\tau$ and two parameters $A$ and $\alpha$. In terms of $f(\tau ; A, \alpha)$, the above extremal problem reduces to the problem of finding maximum possible value of coefficient $\gamma$ that satisfies

$$
1+\gamma f(\tau ; A, \alpha) \geq 0
$$

Clearly, for any prescribed pair $(A, \alpha)$, there is a unique maximal value of coefficient $\gamma$ for which inequality (3) holds for all $\tau$. This maximal value of $\gamma$ associated with the pair $(A, \alpha)$ we denote it by $G(A, \alpha)$ and call it "gain function."

Let

$$
F_{\min }(A, \alpha)=\min _{\tau} f(\tau ; A, \alpha)
$$

be the minimum function associated with $f(\tau ; A, \alpha)$. According to (3), $G(A, \alpha)$ and $F_{\min }(A, \alpha)$ satisfy the following relation: $1+G(A, \alpha) F_{\min }(A, \alpha)=0$. Since $F_{\min }(A, \alpha)$ is obviously nonzero it follows immediately that

$$
G(A, \alpha)=-\frac{1}{F_{\min }(A, \alpha)} .
$$

A relation analogue to (5), for $k=2$ (fundamental and second harmonic), has been derived in [4]. According to our best knowledge, it was the first appearance of gain function expressed via associated minimum function. The consideration presented in [4] has been restricted to the particular case when $\alpha=\pi$. The same problem for $\alpha=\pi$ and arbitrary $k \geq 2$ has been investigated in [3] (see also [10]).

According to above consideration, the problem of finding 3-tuple $(\gamma, A, \alpha)$ with maximum possible value of $\gamma$ for which (3) holds is equivalent to the problem of finding maximum value of gain function

$$
\gamma_{\max }=\max _{A, \alpha} G(A, \alpha)=-\frac{1}{\max _{A, \alpha} F_{\min }(A, \alpha)}
$$

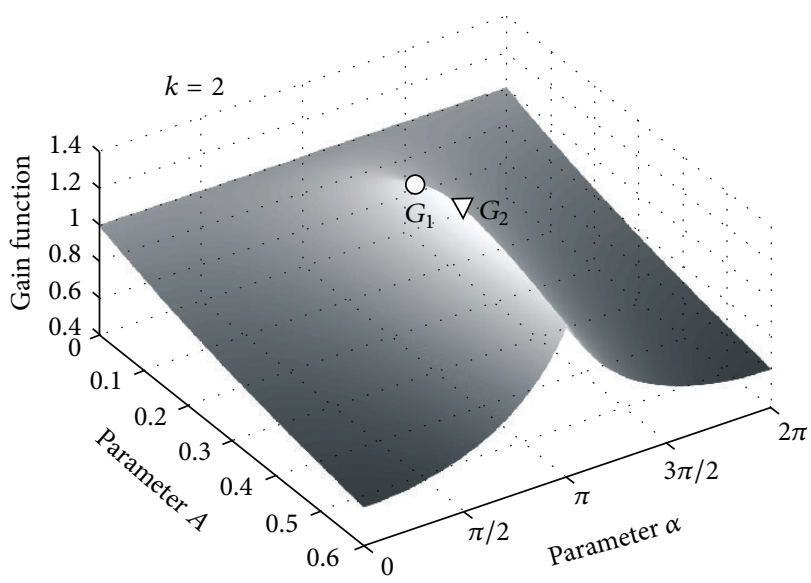

Figure 1: Graph of $G(A, \alpha)$ for $k=2$. Points $G_{1}$ and $G_{2}$ denote beginning of the ridge and maximum of gain function, respectively.

and corresponding pair $\left(A^{*}, \alpha^{*}\right)$ that satisfies

$$
\begin{gathered}
\gamma_{\max }=\max _{A, \alpha} G(A, \alpha)=G\left(A^{*}, \alpha^{*}\right), \\
\max _{A, \alpha} F_{\min }(A, \alpha)=F_{\min }\left(A^{*}, \alpha^{*}\right) .
\end{gathered}
$$

Thus the optimal waveform $w^{*}(\tau)$ is determined by parameters $\gamma_{\max }, A^{*}$, and $\alpha^{*}$; that is,

$$
w^{*}(\tau)=w\left(\tau ; \gamma_{\max }, A^{*}, \alpha^{*}\right)
$$

Optimal waveform has two global minima (this claim will be justified in Section 4, Remark 21). Consequently, the pair $\left(A^{*}, \alpha^{*}\right)$, which corresponds to maximum of gain function $G(A, \alpha)$, belongs to conflict set in $(A, \alpha)$ parameter space.

Figure 1 shows graph of gain function $G(A, \alpha)$ for $k=2$. Notice that it has sharp ridge and that maximum of gain function (point $G_{2}$ ) lies on the ridge. This maximum corresponds to the optimal waveform (solution of the considered extremal problem). The beginning of the ridge (point $G_{1}$ ) corresponds to the waveform which possesses global minimum at degenerate critical point, that is, corresponds to maximally flat waveform (e.g., see [21]). Gain function $G(A, \alpha)$ is not differentiable on the ridge and consequently is not differentiable at the point where it has global maximum. This explains why the approach based on critical points does not work and why conflict set is so important in the considered problem.

Positions of global minima of $f(\tau ; A, \alpha)$ for $k=2$ are presented in Figure 2. According to Proposition 1, conflict set is the ray defined by $A>1 / 4$ and $\alpha=\pi$. Waveforms $f(\tau ; A, \alpha)$ with parameters that belong to the conflict set have two global minima. The waveform corresponding to the end point of the ray $(A=1 / 4$ and $\alpha=\pi)$ has global minimum at degenerate critical point (so-called maximally flat waveform [21]).

Nonnegative waveforms of type (1) with $\gamma=G(A, \alpha)=$ $-1 / F_{\min }(A, \alpha)$ have at least one zero. To show that, it is sufficient to see that $w(\tau ; \gamma, A, \alpha)=0$ for $\tau$ satisfying $f(\tau$; $A, \alpha)=F_{\text {min }}(A, \alpha)$. 


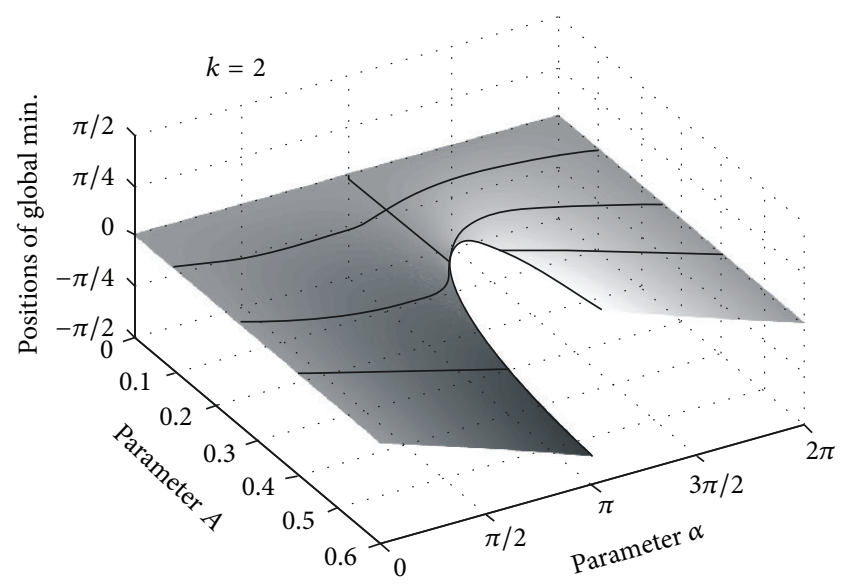

Figure 2: Positions of global minima of $f(\tau ; A, \alpha)$ for $k=2$.

The problem of finding maximum value of fundamental harmonic cosine part of nonnegative waveform of the form

$$
w_{a}\left(\tau ; \gamma_{a}, b, A, \alpha\right)=1-\gamma_{a}(\cos \tau+b \sin \tau+A \cos (k \tau+\alpha)) \text {, }
$$

where $\gamma_{a}>0$, is also related to the problem of finding maximum of the minimum function. Optimal waveform of family (9) has two global minima (this claim will be justified in Section 5, Remark 25), and therefore corresponding 3-tuple of parameters belongs to the conflict set in parameter space of family (9).

Let us introduce an auxiliary waveform

$$
f_{a}(\tau ; b, A, \alpha)=-\cos \tau-b \sin \tau-A \cos (k \tau+\alpha),
$$

and corresponding minimum function $F_{a, \min }(b, A, \alpha)=$ $\min _{\tau} f_{a}(\tau ; b, A, \alpha)$. Inequality $w_{a}\left(\tau ; \gamma_{a}, b, A, \alpha\right) \geq 0$ can be rewritten as $1+\gamma_{a} F_{a, \min }(b, A, \alpha) \geq 0$ and therefore the highest value of $\gamma_{a}$ is attained for $\gamma_{a}=-1 / F_{a, \min }(b, A, \alpha)$. It immediately follows that nonnegative waveform of type (9) with $\gamma_{a}=-1 / F_{a, \min }(b, A, \alpha)$ has zero for $\tau$ satisfying $f_{a}(\tau$; $b, A, \alpha)=F_{a, \min }(b, A, \alpha)$.

2.2. Conflict Set. Historically, conflict set came into being from the problems in which families of smooth functions (such as potentials, distances, and waveforms) with two competing minima occur. The situation when competing minima become equal refers to the presence of conflict set (Maxwell set, Maxwell strata) in the associated parameter space.

There are many facets of conflict set. For example, in the problem involving distances between two sets of points, the conflict set is the intersections between iso-distance lines [14]. Conflict set also arises in the situation when two wave fronts coming from different objects meet $[15,25]$. In the study of black holes, conflict set is the line of crossover of the horizon formed by the merger of two black holes [19]. In the classical Euler problem, conflict set is a set of points where distinct extremal trajectories with the same value of the cost functional meet one another [18].
Conflict set is very difficult to calculate, both analytically and numerically (e.g., see [15]), because of apparent nondifferentiability in some directions. In optimization of PA efficiency, some authors already reported difficulties in finding optimum via standard analytical tools $[4,5]$.

In this section, we consider conflict set in the context of family of waveforms of type (2) for arbitrary $k \geq 2$. In this context, for prescribed integer $k \geq 2$, conflict set is said to be a set of all pairs $(A, \alpha)$ for which $f(\tau ; A, \alpha)$ possesses multiple global minima.

Suppose that $\tau^{\prime}$ and $\tau^{\prime \prime}$ are the positions of global minima of $f(\tau ; A, \alpha)$. Then, the conflict set is specified by the following set of relations:

$$
\begin{gathered}
f\left(\tau^{\prime} ; A, \alpha\right)=f\left(\tau^{\prime \prime} ; A, \alpha\right), \\
f^{\prime}\left(\tau^{\prime} ; A, \alpha\right)=0, \quad f^{\prime}\left(\tau^{\prime \prime} ; A, \alpha\right)=0, \\
f^{\prime \prime}\left(\tau^{\prime} ; A, \alpha\right)>0, \quad f^{\prime \prime}\left(\tau^{\prime \prime} ; A, \alpha\right)>0, \\
(\forall \tau) f(\tau ; A, \alpha) \geq f\left(\tau^{\prime} ; A, \alpha\right) .
\end{gathered}
$$

Relations (12) and (13) say that $f(\tau ; A, \alpha)$ has minima at $\tau^{\prime}$ and $\tau^{\prime \prime}$, while relations (11) and (14) imply that these minima are equal and global.

The following proposition describes the conflict set of family of waveforms of type (2).

Proposition 1. Conflict set of family of waveforms of type (2) is the set of all pairs $(A, \alpha)$ such that $A>1 / k^{2}$ and $\alpha=\pi$.

The proof of Proposition 1, which is provided at the end of this section, also implies that the following four corollaries hold.

Corollary 2. The conflict set has end point at $(A, \alpha)=$ $\left(1 / k^{2}, \pi\right)$. This end point corresponds to the maximally flat waveform [21].

Corollary 3. Waveforms of type (2) with parameters that belong to conflict set have two global minima at $\pm \tau_{\Delta}$, where $0<\tau_{\Delta} \leq \pi / k$.

Corollary 4. Every waveform with fundamental and $k$ th harmonic has either one or two global minima.

Corollary 5. Conflict set can be parameterised in terms of $\tau_{\Delta}$ as follows:

$$
\alpha=\pi, \quad A\left(\tau_{\Delta}\right)=\frac{\sin \tau_{\Delta}}{k \sin k \tau_{\Delta}}, \quad 0<\tau_{\Delta} \leq \frac{\pi}{k} .
$$

Notice that $A\left(\tau_{\Delta}\right)$ is monotonically increasing function on interval $0<\tau_{\Delta} \leq \pi / k$.

Proof of Proposition 1. Without loss of generality, we can restrict our consideration to the interval $-\pi<\tau \leq \pi$. This is an immediate consequence of the fact that $f(\tau ; A, \alpha)$ is a periodic function.

Suppose that $\tau^{\prime}$ and $\tau^{\prime \prime}$, where $\tau^{\prime}<\tau^{\prime \prime}$, are points at which $f(\tau ; A, \alpha)$ has two equal global minima. Then conflict set is 
specified by relations (11)-(14). From (11)-(13) it follows that relations

$$
\begin{gathered}
f\left(\tau^{\prime} ; A, \alpha\right)-f\left(\tau^{\prime \prime} ; A, \alpha\right)=0, \\
f^{\prime}\left(\tau^{\prime} ; A, \alpha\right)+f^{\prime}\left(\tau^{\prime \prime} ; A, \alpha\right)=0, \\
f^{\prime}\left(\tau^{\prime} ; A, \alpha\right)-f^{\prime}\left(\tau^{\prime \prime} ; A, \alpha\right)=0, \\
f^{\prime \prime}\left(\tau^{\prime} ; A, \alpha\right)+f^{\prime \prime}\left(\tau^{\prime \prime} ; A, \alpha\right)>0
\end{gathered}
$$

also hold. Let

$$
\tau_{s r}=\frac{\left(\tau^{\prime}+\tau^{\prime \prime}\right)}{2}, \quad \tau_{\Delta}=\frac{\left(\tau^{\prime \prime}-\tau^{\prime}\right)}{2}
$$

be a pair of points associated with $\left(\tau^{\prime}, \tau^{\prime \prime}\right)$. Clearly

$$
\begin{gathered}
-\pi<\tau_{s r}<\pi, \\
0<\tau_{\Delta}<\pi, \\
\tau^{\prime \prime}=\tau_{s r}+\tau_{\Delta}, \quad \tau^{\prime}=\tau_{s r}-\tau_{\Delta} .
\end{gathered}
$$

The first and second derivatives of $f(\tau ; A, \alpha)$ are equal to

$$
\begin{gathered}
f^{\prime}(\tau ; A, \alpha)=\sin \tau+k A \sin (k \tau+\alpha), \\
f^{\prime \prime}(\tau ; A, \alpha)=\cos \tau+k^{2} A \cos (k \tau+\alpha) .
\end{gathered}
$$

By using (20)-(21), system (16) can be rewritten as

$$
\begin{array}{r}
\sin \tau_{s r} \sin \tau_{\Delta}+A \sin \left(k \tau_{s r}+\alpha\right) \sin k \tau_{\Delta}=0, \\
\sin \tau_{s r} \cos \tau_{\Delta}+k A \sin \left(k \tau_{s r}+\alpha\right) \cos k \tau_{\Delta}=0, \\
\cos \tau_{s r} \sin \tau_{\Delta}+k A \cos \left(k \tau_{s r}+\alpha\right) \sin k \tau_{\Delta}=0, \\
\cos \tau_{s r} \cos \tau_{\Delta}+k^{2} A \cos \left(k \tau_{s r}+\alpha\right) \cos k \tau_{\Delta}>0 .
\end{array}
$$

From (19) it follows that $\sin \tau_{\Delta}>0$. Multiplying (24) and (25) with $-\cos \tau_{\Delta}$ and $\sin \tau_{\Delta}>0$, respectively, and summing the resulting relations, we obtain $k A \cos \left(k \tau_{s r}+\right.$ $\alpha)\left[k \sin \tau_{\Delta} \cos k \tau_{\Delta}-\sin k \tau_{\Delta} \cos \tau_{\Delta}\right]>0$. The latest relation immediately implies that

$$
k \sin \tau_{\Delta} \cos k \tau_{\Delta}-\sin k \tau_{\Delta} \cos \tau_{\Delta} \neq 0 .
$$

Equations (22) and (23) can be considered as a system of two linear equations in terms of $\sin \tau_{s r}$ and $A \sin \left(k \tau_{s r}+\alpha\right)$. According to (26), the determinant of this system is nonzero and therefore it has only trivial solution:

$$
\sin \tau_{s r}=0, \quad \sin \left(k \tau_{s r}+\alpha\right)=0 .
$$

According to (18), $\sin \tau_{s r}=0$ implies

$$
\tau_{s r}=0 .
$$

According to (20), $\tau_{s r}=0$ implies

$$
\tau^{\prime \prime}=\tau_{\Delta}, \quad \tau^{\prime}=-\tau_{\Delta} .
$$

Furthermore, $\tau_{s r}=0$ and $\sin \left(k \tau_{s r}+\alpha\right)=0$ imply that $\sin \alpha=$ 0 . From (29) it follows that $\tau_{\Delta}$ is position of global minimum of $f(\tau ; A, \alpha)$. Clearly $f\left(\tau_{\Delta} ; A, \alpha\right) \leq f(0 ; A, \alpha)$, which together with $\sin \alpha=0$ leads to

$$
1-\cos \tau_{\Delta}+A \cos \alpha\left(1-\cos k \tau_{\Delta}\right) \leq 0
$$

From $\tau_{\Delta} \neq 0$ (see (19)), $A>0$ and (30) it follows that $\cos \alpha<$ 0 , which together with $\sin \alpha=0$ yields

$$
\alpha=\pi
$$

Since $\tau_{\Delta}$ is position of global minimum, it follows that $f\left(\tau_{\Delta} ; A, \pi\right) \leq f(\pi / k ; A, \pi)$. Accordingly $A\left(1+\cos k \tau_{\Delta}\right) \leq$ $\cos \tau_{\Delta}-\cos \pi / k$, which together with $A>0$ implies that $\cos \tau_{\Delta}-\cos \pi / k \geq 0$. This relation along with (19) yields

$$
0<\tau_{\Delta} \leq \frac{\pi}{k}
$$

Substitution of (31) and (28) in (24) leads to

$$
A=\frac{\sin \tau_{\Delta}}{k \sin k \tau_{\Delta}} .
$$

Notice that $\sin k \tau_{\Delta} / \sin \tau_{\Delta}$ is monotonically decreasing function on interval (32). Therefore parameter $A$ is monotonically increasing function on the same interval with $\lim _{\tau_{\Delta} \rightarrow 0+} A=$ $1 / k^{2}$. Consequently $A>1 / k^{2}$, which completes the proof.

2.3. Parameter Space. In parameter space of family of waveforms (2) there are two subsets playing important role in the classification of the family instances. These are conflict set and catastrophe set.

Catastrophe set is subset of parameter space of waveform $f(\tau ; A, \alpha)$. It consists of those pairs $(A, \alpha)$ for which the corresponding waveforms $f(\tau ; A, \alpha)$ have degenerate critical points at which first and second derivatives are equal to zero. Thus, for finding catastrophe set we have to consider the following system of equations:

$$
\begin{aligned}
& f^{\prime}\left(\tau_{d} ; A, \alpha\right)=0, \\
& f^{\prime \prime}\left(\tau_{d} ; A, \alpha\right)=0,
\end{aligned}
$$

where $\tau_{d}$ is a degenerate critical point of waveform $f(\tau ; A, \alpha)$.

Conflict set in parameter space of waveform $f(\tau ; A, \alpha)$, as shown in Proposition 1, is the ray described by $A>1 / k^{2}$ and $\alpha=\pi$. It is intimately connected to catastrophe set.

In what follows in this subsection we use polar coordinate system $(A \cos \alpha, A \sin \alpha)$ instead of Cartesian coordinate system $(A, \alpha)$. Examples of catastrophe set and conflict set for $k \leq 5$ plotted in parameter space $(A \cos \alpha, A \sin \alpha)$ are presented in Figure 3. Solid line represents the catastrophe set while dotted line describes conflict set. The isolated pick points (usually called cusp) which appear in catastrophe curves correspond to maximally flat waveforms, with maximally flat minimum and/or maximally flat maximum. There are two such picks in the catastrophe curves for $k=2$ and 

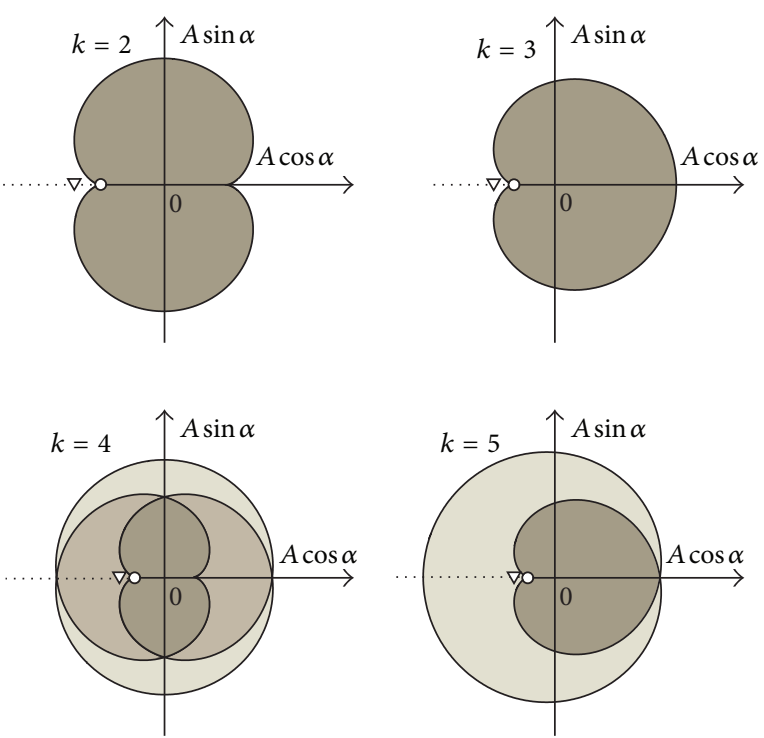

FIGURE 3: Catastrophe set (solid line) and corresponding conflict set (dotted line) for $k \leq 5$. In each plot, white triangle dot corresponds to optimal waveform and white circle dot corresponds to maximally flat waveform.

$k=4$ and one in the catastrophe curves for $k=3$ and $k=5$. Notice that the end point of conflict set is the cusp point.

Catastrophe set divides the parameter space $(A \cos \alpha$, $A \sin \alpha)$ into disjoint subsets. In the cases $k=2$ and $k=$ 3 catastrophe curve defines inner and outer part. For $k>$ 3 catastrophe curve makes partition of parameter space in several inner subsets and one outer subset (see Figure 3 ).

Notice also that multiplying $f(\tau ; A, \alpha)$ with a positive constant and adding in turn another constant, which leads to waveform of type $w(\tau ; \gamma, A, \alpha)$ (see (1) and (2)), do not make impact on the character of catastrophe and conflict sets. This is because in the course of finding catastrophe set first and second derivatives of $f(\tau ; A, \alpha)$ are set to zero. Clearly (34) in terms of $f(\tau ; A, \alpha)$ are equivalent to the analogous equations in terms of $w(\tau ; \gamma, A, \alpha)$. Analogously, in the course of finding conflict set we consider only the positions of global minima (these positions for waveforms $f(\tau ; A, \alpha)$ and $w(\tau ; \gamma, A, \alpha)$ are the same).

\section{Nonnegative Waveforms with at Least One Zero}

In what follows let us consider a waveform containing $\mathrm{dc}$ component, fundamental and $k$ th $(k \geq 2)$ harmonic of the form

$$
T_{k}(\tau)=1+a_{1} \cos \tau+b_{1} \sin \tau+a_{k} \cos k \tau+b_{k} \sin k \tau .
$$

The amplitudes of fundamental and $k$ th harmonic of waveform of type (35), respectively, are

$$
\begin{aligned}
& \lambda_{1}=\sqrt{a_{1}^{2}+b_{1}^{2}}, \\
& \lambda_{k}=\sqrt{a_{k}^{2}+b_{k}^{2}} .
\end{aligned}
$$

As it is shown in Section 2.1, nonnegative waveforms with maximal amplitude of fundamental harmonic or maximal coefficient of fundamental harmonic cosine part have at least one zero. It is also shown in Section 2.2 (Corollary 4) that waveforms of type (35) with nonzero amplitude of fundamental harmonic have either one or two global minima. Consequently, if nonnegative waveform of type (35) with nonzero amplitude of fundamental harmonic has at least one zero, then it has at most two zeros.

In Section 3.1 we provide general description of nonnegative waveforms of type (35) with at least one zero. In Sections 3.2 and 3.3 we consider nonnegative waveforms of type (35) with two zeros.

3.1. General Description of Nonnegative Waveforms with at Least One Zero. The main result of this section is presented in the following proposition.

Proposition 6. Every nonnegative waveform of type (35) with at least one zero can be expressed in the following form:

$$
T_{k}(\tau)=\left[1-\cos \left(\tau-\tau_{0}\right)\right]\left[1-\lambda_{k} r_{k}(\tau)\right],
$$

where

$$
\begin{aligned}
r_{k}(\tau)= & (k-1) \cos \xi \\
& +2 \sum_{n=1}^{k-1}(k-n) \cos \left(n\left(\tau-\tau_{0}\right)+\xi\right),
\end{aligned}
$$

providing that

$$
\begin{gathered}
\lambda_{k} \leq\left[(k-1) \cos \xi+k \frac{\sin (\xi-\xi / k)}{\sin (\xi / k)}\right]^{-1}, \\
|\xi| \leq \pi .
\end{gathered}
$$

Remark 7. Function on the right hand side of (40) is monotonically increasing function of $|\xi|$ on interval $|\xi| \leq \pi$ (for more details about this function see Remark 15). From (57) and (65) it follows that relation

$$
0 \leq \lambda_{k} \leq 1
$$

holds for every nonnegative waveform of type (35). Notice that, according to (40), $\lambda_{k}=1$ implies $|\xi|=\pi$. Substitution of $\lambda_{k}=1$ and $|\xi|=\pi$ into (55) yields $T_{k}(\tau)=1-$ $\cos k\left(\tau-\tau_{0}\right)$. Consequently, $\lambda_{k}=1$ implies that amplitude $\lambda_{1}$ of fundamental harmonic is equal to zero.

Remark 8. Conversion of (38) into additive form leads to the following expressions for coefficients of nonnegative waveforms of type (35) with at least one zero:

$$
\begin{gathered}
a_{1}=-\left(1+\lambda_{k} \cos \xi\right) \cos \tau_{0}-k \lambda_{k} \sin \xi \sin \tau_{0}, \\
b_{1}=-\left(1+\lambda_{k} \cos \xi\right) \sin \tau_{0}+k \lambda_{k} \sin \xi \cos \tau_{0}, \\
a_{k}=\lambda_{k} \cos \left(k \tau_{0}-\xi\right), \\
b_{k}=\lambda_{k} \sin \left(k \tau_{0}-\xi\right),
\end{gathered}
$$

providing that $\lambda_{k}$ satisfy (40) and $|\xi| \leq \pi$. 


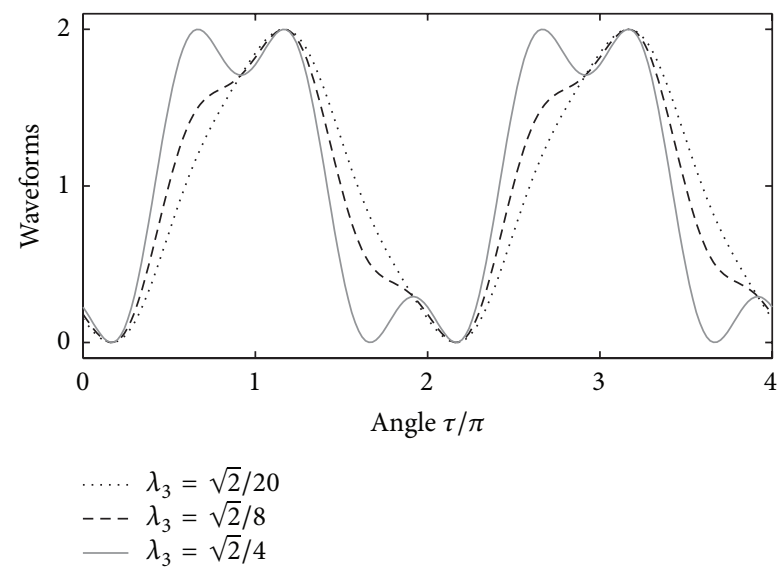

FIgURE 4: Nonnegative waveforms with at least one zero for $k=3$, $\tau_{0}=\pi / 6$, and $\xi=3 \pi / 4$.

Three examples of nonnegative waveforms with at least one zero for $k=3$ are presented in Figure 4 (examples of nonnegative waveforms with at least one zero for $k=2$ can be found in [12]). For all three waveforms presented in Figure 4, we assume that $\tau_{0}=\pi / 6$ and $\xi=3 \pi / 4$. From (40) it follows that $\lambda_{3} \leq \sqrt{2} / 4$. Coefficients of waveform with $\lambda_{3}=\sqrt{2} / 20$ (dotted line) are $a_{1}=-0.8977, b_{1}=-0.3451, a_{3}=0.05$, and $b_{3}=-0.05$. Coefficients of waveform with $\lambda_{3}=\sqrt{2} / 8$ (dashed line) are $a_{1}=-0.9453, b_{1}=-0.1127, a_{3}=0.125$, and $b_{3}=-0.125$. Coefficients of waveform with $\lambda_{3}=\sqrt{2} / 4$ (solid line) are $a_{1}=-1.0245, b_{1}=0.2745, a_{3}=0.25$, and $b_{3}=-0.25$. First two waveforms have one zero, while third waveform (presented with solid line) has two zeros.

Proof of Proposition 6. Waveform of type (35), containing dc component, fundamental and $k$ th harmonic, can be also expressed in the form

$$
T_{k}(\tau)=1+\lambda_{1} \cos \left(\tau+\varphi_{1}\right)+\lambda_{k} \cos \left(k \tau+\varphi_{k}\right),
$$

where $\lambda_{1} \geq 0, \lambda_{k} \geq 0, \varphi_{1} \in(-\pi, \pi]$, and $\varphi_{k} \in(-\pi, \pi]$. It is easy to see that relations between coefficient of (35) and parameters of (47) read as follows:

$$
\begin{array}{ll}
a_{1}=\lambda_{1} \cos \varphi_{1}, & b_{1}=-\lambda_{1} \sin \varphi_{1}, \\
a_{k}=\lambda_{k} \cos \varphi_{k}, & b_{k}=-\lambda_{k} \sin \varphi_{k} .
\end{array}
$$

Let us introduce $\xi$ such that

$$
|\xi| \leq \pi, \quad \xi=\left(k \tau_{0}+\varphi_{k}\right) \bmod 2 \pi .
$$

Using (50), coefficients (49) can be expressed as (45)-(46).

Let us assume that $T_{k}(\tau)$ is nonnegative waveform of type (35) with at least one zero; that is, $T_{k}(\tau) \geq 0$ and $T_{k}\left(\tau_{0}\right)=0$ for some $\tau_{0}$. Notice that conditions $T_{k}(\tau) \geq 0$ and $T_{k}\left(\tau_{0}\right)=0$ imply that $T_{k}^{\prime}\left(\tau_{0}\right)=0$. From $T_{k}\left(\tau_{0}\right)=0$ and $T_{k}^{\prime}\left(\tau_{0}\right)=0$, by using (50), it follows that

$$
\begin{gathered}
\lambda_{1} \cos \left(\tau_{0}+\varphi_{1}\right)=-\left(1+\lambda_{k} \cos \xi\right), \\
\lambda_{1} \sin \left(\tau_{0}+\varphi_{1}\right)=-k \lambda_{k} \sin \xi
\end{gathered}
$$

respectively. On the other hand, $\lambda_{1} \cos \left(\tau+\varphi_{1}\right)$ can be rewritten as

$$
\begin{aligned}
\lambda_{1} \cos \left(\tau+\varphi_{1}\right)= & \lambda_{1} \cos \left(\tau_{0}+\varphi_{1}\right) \cos \left(\tau-\tau_{0}\right) \\
& -\lambda_{1} \sin \left(\tau_{0}+\varphi_{1}\right) \sin \left(\tau-\tau_{0}\right) .
\end{aligned}
$$

Substitution of (51) into (52) yields

$$
\begin{aligned}
\lambda_{1} \cos \left(\tau+\varphi_{1}\right)= & -\left(1+\lambda_{k} \cos \xi\right) \cos \left(\tau-\tau_{0}\right) \\
& +k \lambda_{k} \sin \xi \sin \left(\tau-\tau_{0}\right) .
\end{aligned}
$$

According to (50), it follows that $\cos \left(k \tau+\varphi_{k}\right)=\cos (k(\tau-$ $\left.\left.\tau_{0}\right)+\xi\right)$; that is,

$$
\cos \left(k \tau+\varphi_{k}\right)=\cos \xi \cos k\left(\tau-\tau_{0}\right)-\sin \xi \sin k\left(\tau-\tau_{0}\right) .
$$

Furthermore, substitution of (54) and (53) into (47) leads to

$$
\begin{aligned}
T_{k}(\tau)= & {\left[1-\cos \left(\tau-\tau_{0}\right)\right]\left[1+\lambda_{k} \cos \xi\right] } \\
& -\lambda_{k}\left[1-\cos k\left(\tau-\tau_{0}\right)\right] \cos \xi \\
& +\lambda_{k}\left[k \sin \left(\tau-\tau_{0}\right)-\sin k\left(\tau-\tau_{0}\right)\right] \sin \xi .
\end{aligned}
$$

According to (A.2) and (A.4) (see Appendices), there is common factor $\left[1-\cos \left(\tau-\tau_{0}\right)\right]$ for all terms in (55). Consequently, (55) can be written in the form (38), where

$$
\begin{aligned}
r_{k}(\tau)= & -\cos \xi+\left[\frac{1-\cos k\left(\tau-\tau_{0}\right)}{1-\cos \left(\tau-\tau_{0}\right)}\right] \cos \xi \\
& -\left[\frac{k \sin \left(\tau-\tau_{0}\right)-\sin k\left(\tau-\tau_{0}\right)}{1-\cos \left(\tau-\tau_{0}\right)}\right] \sin \xi .
\end{aligned}
$$

From (56), by using (A.2), (A.4), and $\cos \xi \cos n\left(\tau-\tau_{0}\right)-$ $\sin \xi \sin n\left(\tau-\tau_{0}\right)=\cos \left(n\left(\tau-\tau_{0}\right)+\xi\right)$, we obtain (39).

In what follows we are going to prove that (40) also holds. According to (38), $T_{k}(\tau)$ is nonnegative if and only if

$$
\lambda_{k} \max _{\tau} r_{k}(\tau) \leq 1
$$

Let us first show that position of global maximum of $r_{k}(\tau)$ belongs to the interval $\left|\tau-\tau_{0}\right| \leq 2 \pi / k$. Relation (56) can be rewritten as

$$
r_{k}(\tau)=r_{k}\left(\tau_{0}-\frac{2 \xi}{k}\right)+q_{k}(\tau)
$$

where

$$
\begin{aligned}
r_{k}\left(\tau_{0}-\frac{2 \xi}{k}\right)=(k-1) \cos \xi+k \frac{\sin (\xi-\xi / k)}{\sin (\xi / k)} \\
q_{k}(\tau)=\frac{1}{1-\cos \left(\tau-\tau_{0}\right)} \\
\cdot\left[\cos \xi-\cos \left(k\left(\tau-\tau_{0}+\frac{\xi}{k}\right)\right)\right. \\
\left.\quad+\frac{k \sin \xi}{\sin (\xi / k)}\left(\cos \left(\tau-\tau_{0}+\frac{\xi}{k}\right)-\cos \left(\frac{\xi}{k}\right)\right)\right] .
\end{aligned}
$$


For $|\xi|<\pi$, relation $\sin \xi / \sin (\xi / k)>0$ obviously holds. From $\cos t>\cos t^{\prime}$ for $|t| \leq \pi / k<\left|t^{\prime}\right| \leq \pi$ it follows that position of global maximum of the function of type $[c \cos t-\cos (k t)]$ for $c>0$ belongs to interval $|t| \leq \pi / k$. Therefore position of global maximum of the expression in the square brackets in (60) for $|\xi|<\pi$ belongs to interval $\left|\tau-\tau_{0}+\xi / k\right| \leq \pi / k$. This inequality together with $|\xi|<\pi$ leads to $\left|\tau-\tau_{0}\right|<2 \pi / k$. Since $\left[1-\cos \left(\tau-\tau_{0}\right)\right]^{-1}$ decreases with increasing $\left|\tau-\tau_{0}\right| \leq \pi$, it follows that $q_{k}(\tau)$ for $|\xi|<\pi$ has global maximum on interval $\left|\tau-\tau_{0}\right|<2 \pi / k$. For $|\xi|=\pi$, it is easy to show that $\max _{\tau} q_{k}(\tau)=$ $q_{k}\left(\tau_{0} \pm 2 \pi / k\right)=0$. Since $r_{k}(\tau)-q_{k}(\tau)$ is constant (see (58)), it follows from previous considerations that $r_{k}(\tau)$ has global maximum on interval $\left|\tau-\tau_{0}\right| \leq 2 \pi / k$.

To find $\max _{\tau} r_{k}(\tau)$, let us consider first derivative of $r_{k}(\tau)$ with respect to $\tau$. Starting from (56), first derivative of $r_{k}(\tau)$ can be expressed in the following form:

$$
\frac{d r_{k}(\tau)}{d \tau}=-s(\tau) \cdot \sin \left(\frac{k\left(\tau-\tau_{0}\right)}{2}+\xi\right),
$$

where

$$
\begin{aligned}
s(\tau)= & {\left[\sin \left(\frac{k\left(\tau-\tau_{0}\right)}{2}\right) \cos \left(\frac{\tau-\tau_{0}}{2}\right)\right.} \\
& \left.-k \cos \left(\frac{k\left(\tau-\tau_{0}\right)}{2}\right) \sin \left(\frac{\tau-\tau_{0}}{2}\right)\right] \\
& \cdot \sin ^{-3}\left(\frac{\tau-\tau_{0}}{2}\right) .
\end{aligned}
$$

Using (A.6) (see Appendices), (62) can be rewritten as

$$
s(\tau)=2 \sum_{n=1}^{k-1} n(k-n) \cos \left(\frac{(k-2 n)\left(\tau-\tau_{0}\right)}{2}\right) .
$$

From $n(k-n)>0$ and $|k-2 n|<k, n=1, \ldots,(k-1)$, it follows that all summands in (63) decrease with increasing $\left|\tau-\tau_{0}\right|$ providing that $\left|\tau-\tau_{0}\right| \leq 2 \pi / k$. Therefore $s(\tau) \geq s\left(\tau_{0} \pm\right.$ $2 \pi / k)=k / \sin ^{2}(\pi / k)>0$ for $\left|\tau-\tau_{0}\right| \leq 2 \pi / k$. Consequently, $d r_{k}(\tau) / d \tau=0$ and $\left|\tau-\tau_{0}\right| \leq 2 \pi / k$ imply that $\sin \left(k\left(\tau-\tau_{0}\right) / 2+\right.$ $\xi)=0$.

From $|\xi| \leq \pi,\left|\tau-\tau_{0}\right| \leq 2 \pi / k$, and $\sin \left(k\left(\tau-\tau_{0}\right) / 2+\xi\right)=0$ it follows that $\tau-\tau_{0}+\xi / k=-\xi / k$ or $\left|\tau-\tau_{0}+\xi / k\right|=(2 \pi-|\xi|) / k$, and therefore $\cos \left(k\left(\tau-\tau_{0}+\xi / k\right)\right)=\cos \xi$. Since $\cos (\xi / k) \geq$ $\cos (2 \pi-|\xi|) / k$, it follows that $\max _{\tau} q_{k}(\tau)$ is attained for $\tau=$ $\tau_{0}-2 \xi / k$. Furthermore, from (60) it follows that $\max _{\tau} q_{k}(\tau)=$ $q_{k}\left(\tau_{0}-2 \xi / k\right)=0$, which together with (58)-(59) leads to

$$
\begin{aligned}
\max _{\tau} r_{k}(\tau) & =r_{k}\left(\tau_{0}-\frac{2 \xi}{k}\right) \\
& =(k-1) \cos \xi+k \frac{\sin (\xi-\xi / k)}{\sin (\xi / k)} .
\end{aligned}
$$

Both terms on the right hand side of (64) are even functions of $\xi$ and decrease with increase of $|\xi|,|\xi| \leq \pi$. Therefore, $\max _{\tau} r_{k}(\tau)$ attains its lowest value for $|\xi|=\pi$. It is easy to show that right hand side of (64) for $|\xi|=\pi$ is equal to 1 , which further implies that

$$
\max _{\tau} r_{k}(\tau) \geq 1
$$

From (65), it follows that (57) can be rewritten as $\lambda_{k} \leq$ $\left[\max _{\tau} r_{k}(\tau)\right]^{-1}$. Finally, substitution of (64) into $\lambda_{k} \leq$ $\left[\max _{\tau} r_{k}(\tau)\right]^{-1}$ leads to (40), which completes the proof.

3.2. Nonnegative Waveforms with Two Zeros. Nonnegative waveforms of type (35) with two zeros always possess two global minima. Such nonnegative waveforms are therefore related to the conflict set.

In this subsection we provide general description of nonnegative waveforms of type (35) for $k \geq 2$ and exactly two zeros. According to Remark 7, $\lambda_{k}=1$ implies $|\xi|=\pi$ and $T_{k}(\tau)=1-\cos k\left(\tau-\tau_{0}\right)$. Number of zeros of $T_{k}(\tau)=1-$ $\cos k\left(\tau-\tau_{0}\right)$ on fundamental period equals $k$, which is greater than two for $k>2$ and equal to two for $k=2$. In the following proposition we exclude all waveforms with $\lambda_{k}=1$ (the case when $k=2$ and $\lambda_{2}=1$ is going to be discussed in Remark 10).

Proposition 9. Every nonnegative waveform of type (35) with exactly two zeros can be expressed in the following form:

$$
\begin{aligned}
T_{k}(\tau)= & \lambda_{k}\left[1-\cos \left(\tau-\tau_{0}\right)\right]\left[1-\cos \left(\tau-\tau_{0}+\frac{2 \xi}{k}\right)\right] \\
\cdot & {\left[c_{0}+2 \sum_{n=1}^{k-2} c_{n} \cos n\left(\tau-\tau_{0}+\frac{\xi}{k}\right)\right], }
\end{aligned}
$$

where

$$
\begin{aligned}
& c_{n}= {\left[\sin \left(\xi-\frac{n \xi}{k}\right) \cos \left(\frac{\xi}{k}\right)\right.} \\
&\left.-(k-n) \cos \left(\xi-\frac{n \xi}{k}\right) \sin \left(\frac{\xi}{k}\right)\right] \\
& \cdot \sin ^{-3}\left(\frac{\xi}{k}\right), \\
& \lambda_{k}= {\left[(k-1) \cos \xi+k \frac{\sin (\xi-\xi / k)}{\sin (\xi / k)}\right]^{-1}, } \\
& 0<|\xi|<\pi .
\end{aligned}
$$

Remark 10. For $k=2$ waveforms with $\lambda_{2}=1$ also have exactly two zeros. These waveforms can be included in above proposition by substituting (69) with $0<|\xi| \leq \pi$.

Remark 11. Apart from nonnegative waveforms of type (35) with two zeros, there are another two types of nonnegative waveforms which can be obtained from (66)-(68). These are

(i) nonnegative waveforms with $k$ zeros (corresponding to $|\xi|=\pi$ ) and

(ii) maximally flat nonnegative waveforms (corresponding to $\xi=0)$.

Notice that nonnegative waveforms of type (35) with $\lambda_{k}=1$ can be obtained from (66)-(68) by setting $|\xi|=$ $\pi$. Substitution of $\lambda_{k}=1$ and $|\xi|=\pi$ into (66), along with execution of all multiplications and usage of (A.2) (see Appendices), leads to $T_{k}(\tau)=1-\cos k\left(\tau-\tau_{0}\right)$. 
Also, maximally flat nonnegative waveforms (they have only one zero [21]) can be obtained from (66)-(68) by setting $\xi=0$. Thus, substitution of $\xi=0$ into (66)-(68) leads to the following form of maximally flat nonnegative waveform of type (35):

$$
\begin{aligned}
T_{k}(\tau)= & \frac{\left[1-\cos \left(\tau-\tau_{0}\right)\right]^{2}}{3\left(k^{2}-1\right)} \\
& \cdot\left[k\left(k^{2}-1\right)\right. \\
& \left.+2 \sum_{n=1}^{k-2}(k-n)\left((k-n)^{2}-1\right) \cos n\left(\tau-\tau_{0}\right)\right] .
\end{aligned}
$$

Maximally flat nonnegative waveforms of type (35) for $k \leq 4$ can be expressed as

$$
\begin{aligned}
T_{2}(\tau)= & \frac{2}{3}\left[1-\cos \left(\tau-\tau_{0}\right)\right]^{2}, \\
T_{3}(\tau)= & \frac{1}{2}\left[1-\cos \left(\tau-\tau_{0}\right)\right]^{2}\left[2+\cos \left(\tau-\tau_{0}\right)\right], \\
T_{4}(\tau)= & \frac{4}{15}\left[1-\cos \left(\tau-\tau_{0}\right)\right]^{2} \\
& \cdot\left[5+4 \cos \left(\tau-\tau_{0}\right)+\cos 2\left(\tau-\tau_{0}\right)\right] .
\end{aligned}
$$

Remark 12. Every nonnegative waveform of type (35) with exactly one zero at nondegenerate critical point can be described as in Proposition 6 providing that symbol " $\leq$ " in relation (40) is replaced with " $<$ ". This is an immediate consequence of Propositions 6 and 9 and Remark 11.

Remark 13. Identity $\left[1-\cos \left(\tau-\tau_{0}\right)\right]\left[1-\cos \left(\tau-\tau_{0}+2 \xi / k\right)\right]=$ $\left[\cos \xi / k-\cos \left(\tau-\tau_{0}+\xi / k\right)\right]^{2}$ implies that (66) can be also rewritten as

$$
\begin{aligned}
T_{k}(\tau)= & \lambda_{k}\left[\frac{\cos \xi}{k}-\cos \left(\tau-\tau_{0}+\frac{\xi}{k}\right)\right]^{2} \\
& \cdot\left[c_{0}+2 \sum_{n=1}^{k-2} c_{n} \cos n\left(\tau-\tau_{0}+\frac{\xi}{k}\right)\right] .
\end{aligned}
$$

Furthermore, substitution of (67) into (72) leads to

$$
\begin{aligned}
& T_{k}(\tau) \\
& =\lambda_{k}\left[\frac{\cos \xi}{k}-\cos \left(\tau-\tau_{0}+\frac{\xi}{k}\right)\right] \\
& \quad\left[\frac{(k-1) \sin \xi}{\sin (\xi / k)}-2 \sum_{n=1}^{k-1} \frac{\sin (\xi-n \xi / k)}{\sin (\xi / k)} \cos n\left(\tau-\tau_{0}+\frac{\xi}{k}\right)\right] .
\end{aligned}
$$

Remark 14. According to (A.6) (see Appendices), it follows that coefficients (67) can be expressed as

$$
c_{n}=2 \sum_{m=1}^{k-n-1} m(k-n-m) \cos \left(\frac{(k-n-2 m) \xi}{k}\right) .
$$

Furthermore, from (74) it follows that coefficients $c_{k-2}, c_{k-3}$, $c_{k-4}$ and $c_{k-5}$ are equal to

$$
\begin{aligned}
& c_{k-2}=2, \\
& c_{k-3}=8 \cos \left(\frac{\xi}{k}\right), \\
& c_{k-4}=8+12 \cos \left(\frac{2 \xi}{k}\right), \\
& c_{k-5}=24 \cos \left(\frac{\xi}{k}\right)+16 \cos \left(\frac{3 \xi}{k}\right) .
\end{aligned}
$$

For example, for $k=2$, (75) and (68) lead to $c_{0}=2$ and $\lambda_{2}=1 /(2+\cos \xi)$, respectively, which from (72) further imply that

$$
T_{2}(\tau)=\frac{2\left[\cos (\xi / 2)-\cos \left(\tau-\tau_{0}+\xi / 2\right)\right]^{2}}{[2+\cos \xi]} .
$$

Also for $k=3$, (75), (76), and (68) lead to $c_{1}=2, c_{0}=$ $8 \cos (\xi / 3)$, and $\lambda_{3}=[2(3 \cos (\xi / 3)+\cos \xi)]^{-1}$, respectively, which from (72) further imply that

$$
\begin{aligned}
T_{3}(\tau)= & \frac{2\left[\cos (\xi / 3)-\cos \left(\tau-\tau_{0}+\xi / 3\right)\right]^{2}}{[3 \cos (\xi / 3)+\cos \xi]} \\
& \cdot\left[2 \cos \left(\frac{\xi}{3}\right)+\cos \left(\tau-\tau_{0}+\frac{\xi}{3}\right)\right] .
\end{aligned}
$$

Remark 15. According to (A.5) (see Appendices), relation (68) can be rewritten as

$$
\lambda_{k}=\left[(k-1) \cos \xi+k \sum_{n=1}^{k-1} \cos \left(\frac{(k-2 n) \xi}{k}\right)\right]^{-1} .
$$

Clearly, amplitude $\lambda_{k}$ of $k$ th harmonic of nonnegative waveform of type (35) with exactly two zeros is even function of $\xi$. Since $\cos ((k-2 n) \xi / k), n=0, \ldots,(k-1)$, decreases with increase of $|\xi|$ on interval $0 \leq|\xi| \leq \pi$, it follows that $\lambda_{k}$ monotonically increases with increase of $|\xi|$. Right hand side of $(68)$ is equal to $1 /\left(k^{2}-1\right)$ for $\xi=0$ and to one for $|\xi|=\pi$. Therefore, for nonnegative waveforms of type (35) with exactly two zeros, the following relation holds:

$$
\frac{1}{k^{2}-1}<\lambda_{k}<1
$$

The left boundary in (82) corresponds to maximally flat nonnegative waveforms (see Remark 11). The right boundary in (82) corresponds to nonnegative waveforms with $k$ zeros (also see Remark 11).

Amplitude of $k$ th harmonic of nonnegative waveform of type (35) with two zeros, as a function of parameter $\xi$ for $k \leq$ 5, is presented in Figure 5.

Remark 16. Nonnegative waveform of type (35) with two zeros can be also expressed in the following form:

$$
\begin{aligned}
T_{k}(\tau)= & 1-\lambda_{k} \frac{k \sin \xi}{\sin (\xi / k)} \cos \left(\tau-\tau_{0}+\frac{\xi}{k}\right) \\
& +\lambda_{k} \cos \left(k\left(\tau-\tau_{0}\right)+\xi\right),
\end{aligned}
$$




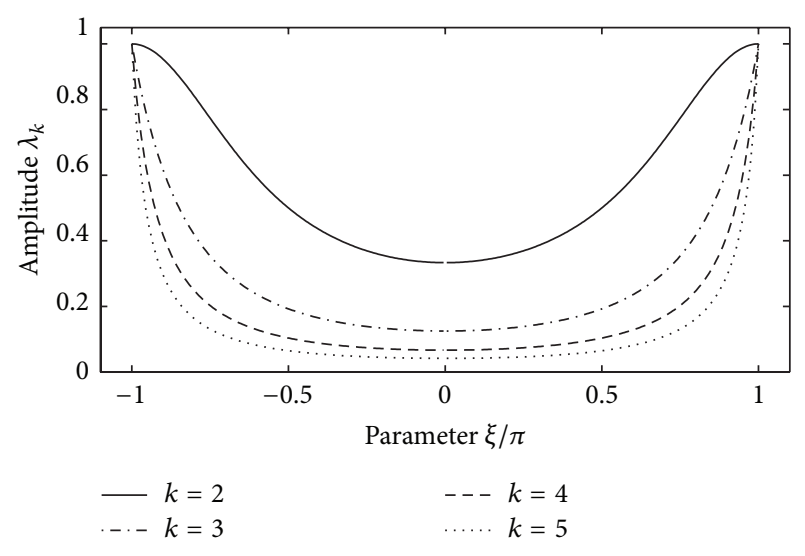

FIGURE 5: Amplitude of $k$ th harmonic of nonnegative waveform with two zeros as a function of parameter $\xi$.

where $\lambda_{k}$ is given by (68) and $0<|\xi|<\pi$. From (83) it follows that coefficients of fundamental harmonic of nonnegative waveform of type (35) with two zeros are

$$
a_{1}=-\lambda_{1} \cos \left(\tau_{0}-\frac{\xi}{k}\right), \quad b_{1}=-\lambda_{1} \sin \left(\tau_{0}-\frac{\xi}{k}\right),
$$

where $\lambda_{1}$ is amplitude of fundamental harmonic:

$$
\lambda_{1}=\frac{k \sin \xi}{\sin (\xi / k)} \lambda_{k}
$$

Coefficients of $k$ th harmonic are given by (45)-(46).

Notice that (68) can be rewritten as

$$
\lambda_{k}=\left[\cos \left(\frac{\xi}{k}\right) \frac{k \sin \xi}{\sin (\xi / k)}-\cos \xi\right]^{-1} .
$$

By introducing new variable,

$$
x=\cos \left(\frac{\xi}{k}\right),
$$

and using the Chebyshev polynomials (e.g., see Appendices), relations (85) and (86) can be rewritten as

$$
\begin{gathered}
\lambda_{1}=k \lambda_{k} U_{k-1}(x), \\
\lambda_{k}=\frac{1}{k x U_{k-1}(x)-V_{k}(x)},
\end{gathered}
$$

where $V_{k}(x)$ and $U_{k}(x)$ denote the Chebyshev polynomials of the first and second kind, respectively. From (89) it follows that

$$
\lambda_{k}\left[k x U_{k-1}(x)-V_{k}(x)\right]-1=0,
$$

which is polynomial equation of $k$ th degree in terms of variable $x$. From $0<|\xi|<\pi$ and (87) it follows that

$$
\cos \left(\frac{\pi}{k}\right)<x<1
$$

Since $\lambda_{k}$ is monotonically increasing function of $|\xi|, 0<|\xi|<$ $\pi$, it follows that $\lambda_{k}$ is monotonically decreasing function of $x$. This further implies that (90) has only one solution that satisfies (91). (For $k=2$ expression (91) $\operatorname{reads} \cos (\pi / 2) \leq$ $x<1$.) This solution for $x$ (which can be obtained at least numerically), according to (88), leads to amplitude $\lambda_{1}$ of fundamental harmonic.

For $k \leq 4$, solutions of (90) and (91) are

$$
\begin{gathered}
x=\sqrt{\frac{1-\lambda_{2}}{2 \lambda_{2}}}, \quad \frac{1}{3}<\lambda_{2} \leq 1, \\
x=\frac{1}{2 \sqrt[3]{\lambda_{3}}}, \quad \frac{1}{8}<\lambda_{3}<1, \\
x=\sqrt{\frac{1}{6}\left(1+\sqrt{\frac{5 \lambda_{4}+3}{2 \lambda_{4}}}\right)}, \quad \frac{1}{15}<\lambda_{4}<1 .
\end{gathered}
$$

Insertion of (92) into (88) leads to the following relations between amplitude $\lambda_{1}$ of fundamental and amplitude $\lambda_{k}$ of $k$ th harmonic, $k \leq 4$ :

$$
\begin{gathered}
\lambda_{1}=\sqrt{8 \lambda_{2}\left(1-\lambda_{2}\right)}, \quad \frac{1}{3}<\lambda_{2} \leq 1, \\
\lambda_{1}=3\left(\sqrt[3]{\lambda_{3}}-\lambda_{3}\right), \quad \frac{1}{8}<\lambda_{3}<1, \\
\lambda_{1}=\sqrt{\frac{32}{27}\left(\sqrt{2 \lambda_{4}\left(3+5 \lambda_{4}\right)^{3}}-2 \lambda_{4}\left(9+7 \lambda_{4}\right)\right),} \\
\frac{1}{15}<\lambda_{4}<1 .
\end{gathered}
$$

Proof of Proposition 9. As it has been shown earlier (see Proposition 6), nonnegative waveform of type (35) with at least one zero can be represented in form (38). Since we exclude nonnegative waveforms with $\lambda_{k}=1$, according to Remark 7, it follows that we exclude case $|\xi|=\pi$. Therefore in the quest for nonnegative waveforms of type (35) having two zeros we will start with waveforms of type (38) for $|\xi|<\pi$. It is clear that nonnegative waveforms of type (38) have two zeros if and only if

$$
\lambda_{k}=\left[\max _{\tau} r_{k}(\tau)\right]^{-1}
$$

and $\max _{\tau} r_{k}(\tau) \neq r_{k}\left(\tau_{0}\right)$. According to (64), $\max _{\tau} r_{k}(\tau) \neq$ $r_{k}\left(\tau_{0}\right)$ implies $|\xi| \neq 0$. Therefore, it is sufficient to consider only the interval (69).

Substituting (96) into (38) we obtain

$$
T_{k}(\tau)=\frac{\left[1-\cos \left(\tau-\tau_{0}\right)\right]\left[\max _{\tau} r_{k}(\tau)-r_{k}(\tau)\right]}{\max _{\tau} r_{k}(\tau)}
$$


Expression $\max _{\tau} r_{k}(\tau)-r_{k}(\tau)$, according to (64) and (39), equals

$$
\begin{aligned}
\max _{\tau} r_{k}(\tau)-r_{k}(\tau)= & k \frac{\sin ((k-1) \xi / k)}{\sin (\xi / k)} \\
& -2 \sum_{n=1}^{k-1}(k-n) \cos \left(n\left(\tau-\tau_{0}\right)+\xi\right) .
\end{aligned}
$$

Comparison of (97) with (66) yields

$$
\begin{aligned}
\max _{\tau} r_{k}(\tau)-r_{k}(\tau)= & {\left[1-\cos \left(\tau-\tau_{0}+\frac{2 \xi}{k}\right)\right] } \\
& \cdot\left[c_{0}+2 \sum_{n=1}^{k-2} c_{n} \cos n\left(\tau-\tau_{0}+\frac{\xi}{k}\right)\right],
\end{aligned}
$$

where coefficients $c_{n}, n=0, \ldots, k-2$, are given by (67). In what follows we are going to show that right hand sides of (98) and (99) are equal.

From (67) it follows that

$$
c_{0}-c_{1} \cos \left(\frac{\xi}{k}\right)=k \frac{\sin (\xi-\xi / k)}{\sin (\xi / k)} .
$$

Also, from (67) for $n=1, \ldots, k-3$ it follows that the following relations hold:

$$
\begin{gathered}
\left(c_{n-1}+c_{n+1}\right) \cos \left(\frac{\xi}{k}\right)-2 c_{n}=2(k-n) \cos \left(\xi-\frac{n \xi}{k}\right) \\
\left(c_{n-1}-c_{n+1}\right) \sin \left(\frac{\xi}{k}\right)=2(k-n) \sin \left(\xi-\frac{n \xi}{k}\right) .
\end{gathered}
$$

From (99), by using (75), (76), (100)-(101), and trigonometric identities

$$
\begin{aligned}
& \cos \left(\tau-\tau_{0}+\frac{2 \xi}{k}\right)= \cos \left(\frac{\xi}{k}\right) \cos \left(\tau-\tau_{0}+\frac{\xi}{k}\right) \\
&-\sin \left(\frac{\xi}{k}\right) \sin \left(\tau-\tau_{0}+\frac{\xi}{k}\right), \\
& \cos \left(\xi-\frac{n \xi}{k}\right) \cos \left(n\left(\tau-\tau_{0}+\frac{\xi}{k}\right)\right) \\
& \quad-\sin \left(\xi-\frac{n \xi}{k}\right) \sin \left(n\left(\tau-\tau_{0}+\frac{\xi}{k}\right)\right) \\
&=\cos \left(n\left(\tau-\tau_{0}\right)+\xi\right),
\end{aligned}
$$

we obtain (98). Consequently (98) and (99) are equal, which completes the proof.

\subsection{Nonnegative Waveforms with Two Zeros and Prescribed} Coefficients ofkth Harmonic. In this subsection we show that, for prescribed coefficients $a_{k}$ and $b_{k}$, there are $k$ nonnegative waveforms of type (35) with exactly two zeros. According to
(37) and (82), coefficients $a_{k}$ and $b_{k}$ of nonnegative waveforms of type (35) with exactly two zeros satisfy the following relation:

$$
\frac{1}{k^{2}-1}<\sqrt{a_{k}^{2}+b_{k}^{2}}<1
$$

According to Remark 16, the value of $x$ (see (87)) that corresponds to $\lambda_{k}=\sqrt{a_{k}^{2}+b_{k}^{2}}$ can be determined from (90)(91). As we mentioned earlier, (90) has only one solution that satisfies (91). This value of $x$, according to (88), leads to the amplitude $\lambda_{1}$ of fundamental harmonic (closed form expressions for $\lambda_{1}$ in terms of $\lambda_{k}$ and $k \leq 4$ are given by (93)(95)).

On the other hand, from (45)-(46) it follows that

$$
k \tau_{0}-\xi=\operatorname{atan} 2\left(b_{k}, a_{k}\right)+2 q \pi, \quad q=1, \ldots,(k-1),
$$

where function $\operatorname{atan} 2(y, x)$ is defined as

$$
\operatorname{atan} 2(y, x)= \begin{cases}\arctan \left(\frac{y}{x}\right) & \text { if } x \geq 0, \\ \arctan \left(\frac{y}{x}\right)+\pi & \text { if } x<0, y \geq 0, \\ \arctan \left(\frac{y}{x}\right)-\pi & \text { if } x<0, y<0,\end{cases}
$$

with the codomain $(-\pi, \pi]$. Furthermore, according to (84) and (104), the coefficients of fundamental harmonic of nonnegative waveforms with two zeros and prescribed coefficients of $k$ th harmonic are equal to

$$
\begin{aligned}
& a_{1}=-\lambda_{1} \cos \left[\frac{\operatorname{atan} 2\left(b_{k}, a_{k}\right)+2 q \pi}{k}\right], \\
& b_{1}=-\lambda_{1} \sin \left[\frac{\operatorname{atan} 2\left(b_{k}, a_{k}\right)+2 q \pi}{k}\right],
\end{aligned}
$$

where $q=0, \ldots,(k-1)$. For chosen $q$, according to (104) and (66), positions of zeros are

$$
\begin{gathered}
\tau_{0}=\frac{1}{k}\left[\xi+\operatorname{atan} 2\left(b_{k}, a_{k}\right)+2 q \pi\right], \\
\tau_{0}-\frac{2 \xi}{k}=\frac{1}{k}\left[-\xi+\operatorname{atan} 2\left(b_{k}, a_{k}\right)+2 q \pi\right] .
\end{gathered}
$$

From $(106)$ and $q=0, \ldots,(k-1)$ it follows that, for prescribed coefficients $a_{k}$ and $b_{k}$, there are $k$ nonnegative waveforms of type (35) with exactly two zeros.

We provide here an algorithm to facilitate calculation of coefficients $a_{1}$ and $b_{1}$ of nonnegative waveforms of type (35) with two zeros and prescribed coefficients $a_{k}$ and $b_{k}$, providing that $a_{k}$ and $b_{k}$ satisfy (103). 


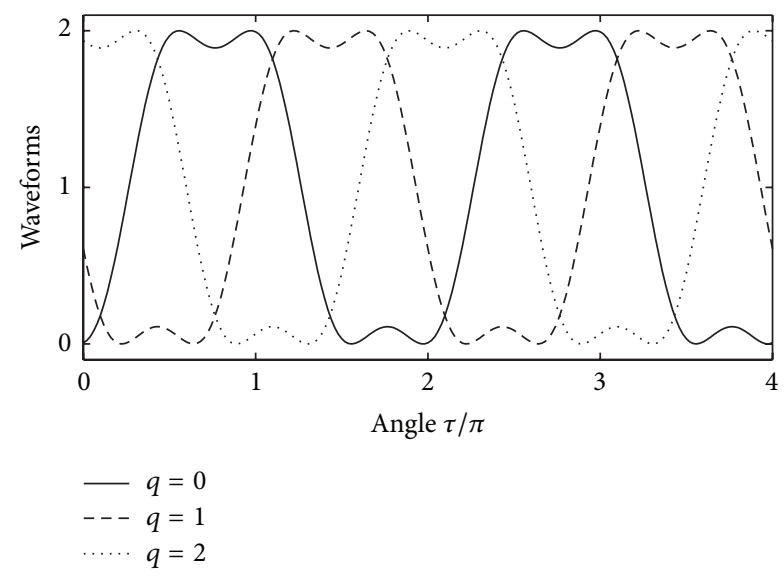

FIGURE 6: Nonnegative waveforms with two zeros for $k=3, a_{3}=$ -0.15 , and $b_{3}=-0.2$.

Algorithm 17. (i) Calculate $\lambda_{k}=\sqrt{a_{k}^{2}+b_{k}^{2}}$,

(ii) identify $x$ that satisfies both relations (90) and (91),

(iii) calculate $\lambda_{1}$ according to (88),

(iv) choose integer $q$, such that $0 \leq q \leq k-1$,

(v) calculate $a_{1}$ and $b_{1}$ according to (106).

For $k \leq 4$, by using (93) for $k=2$, (94) for $k=3$, and (95) for $k=4$ it is possible to calculate directly $\lambda_{1}$ from $\lambda_{k}$ and proceed to step (iv).

For $k=2$ and prescribed coefficients $a_{2}$ and $b_{2}$, there are two waveforms with two zeros, one corresponding to $a_{1}<0$ and the other corresponding to $a_{1}>0$ (see also [12]).

Let us take as an input $k=3, a_{3}=-0.15$, and $b_{3}=-0.2$. Execution of Algorithm 17 on this input yields $\lambda_{3}=0.25$ and $\lambda_{1}=1.1399$ (according to (94)). For $q=0$ we calculate $a_{1}=-0.8432$ and $b_{1}=0.7670$ (corresponding waveform is presented by solid line in Figure 6); for $q=1$ we calculate $a_{1}=-0.2426$ and $b_{1}=-1.1138$ (corresponding waveform is presented by dashed line); for $q=2$ we calculate $a_{1}=1.0859$ and $b_{1}=0.3468$ (corresponding waveform is presented by dotted line).

As another example of the usage of Algorithm 17, let us consider case $k=4$ and assume that $a_{4}=-0.15$ and $b_{4}=-0.2$. Consequently $\lambda_{4}=0.25$ and $\lambda_{1}=0.9861$ (according to (95)). For $q=0, \ldots, 3$ we calculate the following four pairs $\left(a_{1}, b_{1}\right)$ of coefficients of fundamental harmonic: $(-0.8388,0.5184)$ for $q=0,(-0.5184,-0.8388)$ for $q=1,(0.8388,-0.5184)$ for $q=$ 2 , and $(0.5184,0.8388)$ for $q=3$. Corresponding waveforms are presented in Figure 7.

\section{Nonnegative Waveforms with Maximal Amplitude of Fundamental Harmonic}

In this section we provide general description of nonnegative waveforms containing fundamental and $k$ th harmonic with maximal amplitude of fundamental harmonic for prescribed amplitude of $k$ th harmonic.

The main result of this section is presented in the following proposition.

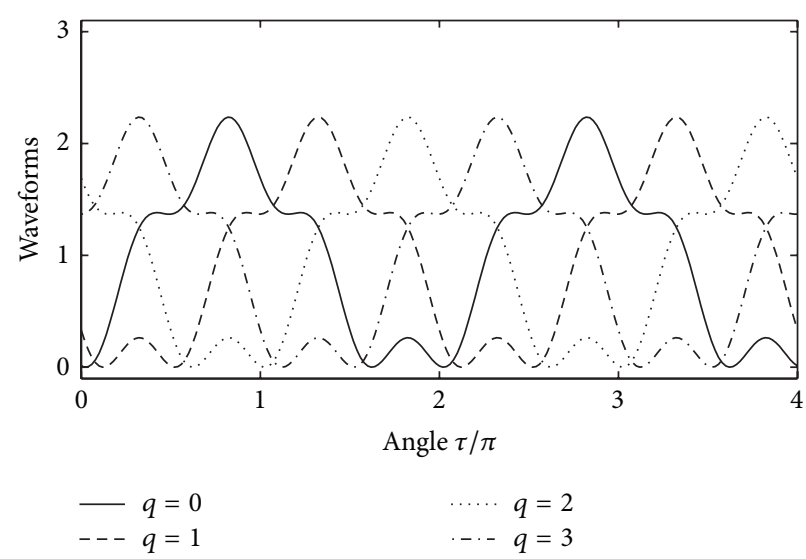

FIGURE 7: Nonnegative waveforms with two zeros for $k=4, a_{4}=$ -0.15 , and $b_{4}=-0.2$.

Proposition 18. Every nonnegative waveform of type (35) with maximal amplitude $\lambda_{1}$ of fundamental harmonic and prescribed amplitude $\lambda_{k}$ of $k$ th harmonic can be expressed in the following form:

$$
\begin{aligned}
T_{k}(\tau)= & {\left[1-\cos \left(\tau-\tau_{0}\right)\right] } \\
& \cdot\left[1-(k-1) \lambda_{k}-2 \lambda_{k} \sum_{n=1}^{k-1}(k-n) \cos \left(n\left(\tau-\tau_{0}\right)\right)\right],
\end{aligned}
$$

if $0 \leq \lambda_{k} \leq 1 /\left(k^{2}-1\right)$ or

$$
\begin{aligned}
T_{k}(\tau)= & \lambda_{k}\left[1-\cos \left(\tau-\tau_{0}\right)\right]\left[1-\cos \left(\tau-\tau_{0}+\frac{2 \xi}{k}\right)\right] \\
& \cdot\left[c_{0}+2 \sum_{n=1}^{k-2} c_{n} \cos n\left(\tau-\tau_{0}+\frac{\xi}{k}\right)\right]
\end{aligned}
$$

if $1 /\left(k^{2}-1\right) \leq \lambda_{k} \leq 1$, providing that $c_{n}, n=0, \ldots, k-2$, and $\lambda_{k}$ are related to $\xi$ via relations (67) and (68), respectively, and $|\xi| \leq \pi$.

Remark 19. Expression (108) can be obtained from (38) by setting $\xi=0$. Furthermore, insertion of $\xi=0$ into (43)-(46) leads to the following expressions for coefficients of waveform of type (108):

$$
\begin{array}{cc}
a_{1}=-\left(1+\lambda_{k}\right) \cos \tau_{0}, & b_{1}=-\left(1+\lambda_{k}\right) \sin \tau_{0}, \\
a_{k}=\lambda_{k} \cos \left(k \tau_{0}\right), & b_{k}=\lambda_{k} \sin \left(k \tau_{0}\right) .
\end{array}
$$

On the other hand, (109) coincides with (66). Therefore, the expressions for coefficients of (109) and (66) also coincide. Thus, expressions for coefficients of fundamental harmonic of waveform (109) are given by (84), where $\lambda_{1}$ is given by (85), while expressions for coefficients of $k$ th harmonic are given by (45)-(46).

Waveforms described by (108) have exactly one zero, while waveforms described by (109) for $1 /\left(k^{2}-1\right)<\lambda_{k}<1$ 


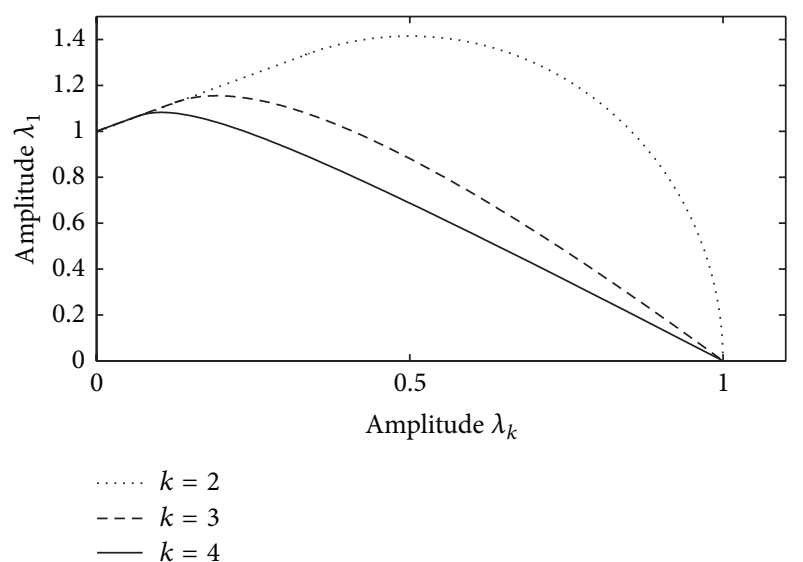

FIGURE 8: Maximal amplitude of fundamental harmonic as a function of amplitude of $k$ th harmonic.

have exactly two zeros. As we mentioned earlier, waveforms (109) for $\lambda_{k}=1$ have $k$ zeros.

Remark 20. Maximal amplitude of fundamental harmonic of nonnegative waveforms of type (35) for prescribed amplitude of $k$ th harmonic can be expressed as

$$
\lambda_{1}=1+\lambda_{k}
$$

if $0 \leq \lambda_{k} \leq 1 /\left(k^{2}-1\right)$, or

$$
\lambda_{1}=\frac{k \sin \xi}{k \sin \xi \cos (\xi / k)-\cos \xi \sin (\xi / k)},
$$

if $1 /\left(k^{2}-1\right) \leq \lambda_{k} \leq 1$, where $\xi$ is related to $\lambda_{k}$ via (68) (or (86)) and $|\xi| \leq \pi$.

From (110) it follows that (111) holds. Substitution of (86) into (85) leads to (112).

Notice that $\lambda_{k}=1 /\left(k^{2}-1\right)$ is the only common point of the intervals $0 \leq \lambda_{k} \leq 1 /\left(k^{2}-1\right)$ and $1 /\left(k^{2}-1\right) \leq \lambda_{k} \leq$ 1. According to (111), $\lambda_{k}=1 /\left(k^{2}-1\right)$ corresponds to $\lambda_{1}=$ $k^{2} /\left(k^{2}-1\right)$. It can be also obtained from (112) by setting $\xi=0$. The waveforms corresponding to this pair of amplitudes are maximally flat nonnegative waveforms.

Maximal amplitude of fundamental harmonic of nonnegative waveform of type (35) for $k \leq 4$, as a function of amplitude of $k$ th harmonic, is presented in Figure 8.

Remark 21. Maximum value of amplitude of fundamental harmonic of nonnegative waveform of type (35) is

$$
\lambda_{1, \max }=\frac{1}{\cos (\pi /(2 k))} .
$$

This maximum value is attained for $|\xi|=\pi / 2$ (see (112)). The corresponding value of amplitude of $k$ th harmonic is $\lambda_{k}=$ $(1 / k) \tan (\pi /(2 k))$. Nonnegative waveforms of type (35) with $\lambda_{1}=\lambda_{1, \max }$ have two zeros at $\tau_{0}$ and $\tau_{0}-\pi / k$ for $\xi=\pi / 2$, or at $\tau_{0}$ and $\tau_{0}+\pi / k$ for $\xi=-\pi / 2$.

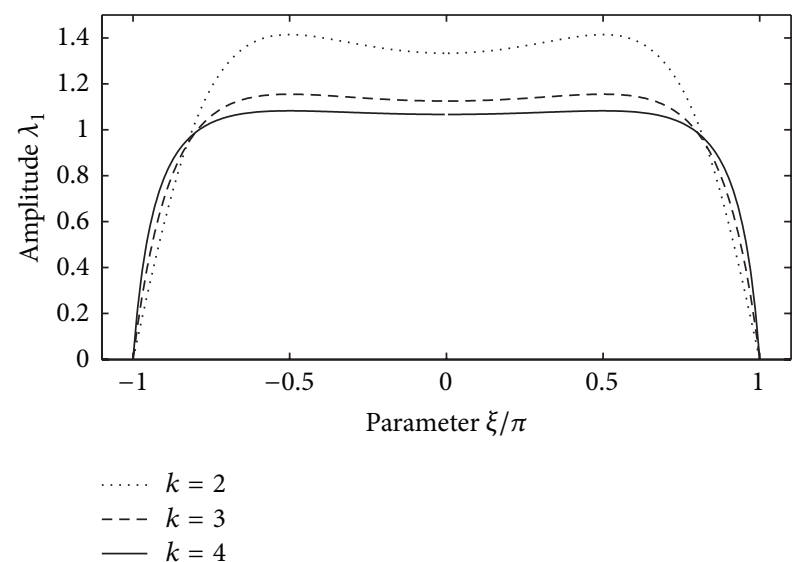

FIGURE 9: Maximal amplitude of fundamental harmonic as a function of parameter $\xi$.

To prove that (113) holds, let us first show that the following relation holds for $k \geq 2$ :

$$
\cos \left(\frac{\pi}{2 k}\right)<1-\frac{1}{k^{2}} \text {. }
$$

From $k \geq 2$, it follows that $\operatorname{sinc}(\pi /(4 k))>\operatorname{sinc}(\pi / 4)$, where $\operatorname{sinc} x=(\sin x) / x$, and therefore $\sin (\pi /(4 k))>1 /(\sqrt{2} k)$. By using trigonometric identity $\cos 2 x=1-2 \sin ^{2} x$, we immediately obtain (114).

According to (111) and (112), it is clear that $\lambda_{1}$ attains its maximum value on the interval $1 /\left(k^{2}-1\right) \leq \lambda_{k} \leq 1$. Since $\lambda_{k}$ is monotonic function of $|\xi|$ on interval $|\xi| \leq \pi$ (see Remark 15), it follows that $d \lambda_{k} / d \xi \neq 0$ for $0<|\xi|<\pi$. Therefore, to find critical points of $\lambda_{1}$ as a function of $\lambda_{k}$ it is sufficient to find critical points of $\lambda_{1}$ as a function of $|\xi|, 0<|\xi|<\pi$, and consider its values at the end points $\xi=0$ and $|\xi|=\pi$. Plot of $\lambda_{1}$ as a function of parameter $\xi$ for $k \leq 4$ is presented in Figure 9. According to (112), first derivative of $\lambda_{1}$ with respect to $\xi$ is equal to zero if and only if $(k \cos \xi \sin (\xi / k)-\sin \xi \cos (\xi / k)) \cos \xi=0$. On interval $0<|\xi|<\pi$, this is true if and only if $|\xi|=\pi / 2$. According to (112), $\lambda_{1}$ is equal to $k^{2} /\left(k^{2}-1\right)$ for $\xi=0$, equal to zero for $|\xi|=\pi$, and equal to $1 / \cos (\pi /(2 k))$ for $|\xi|=\pi / 2$. From (114) it follows that $k^{2} /\left(k^{2}-1\right)<1 / \cos (\pi /(2 k))$ and therefore maximum value of $\lambda_{1}$ is given by (113). Moreover, maximum value of $\lambda_{1}$ is attained for $|\xi|=\pi / 2$.

According to above consideration, all nonnegative waveforms of type (35) having maximum value of amplitude of fundamental harmonic can be obtained from (109) by setting $|\xi|=\pi / 2$. Three of them corresponding to $k=3, \xi=\pi / 2$, and three different values of $\tau_{0}(0, \pi / 6$, and $\pi / 3)$ are presented in Figure 10. Dotted line corresponds to $\tau_{0}=0$ (coefficients of corresponding waveform are $a_{1}=-1, b_{1}=1 / \sqrt{3}, a_{3}=0$, and $\left.b_{3}=-\sqrt{3} / 9\right)$, solid line to $\tau_{0}=\pi / 6\left(a_{1}=-2 / \sqrt{3}, b_{1}=0\right.$, $a_{3}=\sqrt{3} / 9$, and $\left.b_{3}=0\right)$, and dashed line to $\tau_{0}=\pi / 3\left(a_{1}=-1\right.$, $b_{1}=-1 / \sqrt{3}, a_{3}=0$, and $\left.b_{3}=\sqrt{3} / 9\right)$.

Proof of Proposition 18. As it has been shown earlier (Proposition 6), nonnegative waveform of type (35) with at least 


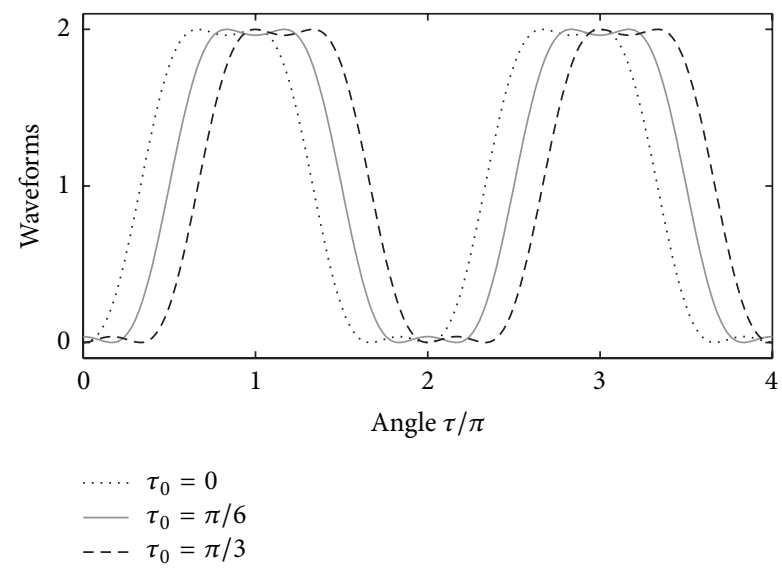

FIGURE 10: Nonnegative waveforms with maximum amplitude of fundamental harmonic for $k=3$ and $\xi=\pi / 2$.

one zero can be represented in form (38). According to (43), (44), and (36), for amplitude $\lambda_{1}$ of fundamental harmonic of waveforms of type (38) the following relation holds:

$$
\lambda_{1}=\sqrt{\left(1+\lambda_{k} \cos \xi\right)^{2}+k^{2} \lambda_{k}^{2} \sin ^{2} \xi}
$$

where $\lambda_{k}$ satisfy (40) and $|\xi| \leq \pi$.

Because of (40), in the quest of finding maximal $\lambda_{1}$ for prescribed $\lambda_{k}$, we have to consider the following two cases:

$$
\begin{aligned}
& \text { (Case i) } \lambda_{k}<[(k-1) \cos \xi+k \sin (\xi-\xi / k) / \sin (\xi / k)]^{-1} . \\
& \text { (Case ii) } \lambda_{k}=[(k-1) \cos \xi+k \sin (\xi-\xi / k) / \sin (\xi / k)]^{-1} .
\end{aligned}
$$

Case i. Since $\lambda_{k}<[(k-1) \cos \xi+k \sin (\xi-\xi / k) / \sin (\xi / k)]^{-1}$ implies $\lambda_{k} \neq 1$, according to (115), it follows that $\lambda_{1} \neq 0$. Hence $d \lambda_{1} / d \xi=0$ implies

$$
2 \lambda_{k} \sin \xi\left[1-\left(k^{2}-1\right) \lambda_{k} \cos \xi\right]=0 .
$$

Therefore $d \lambda_{1} / d \xi=0$ if $\lambda_{k}=0$ (Option 1) or $\sin \xi=0$ (Option 2) or $\left(k^{2}-1\right) \lambda_{k} \cos \xi=1$ (Option 3).

Option 1. According to (115), $\lambda_{k}=0$ implies $\lambda_{1}=1$ (notice that this implication shows that $\lambda_{1}$ does not depend on $\xi$ and therefore we can set $\xi$ to zero value).

Option 2. According to (115), $\sin \xi=0$ implies $\lambda_{1}=1+$ $\lambda_{k} \cos \xi$, which further leads to the conclusion that $\lambda_{1}$ is maximal for $\xi=0$. For $\xi=0, \lambda_{k}<[(k-1) \cos \xi+k \sin (\xi-$ $\xi / k) / \sin (\xi / k)]^{-1}$ becomes $\lambda_{k}<1 /\left(k^{2}-1\right)$.

Option 3. This option leads to contradiction. To show that, notice that $\left(k^{2}-1\right) \lambda_{k} \cos \xi=1$ and $\lambda_{k}<[(k-$ 1) $\cos \xi+k \sin (\xi-\xi / k) / \sin (\xi / k)]^{-1}$ imply that $(k-1) \cos \xi>$ $\sin (\xi-\xi / k) / \sin (\xi / k)$. Using (A.5) (see Appendices), the latest inequality can be rewritten as $\sum_{n=1}^{k-1}[\cos \xi-\cos ((k-2 n) \xi / k)]>$ 0 . But, from $|k-2 n|<k, n=1, \ldots,(k-1)$, and $|\xi| \leq \pi$ it follows that all summands are not positive and therefore $(k-1) \cos \xi>\sin (\xi-\xi / k) / \sin (\xi / k)$ does not hold for $|\xi| \leq \pi$.
Consequently, Case i implies $\xi=0$ and $\lambda_{k}<1 /\left(k^{2}-1\right)$. Finally, substitution of $\xi=0$ into (38) leads to (108), which proves that (108) holds for $\lambda_{k}<1 /\left(k^{2}-1\right)$.

Case ii. Relation $\lambda_{k}=[(k-1) \cos \xi+k \sin (\xi-\xi / k) / \sin (\xi / k)]^{-1}$, according to Proposition 9 and Remark 11, implies that corresponding waveforms can be expressed via (66)-(68) for $|\xi| \leq \pi$. Furthermore, $\lambda_{k}=[(k-1) \cos \xi+k \sin (\xi-$ $\xi / k) / \sin (\xi / k)]^{-1}$ and $|\xi| \leq \pi$ imply $1 /\left(k^{2}-1\right) \leq \lambda_{k} \leq 1$. This proves that (109) holds for $1 /\left(k^{2}-1\right) \leq \lambda_{k} \leq 1$.

Finally, let us prove that (108) holds for $\lambda_{k}=1 /\left(k^{2}-\right.$ 1). According to (68) (see also Remark 11), this value of $\lambda_{k}$ corresponds to $\xi=0$. Furthermore, substitution of $\lambda_{k}=$ $1 /\left(k^{2}-1\right)$ and $\xi=0$ into (109) leads to (70), which can be rewritten as

$$
\begin{aligned}
T_{k}(\tau)= & \frac{\left[1-\cos \left(\tau-\tau_{0}\right)\right]}{\left(1-k^{2}\right)} \\
& \cdot\left[k(k-1)-2 \sum_{n=1}^{k-1}(k-n) \cos \left(n\left(\tau-\tau_{0}\right)\right)\right] .
\end{aligned}
$$

Waveform (117) coincides with waveform (108) for $\lambda_{k}=$ $1 /\left(1-k^{2}\right)$. Consequently, (108) holds for $\lambda_{k}=1 /\left(1-k^{2}\right)$, which completes the proof.

\section{Nonnegative Waveforms with Maximal Absolute Value of the Coefficient of Cosine Term of Fundamental Harmonic}

In this section we consider general description of nonnegative waveforms of type (35) with maximal absolute value of coefficient $a_{1}$ for prescribed coefficients of $k$ th harmonic. This type of waveform is of particular interest in PA efficiency analysis. In a number of cases of practical interest either current or voltage waveform is prescribed. In such cases, the problem of finding maximal efficiency of PA can be reduced to the problem of finding nonnegative waveform with maximal coefficient $a_{1}$ for prescribed coefficients of $k$ th harmonic (see also Section 7).

In Section 5.1 we provide general description of nonnegative waveforms of type (35) with maximal absolute value of coefficient $a_{1}$ for prescribed coefficients of $k$ th harmonic. In Section 5.2 we illustrate results of Section 5.1 for particular case $k=3$.

5.1. Nonnegative Waveforms with Maximal Absolute Value of Coefficient $a_{1}$ for $k \geq 2$. Waveforms $T_{k}(\tau)$ of type (35) with $a_{1} \geq 0$ can be derived from those with $a_{1} \leq 0$ by shifting by $\pi$, and therefore we can assume without loss of generality that $a_{1} \leq 0$. Notice that if $k$ is even, then shifting $T_{k}(\tau)$ by $\pi$ produces the same result as replacement of $a_{1}$ with $-a_{1}$ ( $a_{k}$ remains the same). On the other hand, if $k$ is odd, then shifting $T_{k}(\tau)$ by $\pi$ produces the same result as replacement of $a_{1}$ with $-a_{1}$ and $a_{k}$ with $-a_{k}$.

According to (37), coefficients of $k$ th harmonic can be expressed as

$$
a_{k}=\lambda_{k} \cos \delta, \quad b_{k}=\lambda_{k} \sin \delta,
$$


where

$$
|\delta| \leq \pi .
$$

Conversely, for prescribed coefficients $a_{k}$ and $b_{k}, \delta$ can be determined as

$$
\delta=\operatorname{atan} 2\left(b_{k}, a_{k}\right),
$$

where definition of function atan $2(y, x)$ is given by (105).

The main result of this section is stated in the following proposition.

Proposition 22. Every nonnegative waveform of type (35) with maximal absolute value of coefficient $a_{1} \leq 0$ for prescribed coefficients $a_{k}$ and $b_{k}$ of $k$ th harmonic can be represented as

$$
\begin{aligned}
T_{k}(\tau) & \\
= & {[1-\cos \tau] } \\
& \cdot\left[1-(k-1) a_{k}-2 \sum_{n=1}^{k-1}(k-n)\left(a_{k} \cos n \tau+b_{k} \sin n \tau\right)\right],
\end{aligned}
$$

if $k \lambda_{k}[\sin \delta / \sin (\delta / k)] \cos (\delta / k) \leq 1+a_{k}$, where $\delta=\operatorname{atan} 2\left(b_{\mathrm{k}}\right.$, $\left.a_{k}\right)$, or

$$
\begin{aligned}
T_{k}(\tau)=\lambda_{k} & {\left[1-\cos \left(\tau-\frac{(\delta+\xi)}{k}\right)\right] } \\
& \cdot\left[1-\cos \left(\tau-\frac{(\delta-\xi)}{k}\right)\right] \\
& \cdot\left[c_{0}+2 \sum_{n=1}^{k-2} c_{n} \cos n\left(\tau-\frac{\delta}{k}\right)\right],
\end{aligned}
$$

if $k \lambda_{k}[\sin \delta / \sin (\delta / k)] \cos (\delta / k) \geq 1+a_{k}$, where $c_{n}, n=0, \ldots$, $k-2$, and $\lambda_{k}=\sqrt{a_{k}^{2}+b_{k}^{2}}$ are related to $\xi$ via relations (67) and (68), respectively, and $|\xi| \leq \pi$.

Remark 23. Expression (121) can be obtained from (38) by setting $\tau_{0}=0$ and $\xi=-\delta$ and then replacing $\lambda_{k} \cos \delta$ with $a_{k}$ (see (118)) and $\lambda_{k} \cos (n \tau-\delta)$ with $a_{k} \cos n \tau+b_{k} \sin n \tau$ (see also (118)). Furthermore, insertion of $\tau_{0}=0$ and $\xi=$ $-\delta$ into (43)-(46) leads to the following relations between fundamental and $k$ th harmonic coefficients of waveform (121):

$$
a_{1}=-\left(1+a_{k}\right), \quad b_{1}=-k b_{k} .
$$

On the other hand, expression (122) can be obtained from (66) by replacing $\tau_{0}-\xi / k$ with $\delta / k$. Therefore, substitution of $\tau_{0}-\xi / k=\delta / k$ in (84) leads to

$$
a_{1}=-\lambda_{1} \cos \left(\frac{\delta}{k}\right), \quad b_{1}=-\lambda_{1} \sin \left(\frac{\delta}{k}\right),
$$

where $\lambda_{1}$ is given by (85).

The fundamental harmonic coefficients $a_{1}$ and $b_{1}$ of waveform of type (35) with maximal absolute value of coefficient $a_{1} \leq 0$ satisfy both relations (123) and (124) if $a_{k}$ and $b_{k}$ satisfy $1+a_{k}=k \lambda_{k}[\sin \delta / \sin (\delta / k)] \cos (\delta / k)$. For such waveforms, relations $\tau_{0}=0$ and $\xi=-\delta$ also hold.
Remark 24. Amplitude of $k$ th harmonic of nonnegative waveform of type (35) with maximal absolute value of coefficient $a_{1} \leq 0$ and coefficients $a_{k}, b_{k}$ satisfying $1+a_{k}=$ $k \lambda_{k}[\sin \delta / \sin (\delta / k)] \cos (\delta / k)$, is

$$
\lambda_{k}=\frac{\sin (\delta / k)}{k \sin \delta \cos (\delta / k)-\cos \delta \sin (\delta / k)} .
$$

To show that, it is sufficient to substitute $a_{k}=\lambda_{k} \cos \delta$ (see (118)) into $1+a_{k}=k \lambda_{k}[\sin \delta / \sin (\delta / k)] \cos (\delta / k)$.

Introducing new variable,

$$
y=\cos \left(\frac{\delta}{k}\right),
$$

and using the Chebyshev polynomials (e.g., see Appendices), relations $a_{k}=\lambda_{k} \cos \delta$ and (125) can be rewritten as

$$
\begin{gathered}
a_{k}=\lambda_{k} V_{k}(y), \\
\lambda_{k}=\frac{1}{k y U_{k-1}(y)-V_{k}(y)},
\end{gathered}
$$

where $V_{k}(y)$ and $U_{k}(y)$ denote the Chebyshev polynomials of the first and second kind, respectively. Substitution of (128) into (127) leads to

$$
a_{k} k y U_{k-1}(y)-\left(1+a_{k}\right) V_{k}(y)=0,
$$

which is polynomial equation of $k$ th degree in terms of variable $y$. From $|\delta| \leq \pi$ and (126) it follows that

$$
\cos \left(\frac{\pi}{k}\right) \leq y \leq 1 .
$$

In what follows we show that $a_{k}$ is monotonically increasing function of $y$ on the interval (130). From $\xi=-\delta$ (see Remark 23) and (81) it follows that $\lambda_{k}^{-1}=(k-1) \cos \delta+$ $k \sum_{n=1}^{k-1} \cos ((k-2 n) \delta / k) \geq 1$ and therefore $a_{k}=\lambda_{k} \cos \delta$ can be rewritten as

$$
a_{k}=\frac{\cos \delta}{(k-1) \cos \delta+k \sum_{n=1}^{k-1} \cos ((k-2 n) \delta / k)} .
$$

Obviously $a_{k}$ is even function of $\delta$ and all cosines in (131) are monotonically decreasing functions of $|\delta|$ on the interval $|\delta| \leq \pi$. It is easy to show that $\cos ((k-2 n) \delta / k), n=$ $1, \ldots,(k-1)$, decreases slower than $\cos \delta$ when $|\delta|$ increases. This implies that denominator of the right hand side of (131) decreases slower than numerator. Since denominator is positive for $|\delta| \leq \pi$ it further implies that $a_{k}$ is decreasing function of $|\delta|$ on interval $|\delta| \leq \pi$. Consequently, $a_{k}$ is monotonically increasing function of $y$ on the interval (130).

Thus we have shown that $a_{k}$ is monotonically increasing function of $y$ on the interval (130) and therefore (129) has only one solution that satisfies (130). According to (128), the value of $y$ obtained from (129) and (130), either analytically or numerically, leads to amplitude $\lambda_{k}$ of $k$ th harmonic. 


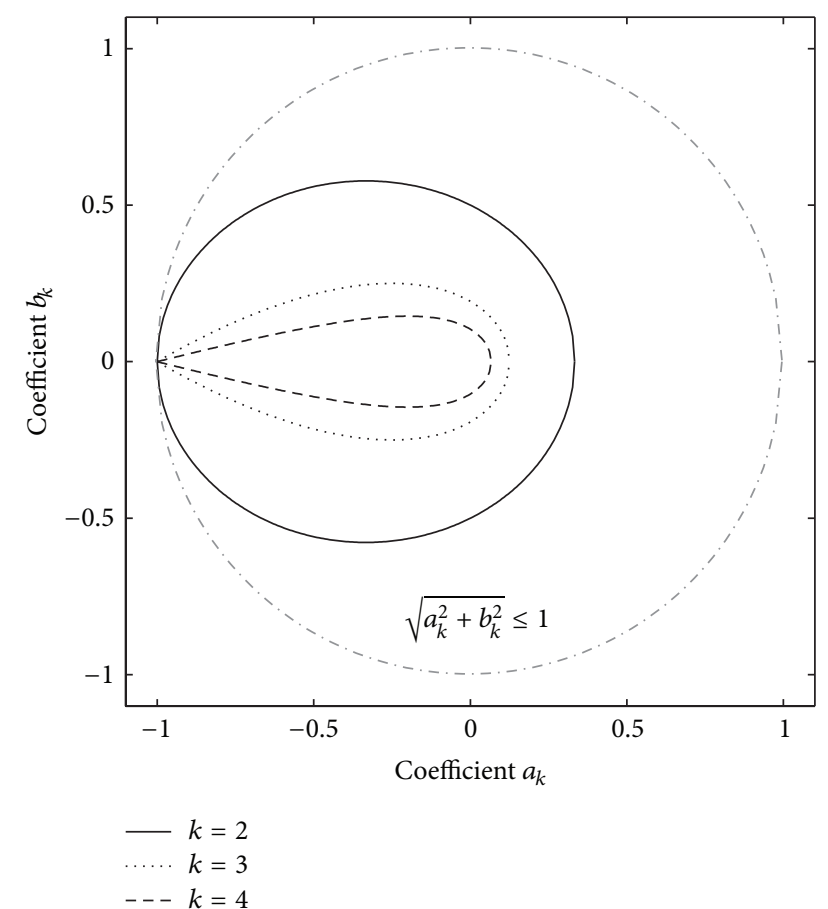

FIgURE 11: Plot of $\left(a_{k}, b_{k}\right)$ satisfying $1+a_{k}=k \lambda_{k}[\sin \delta / \sin (\delta /$ $k)] \cos (\delta / k)$ for $k \leq 4$.

By solving (129) and (130) for $k \leq 4$ we obtain

$$
\begin{gathered}
y=\sqrt{\frac{1+a_{2}}{2\left(1-a_{2}\right)},} \quad-1 \leq a_{2} \leq \frac{1}{3}, \\
y=\sqrt{\frac{3}{4\left(1-2 a_{3}\right)},} \quad-1 \leq a_{3} \leq \frac{1}{8}, \\
y=\sqrt{\frac{\sqrt{2-4 a_{4}+10 a_{4}^{2}}-2\left(1-a_{4}\right)}{4\left(1-3 a_{4}\right)}}, \quad-1 \leq a_{4} \leq \frac{1}{15} .
\end{gathered}
$$

Insertion of (132) into (128) leads to the following explicit expressions for the amplitude $\lambda_{k}, k \leq 4$ :

$$
\begin{gathered}
\lambda_{2}=\frac{1}{2}\left(1-a_{2}\right), \quad-1 \leq a_{2} \leq \frac{1}{3}, \\
\lambda_{3}^{2}=\left[\frac{1}{3}\left(1-2 a_{3}\right)\right]^{3}, \quad-1 \leq a_{3} \leq \frac{1}{8}, \\
\lambda_{4}=\frac{1}{4}\left(-1-a_{4}+\sqrt{2-4 a_{4}+10 a_{4}^{2}}\right), \quad-1 \leq a_{4} \leq \frac{1}{15} .
\end{gathered}
$$

Relations (133)-(135) define closed lines (see Figure 11) which separate points representing waveforms of type (121) from points representing waveforms of type (122). For given $k$, points inside the corresponding curve refer to nonnegative waveforms of type (121), whereas points outside curve (and $\left.\sqrt{a_{k}^{2}+b_{k}^{2}} \leq 1\right)$ correspond to nonnegative waveforms of type
(122). Points on the respective curve correspond to the waveforms which can be expressed in both forms (121) and (122).

Remark 25. The maximum absolute value of coefficient $a_{1}$ of nonnegative waveform of type (35) is

$$
\left|a_{1}\right|_{\max }=\frac{1}{\cos (\pi /(2 k))}
$$

This maximum value is attained for $|\xi|=\pi / 2$ and $\delta=0$ (see (124)). Notice that $\left|a_{1}\right|_{\max }$ is equal to the maximum value $\lambda_{1, \max }$ of amplitude of fundamental harmonic (see (113)). Coefficients of waveform with maximum absolute value of coefficient $a_{1}, a_{1}<0$, are

$$
\begin{gathered}
a_{1}=-\frac{1}{\cos (\pi /(2 k))}, \quad a_{k}=\frac{1}{k} \tan \left(\frac{\pi}{(2 k)}\right), \\
b_{1}=b_{k}=0 .
\end{gathered}
$$

Waveform described by (137) is cosine waveform having zeros at $\pi /(2 k)$ and $-\pi /(2 k)$.

In the course of proving (136), notice first that $\left|a_{1}\right|_{\max } \leq$ $\lambda_{1, \max }$ holds. According to (123) and (124), maximum of $\left|a_{1}\right|$ occurs for $k \lambda_{k}[\sin \delta / \sin (\delta / k)] \cos (\delta / k) \geq 1+a_{k}$. From (124) it immediately follows that maximum value of $\left|a_{1}\right|$ is attained if and only if $\lambda_{1}=\lambda_{1, \max }$ and $\delta=0$, which because of $\delta / k=\tau_{0}-\xi / k$ further implies $\tau_{0}=\xi / k$. Since maximum value of $\lambda_{1}$ is attained for $|\xi|=\pi / 2$, it follows that corresponding waveform has zeros at $\pi /(2 k)$ and $-\pi /(2 k)$.

Proof of Proposition 22. As it was mentioned earlier in this section, we can assume without loss of generality that $a_{1} \leq 0$. We consider waveforms $T_{k}(\tau)$ of type (35) such that $T_{k}(\tau) \geq 0$ and $T_{k}(\tau)=0$ for some $\tau_{0}$. From assumption that nonnegative waveform $T_{k}(\tau)$ of type (35) has at least one zero, it follows that it can be expressed in form (38).

Let us also assume that $\tau_{0}$ is position of nondegenerate critical point. Therefore $T_{k}\left(\tau_{0}\right)=0$ implies $T_{k}^{\prime}\left(\tau_{0}\right)=0$ and $T_{k}^{\prime \prime}\left(\tau_{0}\right)>0$. According to (55), second derivative of $T_{k}(\tau)$ at $\tau_{0}$ can be expressed as $T_{k}^{\prime \prime}\left(\tau_{0}\right)=1-\lambda_{k}\left(k^{2}-1\right) \cos \xi$. Since $T_{k}^{\prime \prime}\left(\tau_{0}\right)>0$ it follows immediately that

$$
1-\lambda_{k}\left(k^{2}-1\right) \cos \xi>0
$$

Let us further assume that $T_{k}(\tau)$ has exactly one zero. The problem of finding maximum absolute value of $a_{1}$ is connected to the problem of finding maximum of the minimum function (see Section 2.1). If waveforms possess unique global minimum at nondegenerate critical point then corresponding minimum function is a smooth function of parameters [13]. Consequently, assumption that $T_{k}(\tau)$ has exactly one zero at nondegenerate critical point leads to the conclusion that coefficient $a_{1}$ is differentiable function of $\tau_{0}$. First derivative of $a_{1}$ (see (43)) with respect to $\tau_{0}$, taking into account that $\partial \xi / \partial \tau_{0}=k$ (see (50)), can be expressed in the following factorized form:

$$
\frac{\partial a_{1}}{\partial \tau_{0}}=\sin \tau_{0}\left[1-\lambda_{k}\left(k^{2}-1\right) \cos \xi\right]
$$


From (138) and (139), it is clear that $\partial a_{1} / \partial \tau_{0}=0$ if and only if $\sin \tau_{0}=0$. According to Remark 12, assumption that $T_{k}(\tau)$ has exactly one zero implies $\lambda_{k}<1$. From (51), (48), and $\lambda_{k}<1$ it follows that $a_{1} \cos \tau_{0}+b_{1} \sin \tau_{0}<0$, which, together with $\sin \tau_{0}=0$, implies that $a_{1} \cos \tau_{0}<0$. Assumption $a_{1} \leq 0$, together with relations $a_{1} \cos \tau_{0}<0$ and $\sin \tau_{0}=0$, further implies $a_{1} \neq 0$ and

$$
\tau_{0}=0
$$

Insertion of $\tau_{0}=0$ into (38) leads to

$$
\begin{aligned}
& T_{k}(\tau) \\
& =[1-\cos \tau] \\
& \quad\left[1-(k-1) \lambda_{k} \cos \xi-2 \lambda_{k} \sum_{n=1}^{k-1}(k-n) \cos (n \tau+\xi)\right] .
\end{aligned}
$$

Substitution of $\tau_{0}=0$ into (45) and (46) yields $a_{k}=\lambda_{k} \cos \xi$ and $b_{k}=-\lambda_{k} \sin \xi$, respectively. Replacing $\lambda_{k} \cos \xi$ with $a_{k}$ and $\lambda_{k} \cos (n \tau+\xi)$ with $\left(a_{k} \cos n \tau+b_{k} \sin n \tau\right)$ in (141) immediately leads to (121).

Furthermore, $a_{k}=\lambda_{k} \cos \xi, b_{k}=-\lambda_{k} \sin \xi$, and (118) imply that

$$
\delta=-\xi
$$

According to (38)-(40) and (142), it follows that (141) is nonnegative if and only if

$$
\lambda_{k}\left[(k-1) \cos \delta+\frac{k \sin (\delta-\delta / k)}{\sin (\delta / k)}\right]<1 .
$$

Notice that $a_{k}=\lambda_{k} \cos \delta$ implies that the following relation holds:

$$
\begin{gathered}
\lambda_{k}\left[(k-1) \cos \delta+\frac{k \sin (\delta-\delta / k)}{\sin (\delta / k)}\right] \\
=-a_{k}+k \lambda_{k} \frac{\sin \delta}{\sin (\delta / k)} \cos \left(\frac{\delta}{k}\right) .
\end{gathered}
$$

Finally, substitution of (144) into (143) leads to $k \lambda_{k}[\sin \delta /$ $\sin (\delta / k)] \cos (\delta / k)<1+a_{k}$, which proves that (121) holds when $k \lambda_{k}[\sin \delta / \sin (\delta / k)] \cos (\delta / k)<1+a_{k}$.

Apart from nonnegative waveforms with exactly one zero at nondegenerate critical point, in what follows we will also consider other types of nonnegative waveforms with at least one zero. According to Proposition 9 and Remark 11, these waveforms can be described by (66)-(68) providing that $0 \leq$ $|\xi| \leq \pi$.

According to (35), $T_{k}(0) \geq 0$ implies $1+a_{1}+a_{k} \geq 0$. Consequently, $a_{1} \leq 0$ implies that $\left|a_{1}\right| \leq 1+a_{k}$. On the other hand, according to (123), $\left|a_{1}\right|=1+a_{k}$ holds for waveforms of type (121). The converse is also true; $a_{1} \leq 0$ and $\left|a_{1}\right|=$ $1+a_{k}$ imply $a_{1}=-1-a_{k}$, which further from (35) implies $T_{k}(0)=0$. Therefore, in what follows it is enough to consider only nonnegative waveforms which can be described by (66)(68) and $0 \leq|\xi| \leq \pi$, with coefficients $a_{k}$ and $b_{k}$ satisfying $k \lambda_{k}[\sin \delta / \sin (\delta / k)] \cos (\delta / k) \geq 1+a_{k}$.
For prescribed coefficients $a_{k}$ and $b_{k}$, the amplitude $\lambda_{k}=$ $\sqrt{a_{k}^{2}+b_{k}^{2}}$ of $k$ th harmonic is also prescribed. According to Remark 15 (see also Remark 16), $\lambda_{k}$ is monotonically decreasing function of $x=\cos (\xi / k)$. The value of $x$ can be obtained by solving (90) subject to the constraint $\cos (\pi / k) \leq$ $x \leq 1$. Then $\lambda_{1}$ can be determined from (88). From (106) it immediately follows that maximal absolute value of $a_{1} \leq 0$ corresponds to $q=0$, which from (104) and (120) further implies that

$$
\delta=k \tau_{0}-\xi
$$

Furthermore $q=0$, according to (107), implies that waveform zeros are

$$
\tau_{0}=\frac{(\delta+\xi)}{k}, \quad \tau_{0}^{\prime}=\tau_{0}-\frac{2 \xi}{k}=\frac{(\delta-\xi)}{k} .
$$

Substitution of $\tau_{0}=(\delta+\xi) / k$ into (66) yields (122), which proves that (122) holds when $k \lambda_{k}[\sin \delta / \sin (\delta / k)] \cos (\delta / k) \geq$ $1+a_{k}$.

In what follows we prove that (121) also holds when $k \lambda_{k}[\sin \delta / \sin (\delta / k)] \cos (\delta / k)=1+a_{k}$. Substitution of $a_{k}=$ $\lambda_{k} \cos \delta$ into $k \lambda_{k}[\sin \delta / \sin (\delta / k)] \cos (\delta / k)=1+a_{k}$ leads to

$$
\lambda_{k}\left[(k-1) \cos \delta+\frac{k \sin (\delta-\delta / k)}{\sin (\delta / k)}\right]=1 .
$$

As we mentioned earlier, relation (142) holds for all waveforms of type (121). Substituting (142) into (147) we obtain

$$
\lambda_{k}\left[(k-1) \cos \xi+k \frac{\sin (\xi-\xi / k)}{\sin (\xi / k)}\right]=1 .
$$

This expression can be rearranged as

$$
\lambda_{k} \frac{k \sin ((k-1) \xi / k)}{\sin \xi / k}=1-(k-1) \lambda_{k} \cos \xi
$$

On the other hand, for waveforms of type (122), according to (68), relations (148) and (149) also hold. Substitution of $\tau_{0}=$ $(\delta+\xi) / k($ see $(145))$ and (67) into (122) leads to

$$
\begin{aligned}
& T_{k}(\tau) \\
& =\lambda_{k}\left[1-\cos \left(\tau-\tau_{0}\right)\right] \\
& \quad\left[\frac{k \sin ((k-1) \xi / k)}{\sin \xi / k}-2 \sum_{n=1}^{k-1}(k-n) \cos \left(n\left(\tau-\tau_{0}\right)+\xi\right)\right] .
\end{aligned}
$$

Furthermore, substitution of (142) into (145) implies that $\tau_{0}=0$. Finally, substitution of $\tau_{0}=0$ and (149) into (150) leads to (141). Therefore (141) holds when $k \lambda_{k}[\sin \delta /$ $\sin (\delta / k)] \cos (\delta / k)=1+a_{k}$, which in turn shows that (121) holds when $k \lambda_{k}[\sin \delta / \sin (\delta / k)] \cos (\delta / k)=1+a_{k}$. This completes the proof. 
5.2. Nonnegative Waveforms with Maximal Absolute Value of Coefficient $a_{1}$ for $k=3$. Nonnegative waveform of type (35) for $k=3$ is widely used in PA design (e.g., see [10]). In this subsection we illustrate results of Section 5.1 for this particular case. The case $k=2$ is presented in detail in [12].

Coefficients of fundamental harmonic of nonnegative waveform of type (35) with $k=3$ and maximal absolute value of coefficient $a_{1} \leq 0$ for prescribed coefficients $a_{3}$ and $b_{3}\left(\lambda_{3}=\sqrt{a_{3}^{2}+b_{3}^{2}}\right)$, according to (123), (124), (134), (94), and (120), are equal to

$$
a_{1}=-1-a_{3}, \quad b_{1}=-3 b_{3},
$$

if $\lambda_{3}^{2} \leq\left[\left(1-2 a_{3}\right) / 3\right]^{3}$,

$$
a_{1}=-\lambda_{1} \cos \left(\frac{\delta}{3}\right), \quad b_{1}=-\lambda_{1} \sin \left(\frac{\delta}{3}\right),
$$

where $\lambda_{1}=3\left(\sqrt[3]{\lambda_{3}}-\lambda_{3}\right)$ and $\delta=\operatorname{atan} 2\left(b_{3}, a_{3}\right)$, if $[(1-$ $\left.\left.2 a_{3}\right) / 3\right]^{3} \leq \lambda_{3}^{2} \leq 1$. The line $\lambda_{3}^{2}=\left[\left(1-2 a_{3}\right) / 3\right]^{3}$ (see case $k=3$ in Figure 11) separates points representing waveforms with coefficients satisfying (151) from points representing waveforms with coefficients satisfying (152). Waveforms described by (151) for $\lambda_{3}^{2}<\left[\left(1-2 a_{3}\right) / 3\right]^{3}$ have exactly one zero at $\tau_{0}=0$. Waveforms described by (151) and (152) for $\lambda_{3}^{2}=[(1-$ $\left.\left.2 a_{3}\right) / 3\right]^{3}$ also have zero at $\tau_{0}=0$. These waveforms as a rule have exactly two zeros. However there are two exceptions: one related to the maximally flat nonnegative waveform with coefficients $a_{1}=-9 / 8, a_{3}=1 / 8$, and $b_{1}=b_{3}=0$, which has only one zero, and the other related to the waveform with coefficients $a_{1}=0, a_{3}=-1$, and $b_{1}=b_{3}=0$, which has three zeros. Waveforms described by (152) for $\left[\left(1-2 a_{3}\right) / 3\right]^{3}<\lambda_{3}^{2}<$ 1 have two zeros. Waveforms with $\lambda_{3}=1$ have only third harmonic (fundamental harmonic is zero).

Plot of contours of maximal absolute value of coefficient $a_{1}, a_{1} \leq 0$, for prescribed coefficients $a_{3}$ and $b_{3}$ is presented in Figure 12. According to Remark 25, the waveform with maximum absolute value of $a_{1} \leq 0$ is fully described with the following coefficients: $a_{1}=-2 / \sqrt{3}, a_{3}=\sqrt{3} / 9$ and $b_{1}=b_{3}=0$. This waveform has two zeros at $\pm \pi / 6$.

Two examples of nonnegative waveforms for $k=3$ and maximal absolute value of coefficient $a_{1}, a_{1} \leq 0$, with prescribed coefficients $a_{3}$ and $b_{3}$ are presented in Figure 13. One waveform corresponds to the case $\lambda_{3}^{2}<\left[\left(1-2 a_{3}\right) / 3\right]^{3}$ (solid line) and the other to the case $\lambda_{3}^{2}>\left[\left(1-2 a_{3}\right) / 3\right]^{3}$ (dashed line). The waveform represented by solid line has one zero and its coefficients are $a_{3}=-0.1, b_{3}=0.1, a_{1}=-0.9$, and $b_{1}=-0.3$. Dashed line corresponds to the waveform having two zeros with coefficients $a_{3}=-0.1, b_{3}=0.3, a_{1}=$ -0.8844 , and $b_{1}=-0.6460\left(\right.$ case $\left.\lambda_{3}^{2}>\left[\left(1-2 a_{3}\right) / 3\right]^{3}\right)$.

\section{Nonnegative Cosine Waveforms with at Least One Zero}

Nonnegative cosine waveforms have proved to be of importance for waveform modelling in PA design (e.g., see [10]). In this section we consider nonnegative cosine waveforms

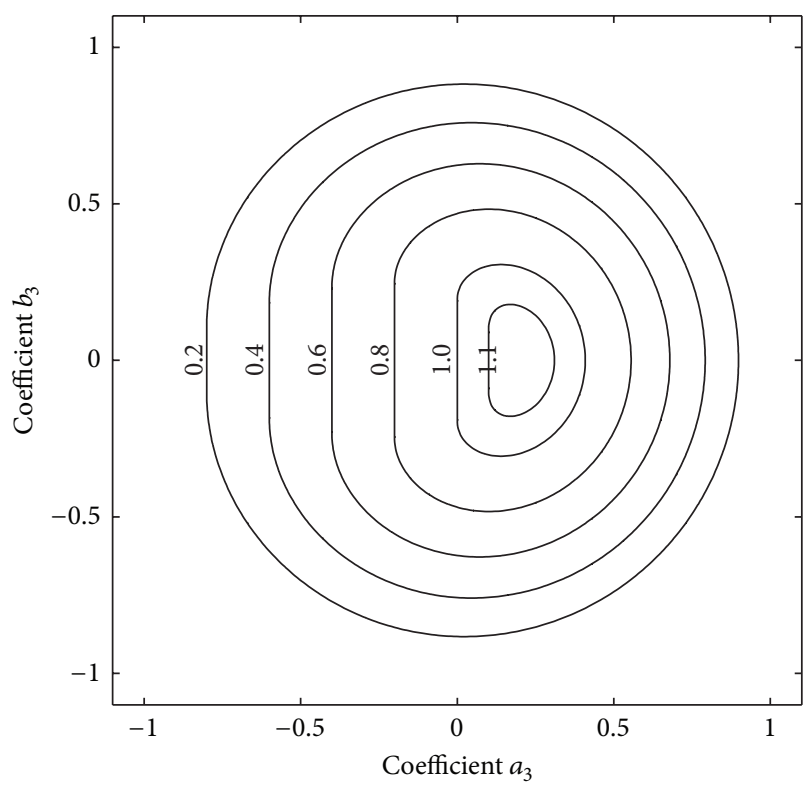

FIGURE 12: Contours of maximal absolute value of coefficient $a_{1}, a_{1} \leq$ 0 , as a function of $a_{3}$ and $b_{3}$.

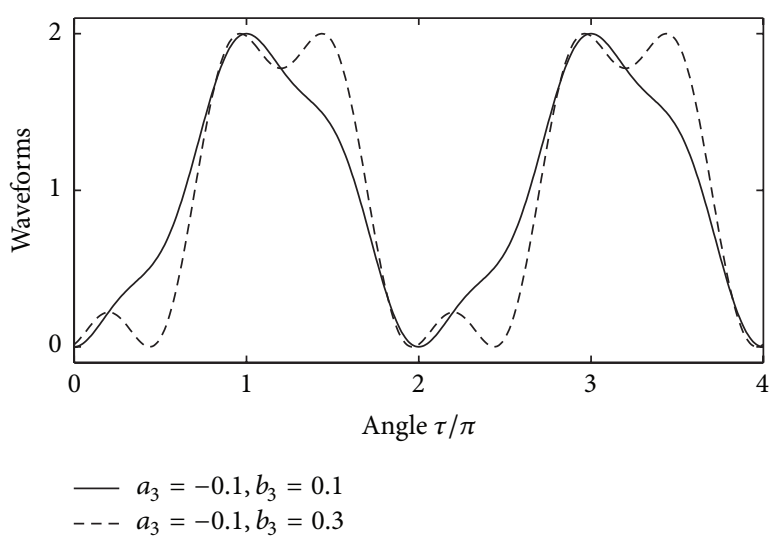

FIGURE 13: Nonnegative waveforms for $k=3$ and maximal absolute value of $a_{1}, a_{1} \leq 0$, with prescribed coefficients $a_{3}$ and $b_{3}$.

containing fundamental and $k$ th harmonic with at least one zero.

Cosine waveform with dc component, fundamental and $k$ th harmonic, can be obtained from (35) by setting $b_{1}=b_{k}=$ 0 ; that is,

$$
T_{k}(\tau)=1+a_{1} \cos \tau+a_{k} \cos k \tau .
$$

In Section 6.1 we provide general description of nonnegative cosine waveforms of type (153) with at least one zero. We show that nonnegative cosine waveforms with at least one zero coincide with nonnegative cosine waveforms with maximal absolute value of coefficient $a_{1}$ for prescribed coefficient $a_{k}$. In Section 6.2 we illustrate results of Section 6.1 for particular case $k=3$.

6.1. Nonnegative Cosine Waveforms with at Least One Zero for $k \geq 2$. Amplitudes of fundamental and $k$ th harmonic 
of cosine waveform of type (153) are $\lambda_{1}=\left|a_{1}\right|$ and $\lambda_{k}=$ $\left|a_{k}\right|$, respectively. According to (42), for nonnegative cosine waveforms of type (153) the following relation holds:

$$
-1 \leq a_{k} \leq 1
$$

This explains why $k$ th harmonic coefficient $a_{k}$ in Proposition 26 goes through interval $[-1,1]$.

Waveforms (153) with $a_{1} \geq 0$ can be obtained from waveforms with $a_{1} \leq 0$ by shifting by $\pi$, and therefore, without loss of generality, we can assume that $a_{1} \leq 0$.

Proposition 26. Each nonnegative cosine waveform of type (153) with $a_{1} \leq 0$ and at least one zero can be represented as

$$
T_{k}(\tau)=[1-\cos \tau]\left[1-(k-1) a_{k}-2 a_{k} \sum_{n=1}^{k-1}(k-n) \cos n \tau\right],
$$

$$
\begin{aligned}
& \text { if }-1 \leq a_{k} \leq 1 /\left(k^{2}-1\right) \text {, or } \\
& \begin{aligned}
T_{k}(\tau)= & a_{k}\left[1-\cos \left(\tau-\tau_{0}\right)\right]\left[1-\cos \left(\tau+\tau_{0}\right)\right] \\
& \cdot\left[c_{0}+2 \sum_{n=1}^{k-2} c_{n} \cos n \tau\right],
\end{aligned}
\end{aligned}
$$

where

$$
\begin{gathered}
c_{n}=\frac{\sin \left((k-n) \tau_{0}\right) \cos \tau_{0}-(k-n) \cos \left((k-n) \tau_{0}\right) \sin \tau_{0}}{\sin ^{3} \tau_{0}} \\
a_{k}=\frac{\sin \tau_{0}}{k \sin \left(k \tau_{0}\right) \cos \tau_{0}-\cos \left(k \tau_{0}\right) \sin \tau_{0}} \\
\left|\tau_{0}\right| \leq \frac{\pi}{k}
\end{gathered}
$$

if $1 /\left(k^{2}-1\right) \leq a_{k} \leq 1$.

Remark 27. Identity $\left[1-\cos \left(\tau-\tau_{0}\right)\right]\left[1-\cos \left(\tau+\tau_{0}\right)\right]=\left[\cos \tau_{0}-\right.$ $\cos \tau]^{2}$ implies that (156) can be rewritten as

$$
T_{k}(\tau)=a_{k}\left[\cos \tau_{0}-\cos \tau\right]^{2}\left[c_{0}+2 \sum_{n=1}^{k-2} c_{n} \cos n \tau\right] .
$$

Furthermore, substitution of (157) into (160) leads to

$$
\begin{aligned}
T_{k}(\tau)= & a_{k}\left[\cos \tau_{0}-\cos \tau\right] \\
& \cdot\left[\frac{(k-1) \sin k \tau_{0}}{\sin \tau_{0}}-2 \sum_{n=1}^{k-1} \frac{\sin \left((k-n) \tau_{0}\right)}{\sin \tau_{0}} \cos n \tau\right] .
\end{aligned}
$$

Remark 28. All nonnegative cosine waveforms of type (153) with at least one zero and $a_{1} \leq 0$, except one of them, can be represented either in form (155) or form (156). This exception is maximally flat cosine waveform with $a_{1}<0$ which can be obtained from (155) for $a_{k}=1 /\left(k^{2}-1\right)$ or from (156) for $\tau_{0}=$ 0 . Maximally flat cosine waveform with $a_{1}<0$ can also be obtained from (70) by setting $\tau_{0}=0$. Furthermore, setting $\tau_{0}=0$ in (71) leads to maximally flat cosine waveforms for $k \leq 4$ and $a_{1}<0$.
Remark 29. Nonnegative cosine waveform of type (155) with $a_{1}<0$ and $-1<a_{k} \leq 1 /\left(k^{2}-1\right)$ has exactly one zero at $\tau=0$. Nonnegative cosine waveform described by (156) with $a_{1}<0$ and $1 /\left(k^{2}-1\right)<a_{k}<1$ has two zeros at $\pm \tau_{0}$, where $0<\left|\tau_{0}\right|<\pi / k$. For $a_{k}=\mp 1$, nonnegative cosine waveform of type (153) reduces to $T_{k}(\tau)=1 \mp \cos k \tau$ (clearly, these two waveforms both have $k$ zeros).

Remark 30. Transformation of (155) into an additive form leads to the following relation:

$$
a_{1}=-1-a_{k}
$$

where $-1 \leq a_{k} \leq 1 /\left(k^{2}-1\right)$. Similarly, transformation of (156) leads to the following relation:

$$
a_{1}=-a_{k} \frac{k \sin k \tau_{0}}{\sin \tau_{0}}
$$

where $a_{k}$ is given by (158), $1 /\left(k^{2}-1\right) \leq a_{k} \leq 1$, and $\left|\tau_{0}\right| \leq \pi / k$. Notice that coefficients of maximally flat cosine waveform, namely, $a_{k}=1 /\left(k^{2}-1\right)$ and $a_{1}=-k^{2} /\left(k^{2}-1\right)$, satisfy relation (162). They also satisfy relation (163) for $\tau_{0}=0$.

Remark 31. Nonnegative cosine waveforms of type (153) with at least one zero coincide with nonnegative cosine waveforms with maximal absolute value of coefficient $a_{1}$ for prescribed coefficient $a_{k}$.

In proving that Remark 31 holds, notice that expression (155) can be obtained from (121) by setting $b_{k}=0$. Furthermore, if $a_{k} \geq 0$, then $\lambda_{k}=a_{k}$, which together with $b_{k}=0$ and (118) implies $\delta=0$. In this case $k \lambda_{k}[\sin \delta / \sin (\delta / k)] \cos (\delta / k) \leq 1+a_{k}$ becomes $k^{2} a_{k} \leq 1+$ $a_{k}$. On the other hand, if $a_{k}<0$, then $\lambda_{k}=-a_{k}$, which together with $b_{k}=0$ and (118) implies $|\delta|=\pi$. In this case $k \lambda_{k}[\sin \delta / \sin (\delta / k)] \cos (\delta / k) \leq 1+a_{k}$ becomes $0 \leq 1+a_{k}$. Therefore, every nonnegative cosine waveform of type (155) has maximal absolute value of coefficient $a_{1}$ for prescribed coefficient $a_{k}$, when $-1 \leq a_{k} \leq 1 /\left(k^{2}-1\right)$.

Let us now show that expression (156) can be obtained from (122) by setting $b_{k}=0$ and $a_{k}>0$. For waveforms of type (122), according to (118), $b_{k}=0$ and $a_{k}>0$ imply $\delta=0$ and $\lambda_{k}=a_{k}$. Substitution of $\lambda_{k}=a_{k}$ and $\delta=0$ into $k \lambda_{k}[\sin \delta / \sin (\delta / k)] \cos (\delta / k) \geq 1+a_{k}$ leads to $a_{k} \geq 1 /\left(k^{2}-1\right)$. Furthermore, substitution of $\delta=0$ into (145) yields $\tau_{0}=\xi / k$. Insertion of $\lambda_{k}=a_{k}, \delta=0$, and $\tau_{0}=\xi / k$ into (122) leads to (156). Therefore, every nonnegative cosine waveform of type (156) has maximal absolute value of coefficient $a_{1}$ for prescribed coefficient $a_{k}$, when $1 /\left(k^{2}-1\right) \leq a_{k} \leq 1$.

Proof of Proposition 26. Let us start with nonnegative cosine waveform of type (153) with $\lambda_{k}=\left|a_{k}\right|=1$. According to Remark 7, $\lambda_{k}=\left|a_{k}\right|=1$ implies that $\lambda_{1}=\left|a_{1}\right|=0$. Substitution of $a_{k}=-1$ into (155) and using (A.2) (see Appendices) lead to $T_{k}(\tau)=1-\cos k \tau$. Consequently, (155) holds for $a_{k}=-1$. On the other hand, substitution of $a_{k}=1$ into (158) yields $\left|\tau_{0}\right|=\pi / k$. Furthermore, substitution of $a_{k}=1$ and $\tau_{0}=\pi / k$ (or $\tau_{0}=-\pi / k$ ) into (156), along with performing all multiplications and using (A.2), leads to 
$T_{k}(\tau)=1+\cos k \tau$. Consequently, (156)-(158) hold for $a_{k}=1$ and $\left|\tau_{0}\right|=\pi / k$.

It is easy to see that $\lambda_{k}=\left|a_{k}\right|<1$ and $T_{k}\left(\tau_{0}\right)=0$ for some $\tau_{0}$ imply $\lambda_{1}=\left|a_{1}\right| \neq 0$. Therefore in what follows we assume that $\left|a_{k}\right| \neq 1$ and $a_{1}<0$.

Cosine waveforms are even functions of $\tau$. Therefore, if nonnegative cosine waveform has exactly one zero it has to be either at 0 or at $\pi$. On the other hand, if nonnegative cosine waveform with $a_{1} \neq 0$ has exactly two zeros then these zeros are placed at $\pm \tau_{0}$, such that $\tau_{0}$ is neither 0 nor $\pi$.

In order to prove that (155) holds for $-1<a_{k} \leq 1 /\left(1-k^{2}\right)$, let us start by referring to the description (38) of nonnegative waveforms with at least one zero. As we mentioned earlier, for nonnegative cosine waveform with exactly one zero (denoted by $\tau_{0}$ ) it is either $\tau_{0}=0$ or $\tau_{0}=\pi$. Therefore in both cases $\sin \tau_{0}=0$. Substitution of $\sin \tau_{0}=0$ into (43), together with $a_{1} \neq 0$ and $\lambda_{k}=\left|a_{k}\right|<1$, leads to

$$
\tau_{0}=0 .
$$

Clearly $\tau_{0}=0, b_{1}=0$, and $b_{k}=0$, according to (44) and (46), imply $\lambda_{k} \sin \xi=0$. Since $\lambda_{k}=\left|a_{k}\right|$ it follows that $\left|a_{k}\right| \sin \xi=0$ also holds, which further implies $\lambda_{k}=a_{k}=0$ or $\sin \xi=$ 0 . In the case when $\lambda_{k}=a_{k}=0$, from (164) and (43) we obtain $a_{1}=-1$, which further implies that $T_{k}(\tau)=1-\cos \tau$. Consequently (155) holds for $a_{k}=0$. In the case when $\sin \xi=$ 0 , from (164) and (45) we obtain $a_{k}=\lambda_{k}$ if $\xi=0$, or $a_{k}=-\lambda_{k}$ if $\xi=\pi$. Relations $a_{k}=\lambda_{k}$ and $\xi=0$, according to (40), imply that $0 \leq a_{k} \leq 1 /\left(1-k^{2}\right)$. Substitution of $\xi=0, \lambda_{k}=a_{k}$, and (164) into (38) leads to (155), which proves that (155) holds for $0 \leq a_{k} \leq 1 /\left(1-k^{2}\right)$. On the other hand, relations $a_{k}=$ $-\lambda_{k}$ and $\xi=\pi$, according to (40), imply that $-1<a_{k} \leq 0$. Substitution of $\xi=\pi, \lambda_{k}=-a_{k}$, and (164) into (38) also leads to (155), which proves that (155) also holds for $-1<a_{k} \leq 0$. Consequently (155) holds for $-1<a_{k} \leq 1 /\left(1-k^{2}\right)$.

In what follows we first prove that (156)-(157) hold for $1 /\left(1-k^{2}\right)<a_{k}<1$. For this purpose let us start with nonnegative waveforms with two zeros described by (66). As we mentioned before, nonnegative cosine waveforms with two zeros have zeros at $\tau_{0}$ and $-\tau_{0}$, such that $\tau_{0} \neq 0$ and $\tau_{0} \neq \pi$. Relations $a_{1}<0$ and $b_{1}=0$, according to (84), imply $\cos \left(\tau_{0}-\xi / k\right)=1$ and therefore

$$
\frac{\xi}{k}=\tau_{0} .
$$

From $\xi / k=\tau_{0}$ and $0<|\xi|<\pi$ it follows that $0<\left|\tau_{0}\right|<$ $\pi / k$. Insertion of $\xi / k=\tau_{0}$ into (45) yields $a_{k}=\lambda_{k}$. Relations $a_{k}=\lambda_{k}$ and (82) imply that $1 /\left(1-k^{2}\right)<a_{k}<1$. Substitution of $\lambda_{k}=a_{k}$ and $\xi / k=\tau_{0}$ into (66)-(68) leads to (156)-(158), which proves that (156)-(158) hold for $1 /\left(1-k^{2}\right)<a_{k}<1$ and $0<\left|\tau_{0}\right|<\pi / k$.

Finally, substitution of $a_{k}=1 /\left(1-k^{2}\right)$ and $\tau_{0}=0$ into (161) leads to

$$
T_{k}(\tau)=\frac{[1-\cos \tau]}{\left(1-k^{2}\right)}\left[k(k-1)-2 \sum_{n=1}^{k-1}(k-n) \cos n \tau\right] .
$$

Waveform (166) coincides with waveform (155) for $a_{k}=$ $1 /\left(1-k^{2}\right)$, which in turn proves that (156) holds for $a_{k}=$ $1 /\left(1-k^{2}\right)$ and $\tau_{0}=0$. This completes the proof.

6.2. Nonnegative Cosine Waveforms with at Least One Zero for $k=3$. In this subsection we consider nonnegative cosine waveforms with at least one zero for $k=3$ (for case $k=2$ see [12]).

Cosine waveform with fundamental and third harmonic reads

$$
T_{3}(\tau)=1+a_{1} \cos \tau+a_{3} \cos 3 \tau .
$$

For $a_{1} \leq 0$ and $-1 \leq a_{3} \leq 1 / 8$, according to (155), nonnegative cosine waveform of type (167) with at least one zero can be expressed as

$$
T_{3}(\tau)=(1-\cos \tau)\left[1-2 a_{3}(1+2 \cos \tau+\cos 2 \tau)\right] .
$$

From $T_{3}(\tau+\pi)=2-T_{3}(\tau)$, it immediately follows that, for $a_{1} \geq 0$ and $-1 / 8 \leq a_{3} \leq 1, T_{3}(\tau)$ can be expressed as

$$
T_{3}(\tau)=(1+\cos \tau)\left[1+2 a_{3}(1-2 \cos \tau+\cos 2 \tau)\right] .
$$

For $a_{1} \leq 0$ and $1 / 8 \leq a_{3} \leq 1$, from (158) it follows that $a_{3}=$ $\left[8 \cos ^{3} \tau_{0}\right]^{-1}$. This relation, along with (160) and (157), further implies that $T_{3}(\tau)$ can be expressed as

$$
T_{3}(\tau)=\frac{\left[\cos \tau_{0}-\cos \tau\right]^{2}\left[2 \cos \tau_{0}+\cos \tau\right]}{2 \cos ^{3} \tau_{0}},
$$

providing that $\left|\tau_{0}\right| \leq \pi / 3$. From $T_{3}(\tau+\pi)=2-T_{3}(\tau)$, it follows that (170) also holds for $a_{1} \geq 0$ and $-1 \leq a_{3} \leq-1 / 8$, providing that $\tau_{0} \in[2 \pi / 3,4 \pi / 3]$.

Maximally flat nonnegative cosine waveform of type (167) with $a_{1}<0$ (minimum at $\tau_{0}=0$ ) reads $T_{3}(\tau)=[1-$ $\cos \tau]^{2}[1+(1 / 2) \cos \tau]$. Dually, maximally flat nonnegative cosine waveform with $a_{1}>0$ (minimum at $\tau_{0}=\pi$ ) reads $T_{3}(\tau)=[1+\cos \tau]^{2}[1-(1 / 2) \cos \tau]$.

In what follows we provide relations between coefficients $a_{1}$ and $a_{3}$ of nonnegative cosine waveforms of type (167) with at least one zero.

For $a_{1} \leq 0$, conversion of (168) into an additive form immediately leads to the following relation:

$$
a_{1}=-1-a_{3} \text { for }-1 \leq a_{3} \leq \frac{1}{8} .
$$

Conversion of (170) into an additive form leads to $a_{1}=$ $-3 a_{3}\left(2 \cos 2 \tau_{0}+1\right)$, which can be also expressed as $a_{1}=$ $-3 a_{3}\left(4 \cos ^{2} \tau_{0}-1\right)$. For $a_{1} \leq 0$, relations $\left|\tau_{0}\right| \leq \pi / 3, a_{1}=$ $-3 a_{3}\left(4 \cos ^{2} \tau_{0}-1\right)$, and $a_{3}=\left[8 \cos ^{3} \tau_{0}\right]^{-1}$ lead to

$$
a_{1}=-3\left[\sqrt[3]{a_{3}}-a_{3}\right] \quad \text { for } \frac{1}{8} \leq a_{3} \leq 1 .
$$

Similarly for $a_{1} \geq 0$, conversion of (169) into an additive form leads to the following relation:

$$
a_{1}=1-a_{3} \text { for }-\frac{1}{8} \leq a_{3} \leq 1 .
$$




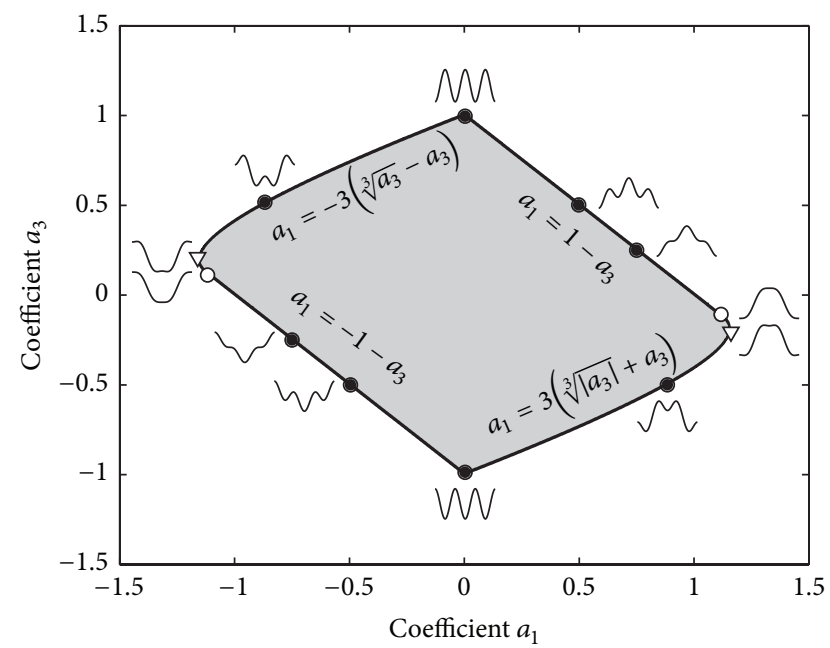

Figure 14: Parameter space of cosine waveforms for $k=3$.

For waveform of type (170) with $a_{1} \geq 0$, relations $\tau_{0} \in[2 \pi / 3$, $4 \pi / 3], a_{1}=-3 a_{3}\left(4 \cos ^{2} \tau_{0}-1\right)$, and $a_{3}=\left[8 \cos ^{3} \tau_{0}\right]^{-1}$ lead to

$$
a_{1}=3\left[\sqrt[3]{\left|a_{3}\right|}+a_{3}\right] \quad \text { for }-1 \leq a_{3} \leq-\frac{1}{8} .
$$

Every cosine waveform of type (167) corresponds to a pair of real numbers $\left(a_{1}, a_{3}\right)$ and vice versa. Points $\left(a_{1}, a_{3}\right)$ in grey area in Figure 14 correspond to nonnegative cosine waveforms for $k=3$. The points at the boundary of grey area correspond to nonnegative cosine waveforms with at least one zero. A number of shapes of nonnegative cosine waveforms with $k=3$ and at least one zero, plotted on interval $[-\pi, \pi]$, are also presented in Figure 14. The boundary of grey area in Figure 14 consists of four line segments described by relations (171)-(174). The common point of line segments (172) and (173) is cusp point with coordinates $a_{1}=0$ and $a_{3}=1$. Another cusp point, with coordinates $a_{1}=0$ and $a_{3}=-1$, is the common point of line segments (171) and (174). The common point of line segments (171)-(172) has coordinates $(-9 / 8,1 / 8)$ and common point of line segments (173)-(174) has coordinates $(9 / 8,-1 / 8)$. These points are represented by white circle dots and they correspond to maximally flat cosine waveforms (e.g., see [21]). White triangle dots with coordinates $(2 / \sqrt{3},-\sqrt{3} / 9)$ and $(-2 / \sqrt{3}, \sqrt{3} / 9)$ refer to the nonnegative cosine waveforms with maximum value of amplitude of fundamental harmonic.

\section{Four Case Studies of Usage of Nonnegative Waveforms in PA Efficiency Analysis}

In this section we provide four case studies of usage of description of nonnegative waveforms with fundamental and $k$ th harmonic in PA efficiency analysis. In first two case studies, to be presented in Section 7.1, voltage is nonnegative waveform with fundamental and second harmonic with at least one zero. In remaining two case studies, to be considered in Section 7.2, voltage waveform contains fundamental and third harmonic.

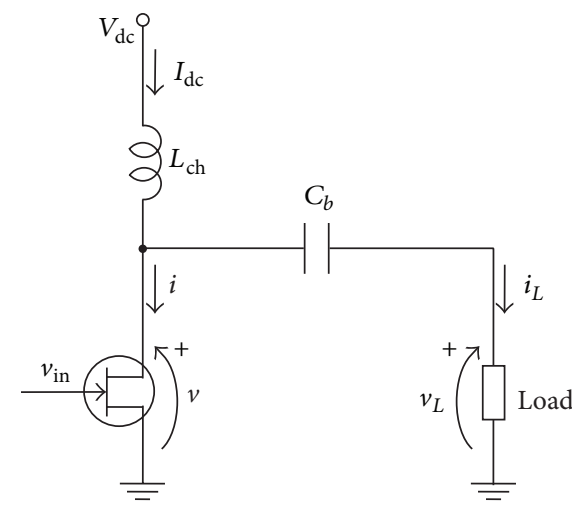

FIGURE 15: Generic PA circuit diagram.

Let us consider generic PA circuit diagram, as shown in Figure 15. We assume here that voltage and current waveforms at the transistor output are

$$
\begin{gathered}
v(\theta)=1+a_{1 v} \cos \theta+b_{1 v} \sin \theta+a_{k v} \cos k \theta+b_{k v} \sin k \theta, \\
i(\theta)=1+a_{1 i} \cos \theta+\sum_{n=2}^{\infty} a_{n i} \cos n \theta
\end{gathered}
$$

where $\theta$ stands for $\omega t$. Both waveforms are normalized in the sense that $\mathrm{dc}$ components of voltage and current are $V_{\mathrm{dc}}=$ 1 and $I_{\mathrm{dc}}=1$, respectively. Under assumption that blocking capacitor $C_{b}$ behaves as short-circuit at the fundamental and higher harmonics, current and voltage waveforms at the load are

$$
\begin{gathered}
v_{L}(\theta)=a_{1 v} \cos \theta+b_{1 v} \sin \theta+a_{k v} \cos k \theta+b_{k v} \sin k \theta \\
i_{L}(\theta)=-a_{1 i} \cos \theta-\sum_{n=2}^{\infty} a_{n i} \cos n \theta
\end{gathered}
$$

In terms of coefficients of voltage and current waveforms, the load impedance at fundamental harmonic is $\underline{z}_{1}=-\left(a_{1 v}-\right.$ $\left.j b_{1 v}\right) / a_{1 i}$, whereas load impedance at $k$ th harmonic is $\underline{z}_{k}=$ $-\left(a_{k v}-j b_{k v}\right) / a_{k i}$. All other harmonics are short-circuited $\left(\underline{z}_{n}=\right.$ 0 for $n \neq 1$ and $n \neq k$ ). Time average output power of PA (e.g., see [10]) with waveform pair (175) at fundamental frequency can be expressed as

$$
P_{1}=-\frac{a_{1 i} a_{1 v}}{2}
$$

For normalized waveforms (175) with $V_{\mathrm{dc}}=1$ and $I_{\mathrm{dc}}=1$, dc power is $P_{\mathrm{dc}}=1$. Consequently, PA efficiency $\eta=P_{1} / P_{\mathrm{dc}}$ (e.g., see, $[10,26])$ is equal to

$$
\eta=-\frac{a_{1 i} a_{1 v}}{2}
$$

Thus, time average output power $P_{1}$ of PA with pair of normalized waveform (175) is equal to efficiency (178).

Power utilization factor (PUF) is defined [26] as "the ratio of power delivered in a given situation to the power 
delivered by the same device with the same supply voltage in Class A mode." Since the output power in class-A mode is $P_{1 \text {,class-A }}=\max [v(\theta)] \cdot \max [i(\theta)] / 8$ (e.g., see [9]), it follows that power utilization factor PUF $=P_{1} / P_{1 \text {,class-A }}$ for PA with pair of normalized waveforms (175) can be expressed as

$$
\mathrm{PUF}=\frac{8 \eta}{\max [v(\theta)] \cdot \max [i(\theta)]} .
$$

7.1. Nonnegative Waveforms for $k=2$ in PA Efficiency Analysis. In this subsection we provide two case studies of usage of description of nonnegative waveforms with fundamental and second harmonic $(k=2)$ in PA efficiency analysis. For more examples of usage of descriptions of nonnegative waveforms with fundamental and second harmonic in PA efficiency analysis see [12].

Case Study 7.1. In this case study we consider efficiency of PA for given second harmonic impedance, providing that voltage is nonnegative waveform with fundamental and second harmonic and current is "half-sine" waveform frequently used in efficiency analysis of classical PA operation (e.g., see [10]).

Standard model of current waveform for classical PA operation has the form (e.g., see $[10,26]$ )

$$
i_{D}(\theta)= \begin{cases}I_{D}\left[\cos \theta-\cos \left(\frac{\alpha}{2}\right)\right], & |\theta| \leq \frac{\alpha}{2} \\ 0, & \frac{\alpha}{2} \leq|\theta| \leq \pi,\end{cases}
$$

where $\alpha$ is conduction angle and $I_{D}>0$. Since $i_{D}(\theta)$ is even function, it immediately follows that its Fourier series contains only dc component and cosine terms:

$$
i_{D}(\theta)=I_{\mathrm{dc}}+\sum_{n=1}^{\infty} I_{n} \cos n \theta
$$

The dc component of the waveform (180) is

$$
I_{\mathrm{dc}}=\frac{I_{D} \alpha}{2 \pi}\left[\operatorname{sinc}\left(\frac{\alpha}{2}\right)-\cos \left(\frac{\alpha}{2}\right)\right],
$$

where $\operatorname{sinc} x=(\sin x) / x$. The coefficient of the fundamental harmonic component reads

$$
I_{1}=\frac{I_{D} \alpha}{2 \pi}(1-\operatorname{sinc} \alpha)
$$

and the coefficient of $n$th harmonic component can be written in the form

$$
I_{n}=\frac{I_{D}}{n \pi}\left[\frac{\sin ((n-1) \alpha / 2)}{(n-1)}-\frac{\sin ((n+1) \alpha / 2)}{(n+1)}\right], \quad n \geq 2 .
$$

For "half-sine" current waveform, conduction angle is equal to $\pi$ (class-B conduction angle). According to (182), this further implies that $I_{\mathrm{dc}}=I_{D} / \pi$. To obtain normalized form of waveform (180), we set $I_{\mathrm{dc}}=1$ which implies that $I_{D}=\pi$. Furthermore, substitution of $\alpha=\pi$ and $I_{D}=\pi$ in (180) leads to

$$
i(\theta)= \begin{cases}\pi \cos \theta, & |\theta|<\frac{\pi}{2}, \\ 0, & \frac{\pi}{2}<|\theta| \leq \pi .\end{cases}
$$

Similarly, substitution of $I_{D}=\pi$ and $\alpha=\pi$ into (183) and (184) leads to the coefficients of waveform (185). Coefficients of fundamental and second harmonic, respectively, are

$$
a_{1 i}=\frac{\pi}{2}, \quad a_{2 i}=\frac{2}{3} .
$$

On the other hand, voltage waveform of type (35) for $k=$ 2 reads

$$
v(\theta)=1+a_{1 v} \cos \theta+b_{1 v} \sin \theta+a_{2 v} \cos 2 \theta+b_{2 v} \sin 2 \theta .
$$

This waveform contains only fundamental and second harmonic, and therefore all harmonics of order higher than two are short-circuited $\left(\underline{z}_{n}=0\right.$ for $\left.n>2\right)$. For current voltage pair (185) and (187), load impedance at fundamental harmonic is $\underline{z}_{1}=-\left(a_{1 v}-j b_{1 v}\right) / a_{1 i}$, whereas load impedance at second harmonic is $\underline{z}_{2}=-\left(a_{2 v}-j b_{2 v}\right) / a_{2 i}$. According to our assumption, the load is passive and therefore $\operatorname{Re}\left\{\underline{z}_{1}\right\}>0$ and $\operatorname{Re}\left\{\underline{z}_{2}\right\} \geq 0$, which further imply $a_{1 i} a_{1 v}<0$ and $a_{2 i} a_{2 v} \leq 0$, respectively.

It is easy to see that problem of finding maximal efficiency of PA with current-voltage pair (185) and (187) for prescribed second harmonic impedance can be reduced to the problem of finding voltage waveform of type (187) with maximal coefficient $\left|a_{1 v}\right|$, for prescribed coefficients of second harmonic (see Section 5).

The following algorithm (analogous to Algorithm 22 presented in [12]) provides the procedure for calculation of maximal efficiency with current-voltage pair (185) and (187) for prescribed second harmonic impedance. The definition of function atan $2(y, x)$, which appears in the step (iii) of the following algorithm, is given by (105).

Algorithm 32. (i) Choose $\underline{z}_{2}=r_{2}+j x_{2}$ such that $\left|\underline{z}_{2}\right| \leq 1 /\left|a_{2 i}\right|$,

(ii) calculate $a_{2 v}-j b_{2 v}=-\underline{z}_{2} a_{2 i}$ and $\lambda_{2 v}=\sqrt{a_{2 v}^{2}+b_{2 v}^{2}}$,

(iii) if $2 \lambda_{2 v} \leq 1-a_{2 v}$ then calculate $a_{1 v}=-1-a_{2 v}$ and $b_{1 v}=-2 b_{2 v}$; else, calculate $\lambda_{1 v}=\sqrt{8 \lambda_{2 v}\left(1-\lambda_{2 v}\right)}, \theta_{0 v}-\xi_{v} / 2=$ $(1 / 2) \operatorname{atan} 2\left(b_{2 v}, a_{2 v}\right), a_{1 v}=-\lambda_{1 v} \cos \left(\theta_{0 v}-\xi_{v} / 2\right)$, and $b_{1 v}=$ $-\lambda_{1 v} \sin \left(\theta_{0 v}-\xi_{v} / 2\right)$,

(iv) calculate efficiency $\eta=-a_{1 i} a_{1 v} / 2$,

(v) calculate $\underline{z}_{1}=-\left(a_{1 v}-j b_{1 v}\right) / a_{1 i}$ and $\underline{z}_{2 n}=\underline{z}_{2} / \operatorname{Re}\left\{\underline{z}_{1}\right\}$.

In this case study, coefficients of fundamental and second harmonic of current waveform are given by (186). Maximal efficiency of PA associated with the waveform pair (185) and (187), as a function of normalized second harmonic impedance $\underline{z}_{2 n}=\underline{z}_{2} / \operatorname{Re}\left\{\underline{z}_{1}\right\}$, is presented in Figure 16(a). As can be seen from Figure 16(a), efficiency of 0.78 is achieved at the edge of Smith chart, where second harmonic impedance has small resistive part. Corresponding PUF calculated according to (179) is presented in Figure 16(b). Peak efficiency $\eta=\pi / 4=0.7854$ and peak value of PUF $=1$ are attained when second harmonic is short-circuited (which corresponds to ideal class-B operation $[10,26])$.

For example, for second harmonic impedance $\underline{z}_{2}=0.1-$ $j 0.5$ and current waveform (185), from Algorithm 32 it follows that $2 \lambda_{2 v} \leq 1-a_{2 v}$. Furthermore, according to step (iii) of above algorithm, maximal efficiency of PA is attained 


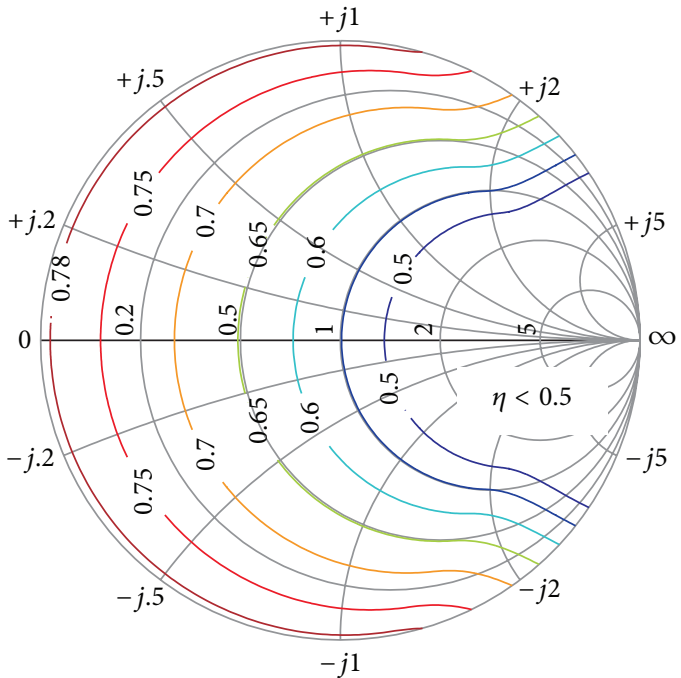

(a)

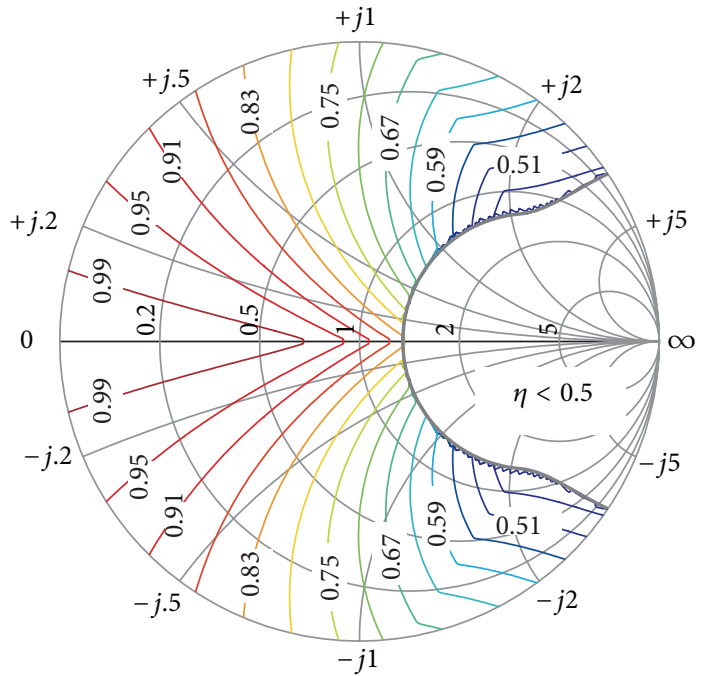

(b)

FIGURE 16: (a) Contours of maximal efficiency of PA and (b) contours of corresponding PUF, associated with the waveform pair (185) and (187), as functions of normalized second harmonic impedance $\underline{z}_{2 n}=\underline{z}_{2} / \operatorname{Re}\left\{\underline{z}_{1}\right\}$.

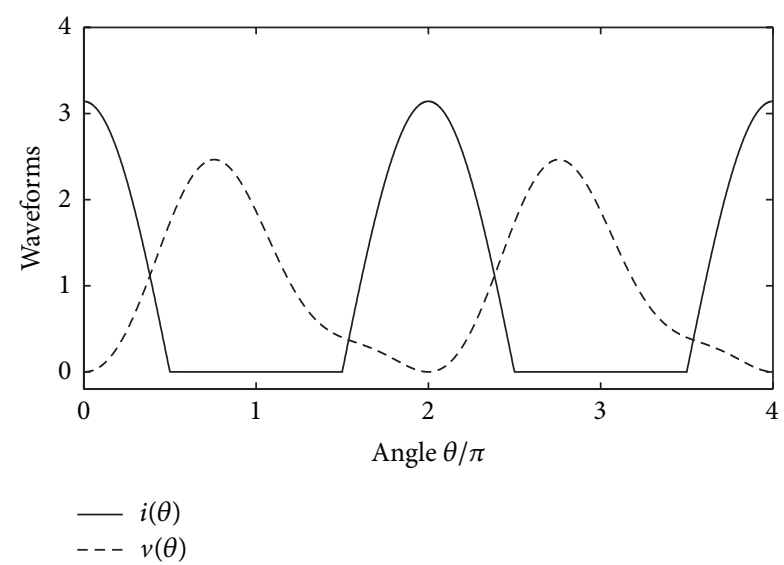

FIGURE 17: Waveform pair (185) and (187) that provides maximal efficiency for $\underline{z}_{2}=0.1-j 0.5$.

with voltage waveform of type (187) with coefficients $a_{2 v}=$ $-0.0667, b_{2 v}=-0.3333, a_{1 v}=-0.9333$, and $b_{1 v}=0.6667$ (see Figure 17). Corresponding efficiency, PUF, and normalized second harmonic impedance are $\eta=0.7330, \mathrm{PUF}=0.7572$, and $\underline{z}_{2 n}=0.1683-j 0.8415$, respectively.

On the other hand, for second harmonic impedance $\underline{z}_{2}=$ $0.1-j 0.8$ and current waveform (185), from Algorithm 32 it follows that $2 \lambda_{2 v}>1-a_{2 v}$. Then, according to step (iii) of above algorithm, maximal efficiency is attained with voltage waveform of type (187) with coefficients $a_{2 v}=-0.0667, b_{2 v}=$ $-0.5333, a_{1 v}=-0.9333$, and $b_{1 v}=1.0572$ (see Figure 18). Efficiency, PUF, and normalized second harmonic impedance are $\eta=0.7330$, PUF $=0.6332$, and $\underline{z}_{2 n}=0.1683-j 1.3465$, respectively.

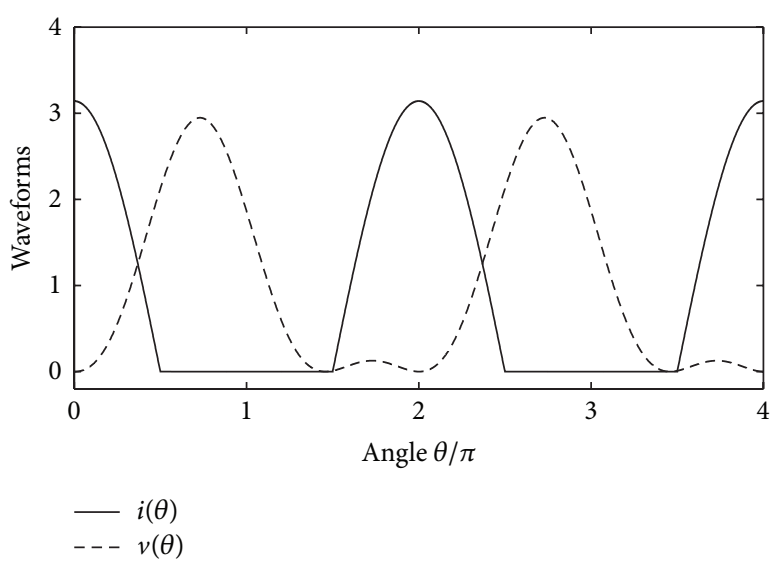

FIGURE 18: Waveform pair (185) and (187) that provides maximal efficiency for $\underline{z}_{2}=0.1-j 0.8$.

Case Study 7.2. As another case study, let us consider the efficiency of PA, providing that current waveform is nonnegative cosine waveform up to third harmonic with maximum value of amplitude of fundamental harmonic [22] (see also [8]):

$$
i(\theta)=1+\frac{1+\sqrt{5}}{2} \cos \theta+\frac{2 \sqrt{5}}{5} \cos 2 \theta+\frac{5-\sqrt{5}}{10} \cos 3 \theta,
$$

and voltage waveform is nonnegative waveform of type (187). Load impedances at fundamental, second, and third harmonic are $z_{1}=-\left(a_{1 v}-j b_{1 v}\right) / a_{1 i}, \underline{z}_{2}=-\left(a_{2 v}-j b_{2 v}\right) / a_{2 i}$, and $\underline{z}_{3}=0$, respectively. According to our assumption, the load is passive and therefore $\operatorname{Re}\left\{\underline{z}_{1}\right\}>0$ and $\operatorname{Re}\left\{\underline{z}_{2}\right\} \geq 0$, which further imply $a_{1 i} a_{1 v}<0$ and $a_{2 i} a_{2 v} \leq 0$, respectively.

Because current waveform (188) contains only cosine terms and voltage waveform is the same as in previous case 


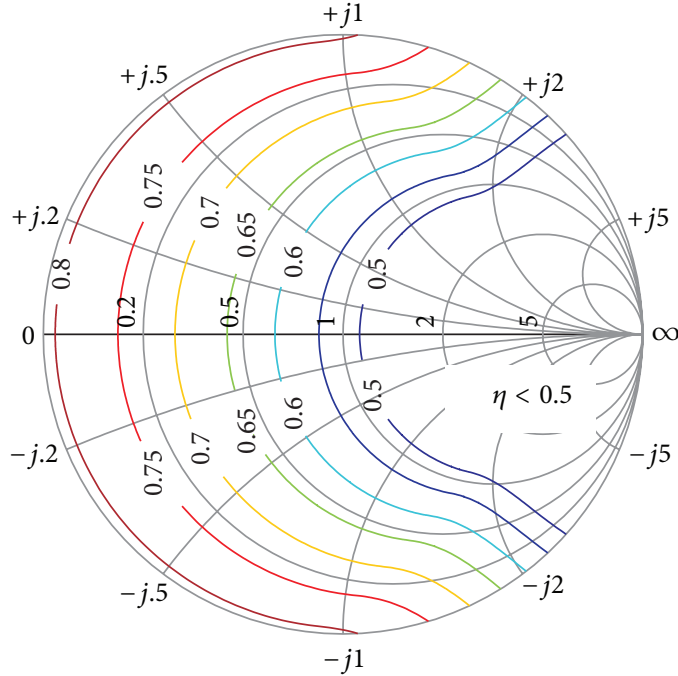

(a)

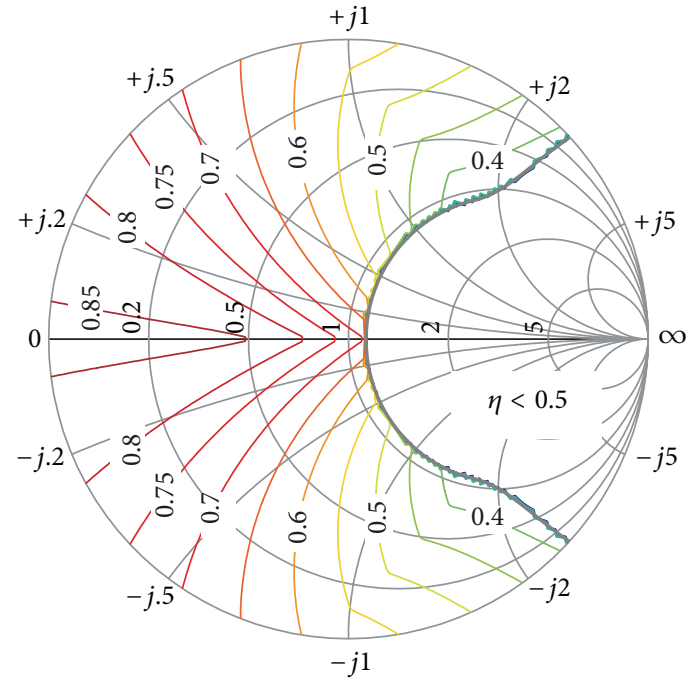

(b)

FIGURE 19: (a) Contours of maximal efficiency of PA and (b) contours of corresponding PUF, associated with the waveform pair (187)-(188) as functions of normalized second harmonic impedance $\underline{z}_{2 n}=\underline{z}_{2} / \operatorname{Re}\left\{\underline{z}_{1}\right\}$.

study, the procedure for calculation of maximal efficiency of PA with waveform pair (187)-(188) is the same as presented in Algorithm 32. In this case study the coefficients of fundamental and second harmonic of current waveform are $a_{1 i}=$ $(1+\sqrt{5}) / 2$ and $a_{2 i}=2 \sqrt{5} / 5$, respectively.

Maximal efficiency of PA associated with the waveform pair (187)-(188), as a function of normalized second harmonic impedance $\underline{z}_{2 n}=\underline{z}_{2} / \operatorname{Re}\left\{\underline{z}_{1}\right\}$, is presented in Figure 19(a). Efficiency of 0.8 is achieved at the edge of Smith chart, where second harmonic impedance has small resistive part. The theoretical upper bound $\eta=(1+\sqrt{5}) / 4 \approx 0.8090$ is attained when second harmonic is short-circuited. When this upper bound is reached, both second and third harmonic are short-circuited which implies that we are dealing with finite harmonic class-C $[6,8]$, or dually, when current and voltage interchange their roles, with finite harmonic inverse class-C $[6,9]$. Corresponding PUF, calculated according to (179), is presented in Figure 19(b). Peak value of PUF $\approx 0.8541$ is attained when second harmonic is short-circuited.

For example, for second harmonic impedance $\underline{z}_{2}=$ $0.07-j 0.4$ and current waveform (188), from Algorithm 32 it follows that $2 \lambda_{2 v} \leq 1-a_{2 v}$. Furthermore, according to step (iii) of Algorithm 32, maximal efficiency of PA is attained with voltage waveform of type (187) with coefficients $a_{2 v}=$ $-0.0626, b_{2 v}=-0.3578, a_{1 v}=-0.9374$, and $b_{1 v}=0.7155$ (see Figure 20). Corresponding efficiency, PUF, and normalized second harmonic impedance are $\eta=0.7584$, PUF $=0.6337$, and $\underline{z}_{2 n}=0.1208-j 0.6904$, respectively.

On the other hand, for $\underline{z}_{2}=0.05-j 0.7$ and current waveform (187) it follows that $2 \lambda_{2 v}>1-a_{2 v}$. Then, according to step (iii) of Algorithm 32, the maximal efficiency is attained with voltage waveform of type (187) with coefficients $a_{2 v}=$ $-0.0447, b_{2 v}=-0.6261, a_{1 v}=-0.9318$, and $b_{1 v}=1.0007$ (see Figure 21). Efficiency, PUF, and normalized second harmonic

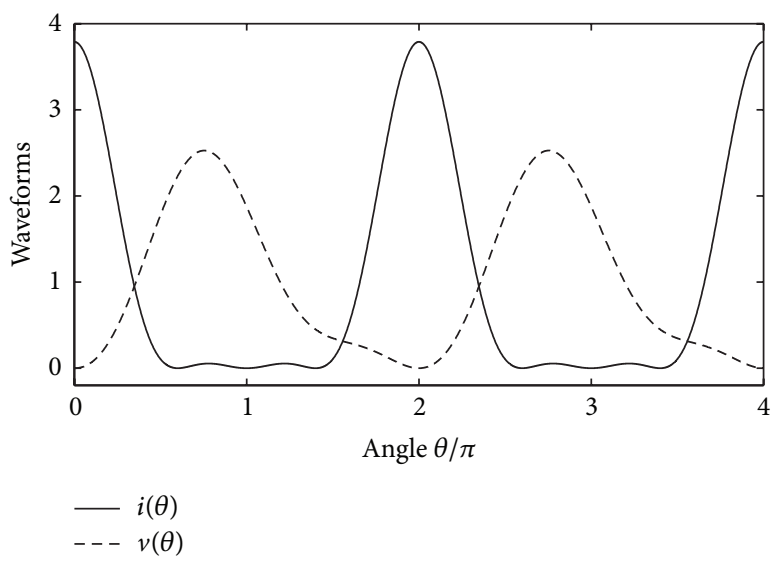

FIGURE 20: Waveform pair (187)-(188) that provides maximal efficiency for $\underline{z}_{2}=0.07-j 0.4$.

impedance are $\eta=0.7538, \mathrm{PUF}=0.5314$, and $\underline{z}_{2 n}=0.0868-$ $j 1.2156$, respectively.

7.2. Nonnegative Waveforms for $k=3$ in PA Efficiency Analysis. In this subsection we provide another two case studies of usage of description of nonnegative waveforms in PA efficiency analysis, this time with fundamental and third harmonic $(k=3)$.

Case Study 7.3. Let us consider current-voltage pair such that voltage is nonnegative waveform with fundamental and third harmonic:

$$
v(\theta)=1+a_{1 v} \cos \theta+b_{1 v} \sin \theta+a_{3 v} \cos 3 \theta+b_{3 v} \sin 3 \theta,
$$




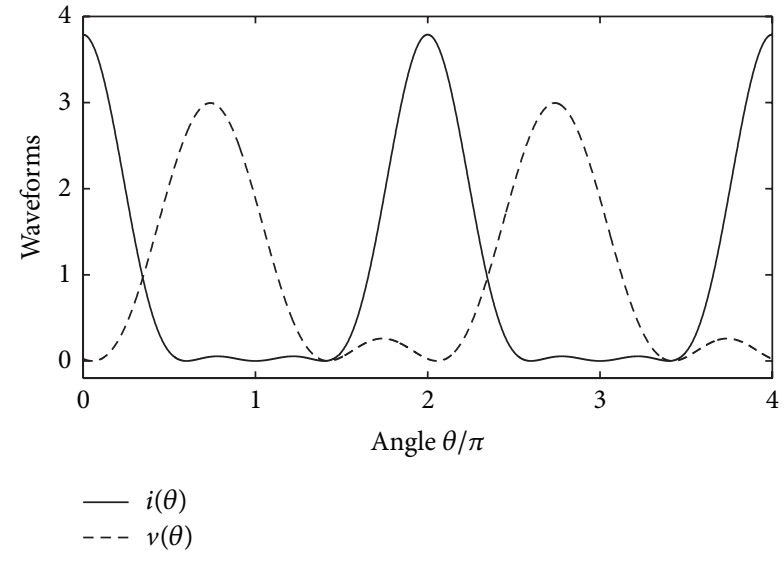

FIGURE 21: Waveform pair (187)-(188) that provides maximal efficiency for $\underline{z}_{2}=0.05-j 0.7$.

and current is nonnegative cosine waveform given by (188). Load impedances at fundamental, second, and third harmonic are $\underline{z}_{1}=-\left(a_{1 v}-j b_{1 v}\right) / a_{1 i}, \underline{z}_{2}=0$, and $\underline{z}_{3}=-\left(a_{3 v}-\right.$ $\left.j b_{3 v}\right) / a_{3 i}$, respectively. According to our assumption, the load is passive and therefore $\operatorname{Re}\left\{\underline{z}_{1}\right\}>0$ and $\operatorname{Re}\left\{\underline{z}_{3}\right\} \geq 0$, which further imply $a_{1 i} a_{1 v}<0$ and $a_{3 i} a_{3 v} \leq 0$.

In this subsection we consider the problem of finding maximal efficiency of PA with waveform pair (188)-(189) for given third harmonic impedance. As we mentioned earlier, problem of finding maximal efficiency of PA with current-voltage pair (188)-(189) for prescribed third harmonic impedance can be reduced to the problem of finding voltage waveform of type (189) with maximal coefficient $\left|a_{1 v}\right|$, for prescribed coefficients of third harmonic (see Section 5.2).

The following algorithm provides the procedure for calculation of maximal efficiency with current-voltage pair (188)-(189). The definition of function atan $2(y, x)$, which appears in step (iii) of the following algorithm, is given by (105).

Algorithm 33. (i) Choose $\underline{z}_{3}=r_{3}+j x_{3}$ such that $\left|\underline{z}_{3}\right| \leq 1 /\left|a_{3 i}\right|$,

(ii) calculate $a_{3 v}-j b_{3 v}=-\underline{z}_{3} a_{3 i}$ and $\lambda_{3 v}=\sqrt{a_{3 v}^{2}+b_{3 v}^{2}}$,

(iii) if $27 \lambda_{3 v}^{2} \leq\left(1-2 a_{3 v}\right)^{3}$ then calculate $a_{1 v}=-1-a_{3 v}$ and $b_{1 v}=-3 b_{3 v}$; else, calculate $\lambda_{1 v}=3\left(\sqrt[3]{\lambda_{3 v}}-\lambda_{3 v}\right), \theta_{0 v}-$ $\xi_{v} / 3=(1 / 3) \operatorname{atan} 2\left(b_{3 v}, a_{3 v}\right), a_{1 v}=-\lambda_{1 v} \cos \left(\theta_{0 v}-\xi_{v} / 3\right)$, and $b_{1 v}=-\lambda_{1 v} \sin \left(\theta_{0 v}-\xi_{v} / 3\right)$,

(iv) calculate efficiency $\eta=-a_{1 i} a_{1 v} / 2$,

(v) calculate $\underline{z}_{1}=-\left(a_{1 v}-j b_{1 v}\right) / a_{1 i}$ and $\underline{z}_{3 n}=\underline{z}_{3} / \operatorname{Re}\left\{\underline{z}_{1}\right\}$.

In this case study coefficients of fundamental and third harmonic of current waveform are $a_{1 i}=(1+\sqrt{5}) / 2$ and $a_{3 i}=(5-\sqrt{5}) / 10$, respectively. For the waveform pair (188)(189), maximal efficiency of PA as a function of normalized third harmonic impedance $\underline{z}_{3 n}=\underline{z}_{3} / \operatorname{Re}\left\{\underline{z}_{1}\right\}$ is presented in Figure 22. Efficiency of 0.8 is reached when third harmonic impedance has small resistive part. Peak efficiency $\eta=(1+$ $\sqrt{5}) / 4 \approx 0.8090$ is achieved when third harmonic is shortcircuited.

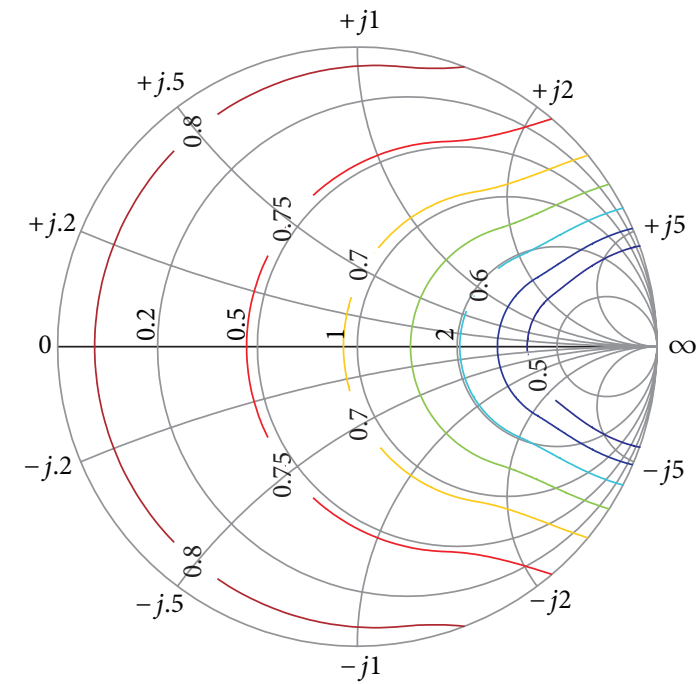

FIGURE 22: Contours of maximal efficiency of PA, associated with the waveform pair (188)-(189) as a function of normalized third harmonic impedance $\underline{z}_{3 n}=\underline{z}_{3} / \operatorname{Re}\left\{\underline{z}_{1}\right\}$.

For the present case study, in what follows we show that power utilization factor is proportional to efficiency. For voltage waveform of type (189) it is easy to see that $v(\theta+\pi)=$ $2-v(\theta)$ holds. This relation along with the fact that waveform $v(\theta)$ that provides maximal efficiency has at least one zero implies that $\max [v(\theta)]=2$. On the other hand, current waveform (188) is cosine waveform with positive coefficients and therefore $\max [i(\theta)]=i(0)=2+4 / \sqrt{5}$. Consequently, according to (179), the following relation holds:

$$
\operatorname{PUF}_{\text {Case study 7.3 }}=2(5-2 \sqrt{5}) \eta=1.0557 \eta \text {. }
$$

Clearly, the ratio $\mathrm{PUF} / \eta$ is constant and therefore in this case study PUF can be easily calculated from the corresponding efficiency. Accordingly, peak efficiency and peak value of PUF $_{\text {Case study 7.3 }}=3 \sqrt{5} / 2-5 / 2=0.8541$ are attained for the same voltage waveform (when third harmonic is shortcircuited).

In the first example, current waveform (188) and $\underline{z}_{3}=$ $0.2-j 0.5$ imply that $27 \lambda_{3 v}^{2} \leq\left(1-2 a_{3 v}\right)^{3}$. Then, according to Algorithm 33, the voltage waveform of type (189) that provides maximal efficiency has the following coefficients: $a_{3 v}=-0.0553, b_{3 v}=-0.1382, a_{1 v}=-0.9447$, and $b_{1 v}=$ 0.4146 (see Figure 23). Efficiency, PUF, and normalized third harmonic impedance are $\eta=0.7643$, PUF $=0.8069$, and $\underline{z}_{3 n}=0.3425-j 0.8564$, respectively.

In the second example, current waveform (188) and $\underline{z}_{3}=$ $0.1-j 1.1$ imply that $27 \lambda_{3 v}^{2}>\left(1-2 a_{3 v}\right)^{3}$. Then, according to Algorithm 33, the voltage waveform of type (189) that provides maximal efficiency has the following coefficients: $a_{3 v}=-0.0276, b_{3 v}=-0.3040, a_{1 v}=-0.9391$, and $b_{1 v}=$ 0.5807 (see Figure 24). Efficiency, PUF, and normalized third harmonic impedance are $\eta=0.7598$, PUF $=0.8021$, and $\underline{z}_{3 n}=0.1723-j 1.8952$, respectively. 


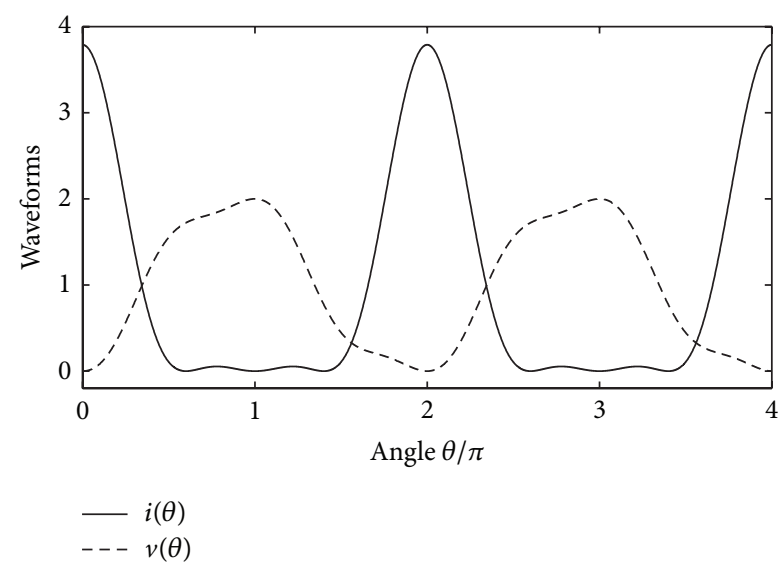

FIGURE 23: Waveform pair (188)-(189) that provides maximal efficiency for $\underline{z}_{3}=0.2-j 0.5$.

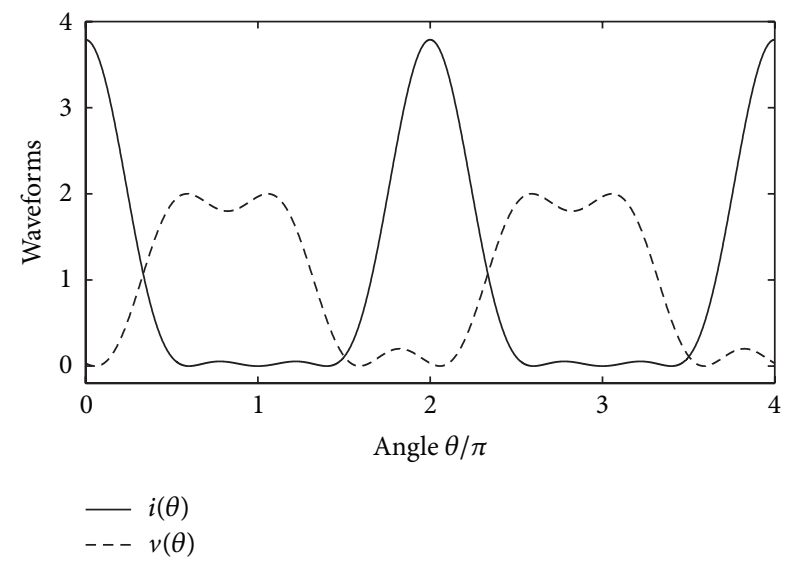

FIGURE 24: Waveform pair (188)-(189) that provides maximal efficiency for $\underline{z}_{3}=0.1-j 1.1$.

Case Study 7.4. In this case study let us consider currentvoltage pair, where current is normalized waveform of type (180) with conduction angle $\alpha=1.15 \pi\left(207^{\circ}\right)$ and voltage is nonnegative waveform of type (189). Substitution of $\alpha=$ $1.15 \pi$ and $I_{\mathrm{dc}}=1$ into (182) leads to $I_{D}=2.2535$. Furthermore, substitution of $\alpha=1.15 \pi$ and $I_{D}=2.2535$ into (180) leads to

$$
\begin{aligned}
& i(\theta) \\
& = \begin{cases}2.2535\left[\cos \theta-\cos \left(\frac{1.15 \pi}{2}\right)\right], & |\theta| \leq \frac{1.15 \pi}{2}, \\
0, & \frac{1.15 \pi}{2} \leq|\theta| \leq \pi .\end{cases}
\end{aligned}
$$

Similarly, substitution of $\alpha=1.15 \pi$ and $I_{D}=2.2535$ into (183) and (184) for $n=3$ yields coefficients of fundamental and third harmonic of waveform (191):

$$
a_{1 i}=1.4586, \quad a_{3 i}=-0.1026 .
$$

Because current waveform (191) contains only cosine terms and voltage waveform is the same as in previous case

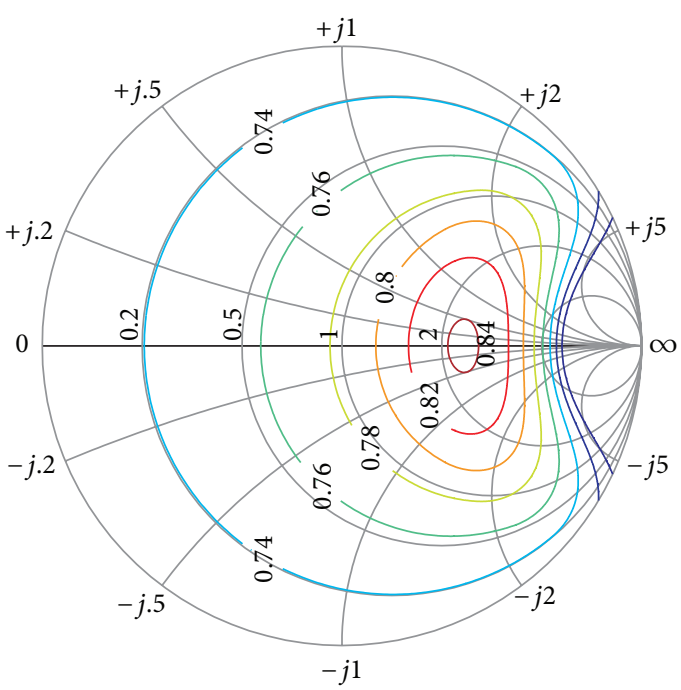

FIGURE 25: Contours of maximal efficiency of PA, associated with the waveform pair (189) and (191) as a function of normalized third harmonic impedance $\underline{z}_{3 n}=\underline{z}_{3} / \operatorname{Re}\left\{\underline{z}_{1}\right\}$.

study, the procedure for calculation of maximal efficiency of PA with waveform pair (189)-(191) is the same as that presented in Algorithm 33. In this case study the coefficients of fundamental and third harmonic of current waveform are given by (192).

For the waveform pair (189) and (191), maximal efficiency of PA as a function of normalized third harmonic impedance $\underline{z}_{3 n}=\underline{z}_{3} / \operatorname{Re}\left\{\underline{z}_{1}\right\}$ is presented in Figure 25. Efficiency of 0.84 is obtained in vicinity of $\underline{z}_{3 n}=2.3685$ (corresponding to $\underline{z}_{3}=$ 1.8750). Peak efficiency $\eta \approx 0.8421$ is achieved for voltage waveform of type (189) with coefficients $a_{1 v}=-2 / \sqrt{3}, a_{3 v}=$ $\sqrt{3} / 9$, and $b_{1 v}=b_{3 v}=0$.

In the course of finding power utilization factor, notice that current waveform of type (191) attains its maximum value for $\theta=0$. Insertion of $\max [i(\theta)]=i(0)=2.78$ and $\max [v(\theta)]=2$ for voltage waveform of type (189) into (179) leads to

$$
\mathrm{PUF}_{\text {Case study 7.4 }}=1.439 \eta \text {. }
$$

Again, the ratio PUF $/ \eta$ is constant and PUF can be easily calculated from the corresponding efficiency. Accordingly, peak value of $\mathrm{PUF}_{\text {Case study 7.4 }} \approx 1.2118$ and peak efficiency are attained for the same voltage waveform.

In the first example, current waveform (191) and $\underline{z}_{3}=$ $1-j 0.2$ imply that $27 \lambda_{3 v}^{2} \leq\left(1-2 a_{3 v}\right)^{3}$. Then, according to Algorithm 33, voltage waveform of type (189) which provides maximal efficiency has coefficients: $a_{3 v}=0.1026, b_{3 v}=$ 0.0205, $a_{1 v}=-1.1026$, and $b_{1 v}=-0.0616$ (see Figure 26). Efficiency, PUF, and normalized third harmonic impedance are $\eta=0.8042$, PUF $=1.1572$, and $\underline{z}_{3 n}=1.3228-j 0.2646$, respectively.

In second example, current waveform (191) and $\underline{z}_{3}=$ $1.5-j 1.2$ imply that $27 \lambda_{3 v}^{2}>\left(1-2 a_{3 v}\right)^{3}$. Then, according to Algorithm 33, voltage waveform of type (189) which provides maximal efficiency has coefficients: $a_{3 v}=0.1540, b_{3 v}=$ 


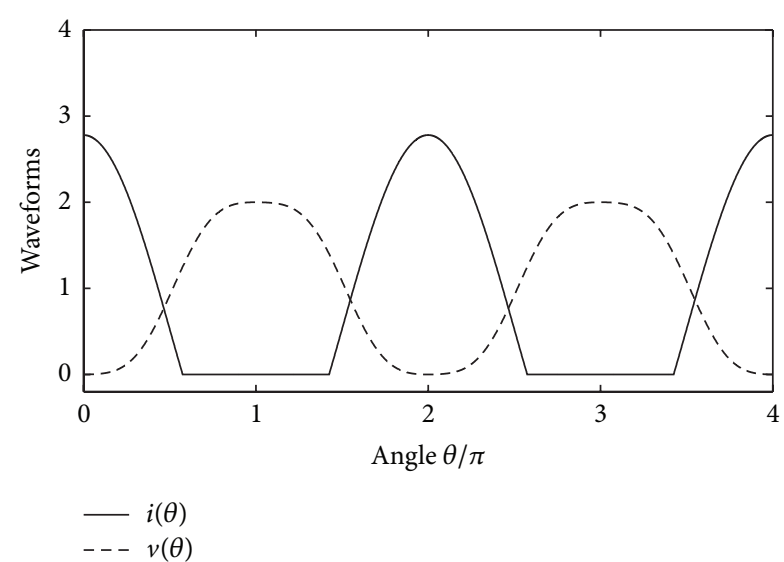

FIGURE 26: Waveform pair (189) and (191) that provides maximal efficiency for $\underline{z}_{3}=1-j 0.2$.

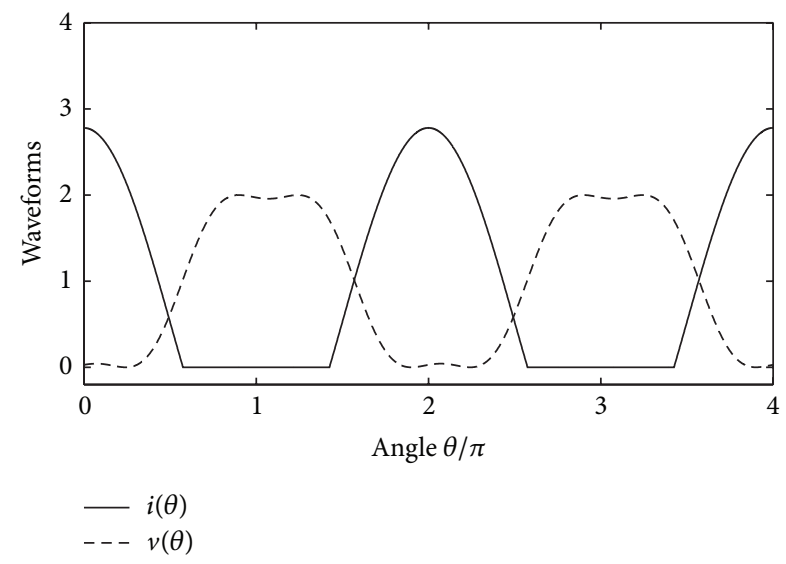

FIGURE 27: Waveform pair (189) and (191) that provides maximal efficiency for $\underline{z}_{3}=1.5-j 1.2$.

0.1232, $a_{1 v}=-1.1255$, and $b_{1 v}=-0.2575$ (see Figure 27). Efficiency, PUF, and normalized third harmonic impedance are $\eta=0.8208$, PUF $=1.1812$, and $\underline{z}_{3 n}=1.9439-j 1.5552$, respectively.

\section{Conclusion}

In this paper we consider a problem of finding general descriptions of various classes of nonnegative waveforms with fundamental and $k$ th harmonic. These classes include nonnegative waveforms with at least one zero, nonnegative waveforms with maximal amplitude of fundamental harmonic for prescribed amplitude of $k$ th harmonic, nonnegative waveforms with maximal coefficient of cosine part of fundamental harmonic for prescribed coefficients of $k$ th harmonic, and nonnegative cosine waveforms with at least one zero. Main results are stated in six propositions (Propositions 1, 6, 9, 18, 22, and 26), four corollaries (Corollaries 2-5), twenty remarks, and three algorithms. Four case studies of usage of closed form descriptions of nonnegative waveforms in PA efficiency analysis are considered in detail in Section 7.

\section{Appendices}

Here we provide a list of finite sums of trigonometric functions used in this paper (Appendix A) and brief account of the Chebyshev polynomials (Appendix B).

\section{A. List of Some Finite Sums of Trigonometric Functions}

Dirichlet kernel (e.g., see [27]) is as follows:

$$
D_{k-1}(\tau)=1+2 \sum_{n=1}^{k-1} \cos n \tau=\frac{\sin ((2 k-1) \tau / 2)}{\sin (\tau / 2)}
$$

Fejér kernel (e.g., see [27]) can be expressed in the following equivalent forms:

$$
\begin{aligned}
F_{k-1}(\tau) & =\frac{1}{k} \sum_{n=0}^{k-1} D_{n}(\tau)=1+\frac{2}{k} \sum_{n=1}^{k-1}(k-n) \cos n \tau \\
& =\frac{(1-\cos k \tau)}{k(1-\cos \tau)}
\end{aligned}
$$

Lagrange's trigonometric identity (e.g., see [28]) is as follows:

$$
S_{1}(\tau)=\sum_{n=1}^{k-1} \sin n \tau=\frac{\sin (k \tau / 2) \sin ((k-1) \tau / 2)}{\sin (\tau / 2)}
$$

In what follows we show that the following three trigonometric identities also hold:

$$
\begin{aligned}
& 2 \sum_{n=1}^{k-1}(k-n) \sin n \tau=\frac{k \sin \tau-\sin k \tau}{1-\cos \tau}, \\
& \sum_{n=1}^{k-1} \cos (k-2 n) \tau=\frac{\sin (k-1) \tau}{\sin \tau}, \\
& \sum_{n=1}^{k-1} n(k-n) \cos (k-2 n) \tau \\
& =\frac{\sin (k \tau) \cos \tau-k \cos (k \tau) \sin \tau}{2 \sin ^{3} \tau} .
\end{aligned}
$$

Denote $S_{2}(\tau)=2 \sum_{n=1}^{k-1}(k-n) \sin n \tau, S_{3}(\tau)=\sum_{n=1}^{k-1} \cos (k-$ $2 n) \tau$, and $S_{4}(\tau)=\sum_{n=1}^{k-1} n(k-n) \cos (k-2 n) \tau$.

Notice that $S_{2}(\tau)=2 k S_{1}(\tau)+d D_{k-1}(\tau) / d \tau$, which immediately leads to (A.4). 
Identity (A.5) can be obtained as follows:

$$
\begin{aligned}
\frac{\sin (k-1) \tau}{\sin \tau} & =\frac{e^{j(k-1) \tau}-e^{-j(k-1) \tau}}{e^{j \tau}-e^{-j \tau}} \\
& =e^{j k \tau} \frac{e^{-2 j \tau}-e^{-2 j(k-1) \tau}}{1-e^{-2 j \tau}} \\
& =e^{j k \tau} \sum_{n=1}^{k-1} e^{-2 j n \tau}=\sum_{n=1}^{k-1} e^{j(k-2 n) \tau} \\
& =\sum_{n=1}^{k-1} \cos (k-2 n) \tau
\end{aligned}
$$

From $4 n(k-n)=k^{2}-(k-2 n)^{2}$ it follows that $4 S_{4}(\tau)=$ $k^{2} S_{3}(\tau)+d^{2} S_{3}(\tau) / d \tau^{2}$, which leads to (A.6).

\section{B. The Chebyshev Polynomials}

The Chebyshev polynomials of the first kind $V_{n}(x)$ can be defined by the following relation (e.g., see [29]):

$$
V_{n}(x)=\cos n \tau, \quad \text { when } x=\cos \tau .
$$

The Chebyshev polynomials of the second kind $U_{n}(x)$ can be defined by the following relation (e.g., see [29]):

$$
U_{n}(x)=\frac{\sin (n+1) \tau}{\sin \tau}, \quad \text { when } x=\cos \tau .
$$

The Chebyshev polynomials satisfy the following recurrence relations (e.g., see [29]):

$$
\begin{gathered}
V_{0}(x)=1, \quad V_{1}(x)=x, \\
V_{n+1}(x)=2 x V_{n}(x)-V_{n-1}(x), \\
U_{0}(x)=1, \quad U_{1}(x)=2 x, \\
U_{n+1}(x)=2 x U_{n}(x)-U_{n-1}(x) .
\end{gathered}
$$

The first few Chebyshev polynomials of the first and second kind are $V_{2}(x)=2 x^{2}-1, V_{3}(x)=4 x^{3}-3 x, V_{4}(x)=8 x^{4}-$ $8 x^{2}+1, U_{2}(x)=4 x^{2}-1, U_{3}(x)=8 x^{3}-4 x$, and $U_{4}(x)=$ $16 x^{4}-12 x^{2}+1$.

\section{Conflict of Interests}

The authors declare that there is no conflict of interests regarding the publication of this paper.

\section{Acknowledgment}

This work is supported by the Serbian Ministry of Education, Science and Technology Development, as a part of Project TP32016.

\section{References}

[1] V. I. Arnol'd, V. S. Afrajmovich, Y. S. Il'yashenko, and L. P. Shil'nikov, Dynamical Systems V, Bifurcation Theory and Catastrophe Theory, Springer, Berlin, Germany, 1994.
[2] E. Polak, "On the mathematical foundations of nondifferentiable optimization in engineering design," SIAM Review, vol. 29, no. 1, pp. 21-89, 1987.

[3] N. S. Fuzik, "Biharmonic modes of a tuned RF power amplifier," Radiotehnika, vol. 25, no. 7, pp. 62-71, 1970 (Russian).

[4] P. Colantonio, F. Giannini, G. Leuzzi, and E. Limiti, "Class G approach for low-voltage, high-efficiency PA design," International Journal of RF and Microwave Computer-Aided Engineering, vol. 10, no. 6, pp. 366-378, 2000.

[5] F. H. Raab, "Maximum efficiency and output of class-F power amplifiers," IEEE Transactions on Microwave Theory and Techniques, vol. 49, no. 6, pp. 1162-1166, 2001.

[6] F. H. Raab, "Class-E, class-C, and class-F power amplifiers based upon a finite number of harmonics," IEEE Transactions on Microwave Theory and Techniques, vol. 49, no. 8, pp. 1462-1468, 2001.

[7] J. D. Rhodes, "Output universality in maximum efficiency linear power amplifiers," International Journal of Circuit Theory and Applications, vol. 31, no. 4, pp. 385-405, 2003.

[8] A. Juhas and L. A. Novak, "Comments on 'Class-E, class-C, and classF power amplifier based upon a finite number of harmonics," IEEE Transactions on Microwave Theory and Techniques, vol. 57, no. 6, pp. 1623-1625, 2009.

[9] M. Roberg and Z. Popović, "Analysis of high-efficiency power amplifiers with arbitrary output harmonic terminations," IEEE Transactions on Microwave Theory and Techniques, vol. 59, no. 8, pp. 2037-2048, 2011.

[10] A. Grebennikov, N. O. Sokal, and M. J. Franco, Switchmode RF Power Amplifiers, Elsevier/Academic Press, San Diego, Calif, USA, 2nd edition, 2012.

[11] T. Canning, P. J. Tasker, and S. C. Cripps, "Continuous mode power amplifier design using harmonic clipping contours: theory and practice," IEEE Transactions on Microwave Theory and Techniques, vol. 62, no. 1, pp. 100-110, 2014.

[12] A. Juhas and L. A. Novak, "General description of nonnegative waveforms up to second harmonic for power amplifier modelling," Mathematical Problems in Engineering, vol. 2014, Article ID 709762, 18 pages, 2014.

[13] V. I. Arnol'd, V. V. Goryunov, O. V. Lyashko, and V. A. Vasilev, Dynamical Systems VIII-Singularity Theory II, Applications, Springer, Berlin, Germany, 1993.

[14] D. Siersma, "Properties of conflict sets in the plane," Banach Center Publications, Polish Academy of Sciences, vol. 50, no. 1, pp. 267-276, 1999, Proceedings of the Banach Center Symposium on Geometry and Topology of Caustics (Caustics '98), Warsaw, Poland.

[15] M. van Manen, The geometry of conflict sets [Dissertation], Universiteit Utrecht, Utrecht, The Netherlands, 2003, http://igitur-archive.library.uu.nl/dissertations/2003-0912-123058/c4.pdf.

[16] Y. L. Sachkov, "Maxwell strata and symmetries in the problem of optimal rolling of a sphere over a plane," Sbornik: Mathematics, vol. 201, no. 7-8, pp. 1029-1051, 2010.

[17] I. A. Bogaevsky, "Perestroikas of shocks and singularities of minimum functions," Physica D: Nonlinear Phenomena, vol. 173, no. 1-2, pp. 1-28, 2002.

[18] Y. L. Sachkov, "Maxwell strata in the Euler elastic problem," Journal of Dynamical and Control Systems, vol. 14, no. 2, pp. 169234, 2008.

[19] M. Siino and T. Koike, "Topological classification of black holes: generic Maxwell set and crease set of a horizon," International Journal of Modern Physics, D: Gravitation, Astrophysics, Cosmology, vol. 20, no. 6, pp. 1095-1122, 2011. 
[20] F. H. Raab, "Class-F power amplifiers with maximally flat waveforms," IEEE Transactions on Microwave Theory and Techniques, vol. 45, no. 11, pp. 2007-2012, 1997.

[21] A. Juhas and L. A. Novak, "Maximally flat waveforms with finite number of harmonics in class- $F$ power amplifiers," Mathematical Problems in Engineering, vol. 2013, Article ID 169590, 9 pages, 2013.

[22] L. Fejér, "Über trigonometrische polynome," Journal für die Reine und Angewandte Mathematik, vol. 1916, no. 146, pp. 5382, 1916 (German).

[23] S. C. Cripps, "Bessel Waives [microwave bytes]," IEEE Microwave Magazine, vol. 10, no. 7, pp. 30-36, 117, 2009.

[24] L. N. Bryzgalova, "Singularities of the maximum of parametrically dependent function," Functional Analysis and Its Applications, vol. 11, no. 1, pp. 49-51, 1977.

[25] V. I. Arnold, A. A. Davydov, V. A. Vassiliev, and V. M. Zakalyukin, Mathematical Models of Catastrophes, Control of Catastrophic Process, Encyclopedia of Life Support Systems (EOLSS), EOLSS Publishers, Oxford, UK, 2006.

[26] S. C. Cripps, RF Power Amplifiers for Wireless Communications, Artech House, Norwood, Mass, USA, 2nd edition, 2006.

[27] A. Zygmund, Trigonometric Series, vol. 1, Cambridge University Press, Cambridge, UK, 2nd edition, 1959.

[28] A. Jeffrey and H. Dai, Handbook of Mathematical Formulas and Integrals, Elsevier/Academic Press, San Diego, Calif, USA, 4th edition, 2008.

[29] J. C. Mason and D. C. Handscomb, Chebyshev Polynomials, Chapman \& Hall, CRC Press, Boca Raton, Fla, USA, 2003. 


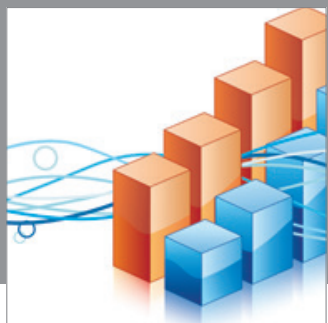

Advances in

Operations Research

mansans

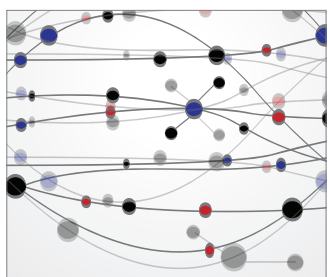

The Scientific World Journal
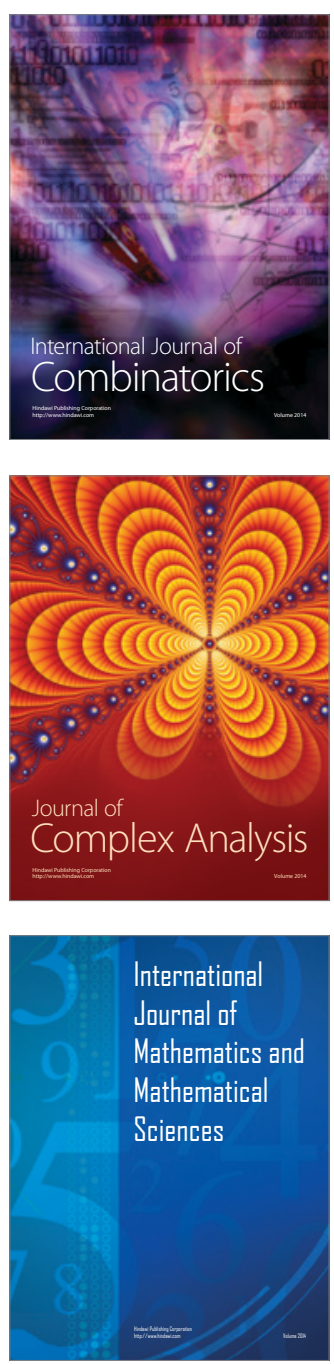
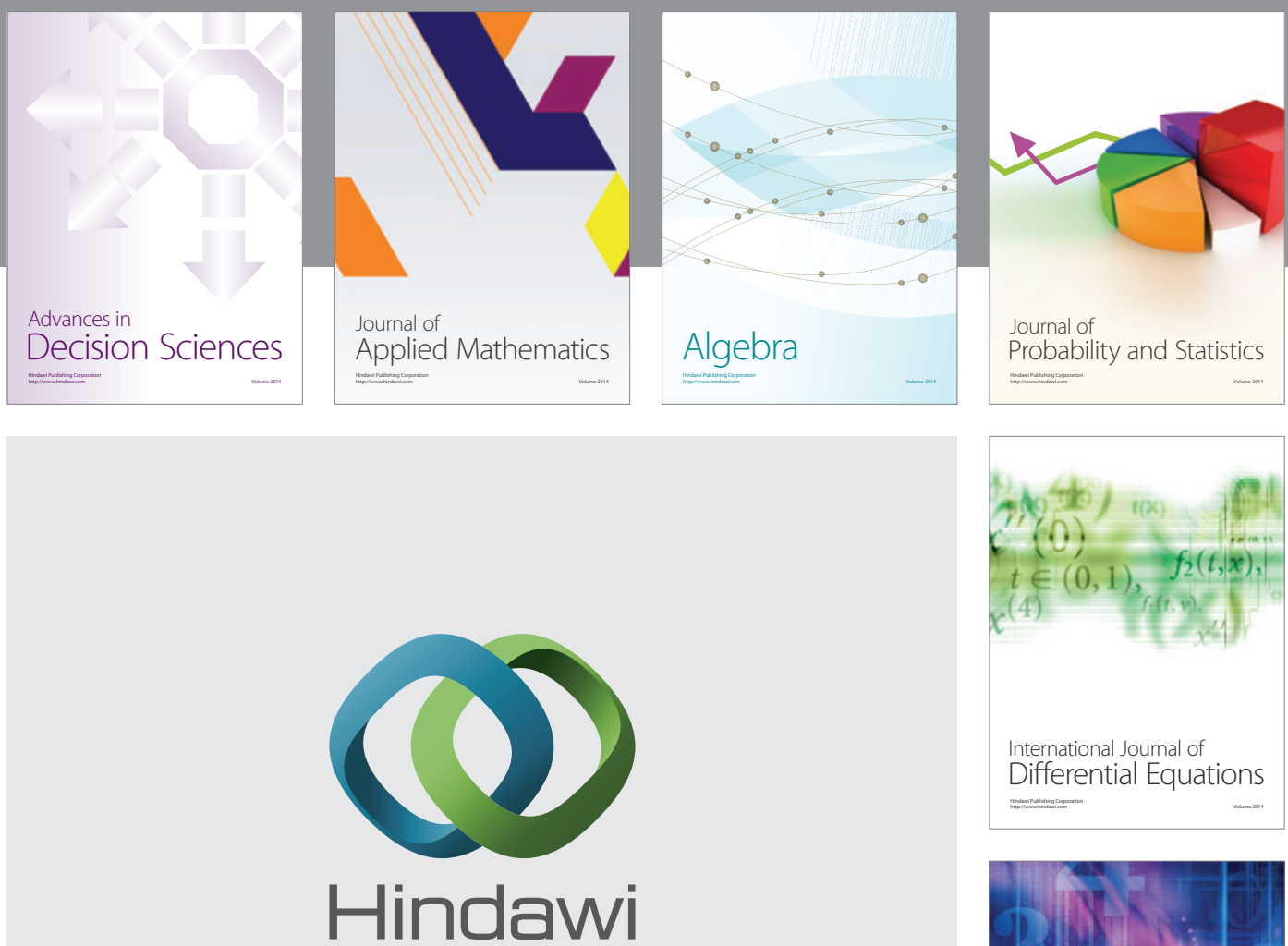

Submit your manuscripts at http://www.hindawi.com
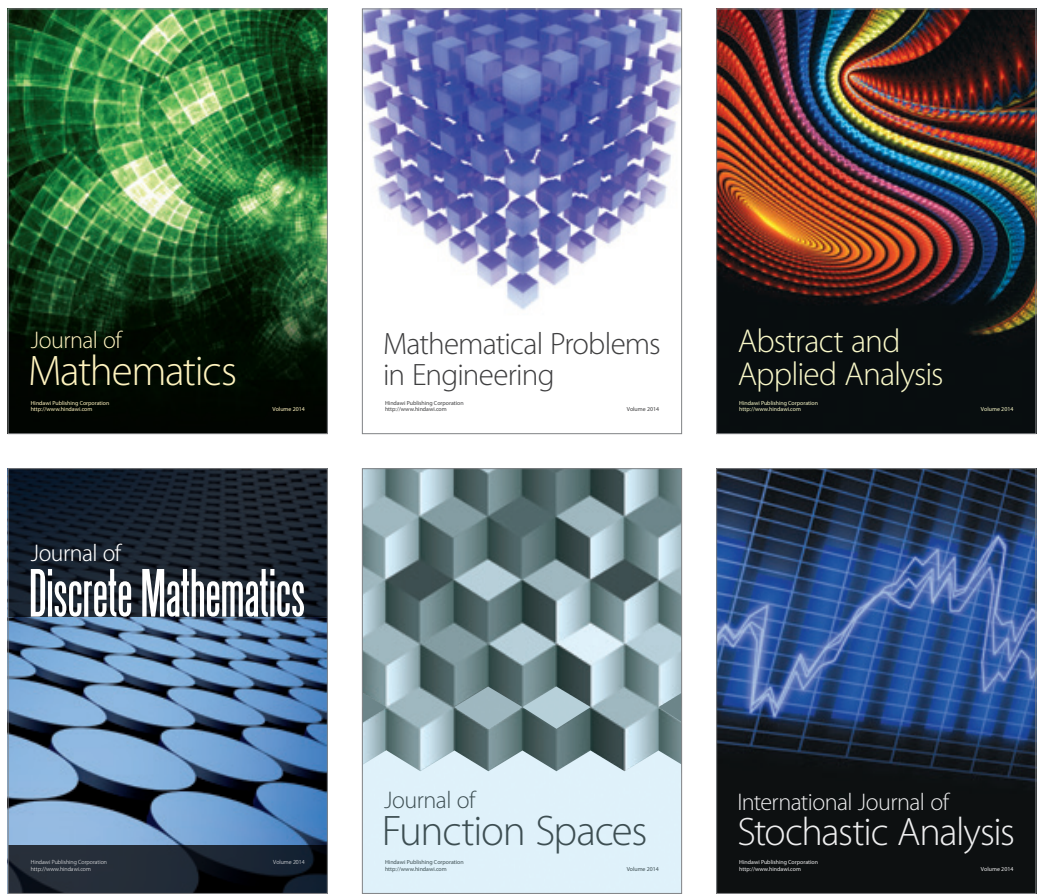

Journal of

Function Spaces

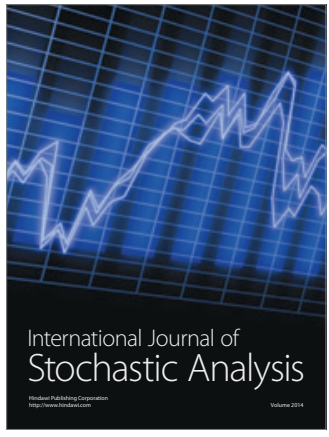

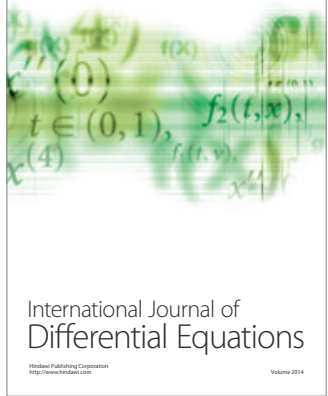
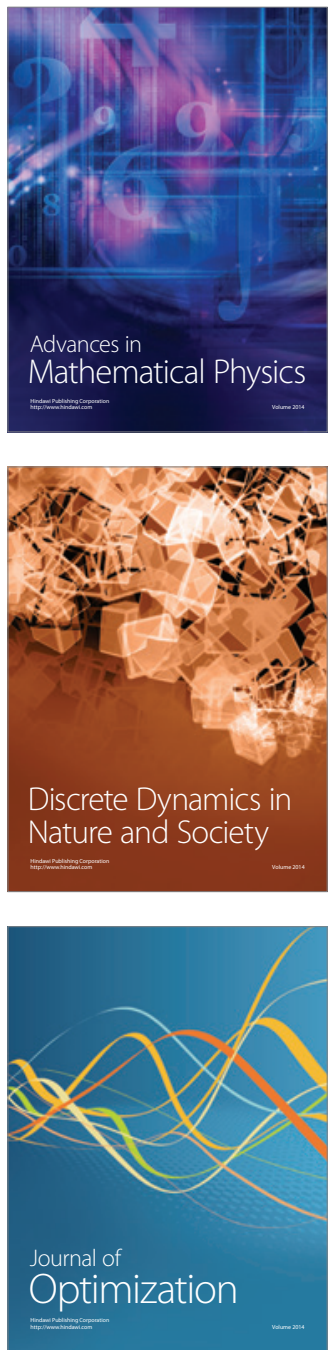